\title{
Un nouveau Toxodontidae (Mammalia, Notoungulata) du Pléistocène supérieur du Nordeste du Brésil
}

\author{
Claude GUÉRIN \\ Martine FAURE \\ UMR-CNRS 5276, Laboratoire de Géologie de Lyon, \\ Terre, Planètes, Environnement, \\ Université Claude Bernard-Lyon 1/ENS Lyon, \\ 27-43 boulevard du 11 novembre, F-69622 Villeurbanne cedex (France) \\ et Instituto Nacional de Arqueologia, \\ Paleontologia e Ambiento do Semi-Árido (INCT/CNPq), \\ Fundação Museu do Homem Americano (FUMDHAM), PI (Brésil) \\ claude.guerin@univ-lyon1.fr \\ martine.faure@mom.fr
}

MOTS CLÉS

taxonomie,

biométrie,

paléoécologie,

Amérique du Sud,

Piauí,

genre nouveau,

espèce nouvelle.
À Niède, en hommage à ses 25 ans de persévérance
sur les traces de la mégafaune dans les lagoas du sertão piauiense

Guérin C. \& Faure M. 2013. - Un nouveau Toxodontidae (Mammalia, Notoungulata) du Pléistocène supérieur du Nordeste du Brésil. Geodiversitas 35 (1): 155-205. http://dx.doi.org/10.5252/g2013n1a7

\section{RÉSUMÉ}

Les fossiles découverts par la Fundação Museu do Homem Americano dans la région du Parc National Serra da Capivara (SE du Piauí) nous ont permis de mettre en évidence deux espèces de Toxodontidae dans le Pléistocène supérieur du Nordeste du Brésil : Toxodon platensis Owen, 1837 et Piauhytherium capivarae n. gen., n. sp. Nous avons identifié T. platensis dans huit gisements nordestins, les principaux étant la Toca da Janela da Barra do Antonião, la Toca do Garrincho (tous deux sur la commune de São Raimundo Nonato, Piauí), la Lagoa dos Porcos à São Lourenço do Piauí, et Laje Grande à Pesqueira (Pernambouco). Il est représenté par une quarantaine de restes étudiables. Il est en moyenne un peu plus petit que la forme-type d'Argentine et du Sud du Brésil. Piauhytherium capivarae n. gen., n. sp. est présent dans neuf sites du Nordeste brésilien, les principaux étant la Toca do Garrincho, les Lagoas São Vitor, do Quari, et dos Porcos, tous de la région du Parc National Serra da Capivara. Il est représenté par plus de cent restes, parmi lesquels un crâne et une mandibule remarquablement bien conservés ; il est très différent de T. platensis par sa morphologie crânienne, et s'en distingue aussi par ses dents et son squelette post-crânien. Ses dents sont également différentes de celles de Trigodonops lopesi (Roxo, 1921) et Mixotoxodon larensis van Frank, 1957. À la Lagoa dos Porcos et à la Toca do Garrincho (Piauí) et à Fazenda Vista Alegre à Taperoá (Pernambouco) 
PALAVRAS CHAVES América do Sul, taxonomia, biometria, paleoecologia, Piauí, novo género, nova espécie.

\section{KEY WORDS}

South America, taxonomy, biometry, palaeoecology, Piauí, new genus, new species.
P. capivarae n. gen., n. sp. est sympatrique de T. platensis. Les rapports des segments de membres montrent que $P$. capivarae n. gen., n. sp., comme T. platensis, avait probablement des moeurs aquaphiles rappelant celles des hippopotames.

\section{RESUMO}

Um novo Toxodontidae (Mammalia, Notoungulata) do Pleistoceno superior do Nordeste do Brasil.

Os fósseis descobertos pela Fundação Museu do Homem Americano na região do Parque Nacional Serra da Capivara (Sudeste do Piauí) nos permitiu identificar duas espécies de Toxodontidae no Pleistoceno superior do Nordeste do Brasil, Toxodon platensis Owen, 1837 e Piauhytherium capivarae, n. gen., n. sp. Foi observado Toxodon platensis em oito sítios nordestinos, os principais são a Toca da Janela da Barra do Antonião, a Toca do Garrincho (os dois localizados no município de São Raimundo Nonato, Piauí), a Lagoa dos Porcos em São Lourenço do Piauí e Laje Grande em Pesqueira (PE). Está representado por uma quarentena de restos estudados. Ele tem, em média, uma forma um pouco menor que a forma - tipo da Argentina e do Sul do Brasil. Piauhytherium capivarae n. gen., n. sp. está presente em nove sítios do Nordeste brasileiro, os principais são a Toca do Garrincho, as Lagoas São Vitor, do Quari e dos Porcos, todos na regiáo do Parque Nacional Serra da Capivara. Foi identificado a partir de mais de uma centena de restos, entre os quais um crânio e uma mandíbula notadamente bem conservados; é muito diferente do T. platensis por sua morfologia craniana e, se distingue também, por seus dentes e esqueleto pós-craniano. Seus dentes são igualmente diferentes daqueles de Trigodonops lopesi e Mixotoxodon larensis. $\mathrm{Na}$ Lagoa dos Porcos, na Toca do Garrincho e Fazenda Vista Alegre em Taperoá (PB), P. capivarae n. gen., n. sp. é uma espécie simpátrica do T. platensis. As relaçóes dos segmentos dos membros mostram que P. capivarae n. gen., n. sp., como T. platensis teriam provavelmente hábitos aquáticos, lembrando aos dos hipopótamos.

\footnotetext{
ABSTRACT

A new Toxodontidae (Mammalia, Notoungulata) from the Upper Pleistocene of North Eastern Brazil.

Among the numerous fossils discovered by the Fundação Museu do Homem Americano in the Serra da Capivara National Park region (Piauí, Brazil), we were able to identify two species of Toxodontidae from the Upper Pleistocene of North Eastern Brazil, Toxodon platensis Owen, 1837 et Piauhytherium capivarae n. gen., n. sp. Toxodon platensis is present in eight Northeastern sites, the most important being the Toca da Barra do Antoniáo, the Toca do Garrincho, (all two in São Raimundo Nonato, Piauí), the Lagoa dos Porcos in São Lourenço do Piauí, and Laje Grande in Pesqueira (PE). Forty remains belong to it. These remains are generally a little smaller than the homologous pieces from the type form of Argentina and Southern Brazil. We found Piauhytherium capivarae n. gen., n. sp. in nine Northeastern sites, essentially the Toca do Garrincho and the Lagoas São Vitor, do Quari, and dos Porcos, all from the Serra da Capivara National Park region. More than one hundred remains belong to it, including a skull and a mandible very well preserved. These are quite different from those of T. platensis, and teeth and post-cranial skeleton are different too. The teeth are clearly distinguishable form those of Trigodonops lopesi (Roxo, 1921) et Mixotoxodon larensis van Frank, 1957. At the Lagoa dos Porcos, the Toca do Garrincho (Piauí) and Fazenda Vista Alegre in Taperoá (PB) P. capivarae n. gen., n. sp. is sympatric with $T$. platensis. The limb segments ratios show that $P$. capivarae n. gen., n. sp., like T. platensis, had an periaquatic hippopotamus way of life.
} 


\section{INTRODUCTION}

\section{HisTORIQUE DES DÉCOUVERTES}

La présence de Toxodontidae Owen, 1845 dans des gisements pléistocènes du Nordeste brésilien est connue depuis plus d'un demi-siècle (Paula Couto 1954, 1979, 1980 ; Vidal 1959 ; Rolim 1971, 1974a, b) ; en général ils avaient alors été attribués au genre et à l'espèce les plus connus de cette famille, Toxodon platensis Owen, 1837, identifié dans le Sud du pays dès le début des recherches paléontologiques en Amérique du Sud. Plus récemment, divers auteurs ont signalé ponctuellement des restes de Toxodon Owen, 1837 (ou de Toxodontidae indét.) dans des sites du Rio Grande do Norte, du Sergipe, du Bahia, du Pernambuco (entre autres Porpino et al. 2004 ; Dantas et al. 2005 ; Dantas \& Tasso 2007; Silva Alves \& Barreto 2007; Oliveira et al. 2009; Ribeiro \& Carvalho 2009; Silva et al. 2010). Dernièrement encore au Pernambuco, nous avons mis en évidence la présence de Toxodontidae dans la Lagoa do Urí de Cima à Salgueiro. Depuis 2010, cette lagoa fait l'objet de travaux par une équipe de fouille de la FUMDHAM et de l'INAPAS, dans le cadre du Projeto de Integração do Rio São Francisco com as Badas Hidrográficas do NE Setentrional-PISF, sous la responsabilité d'Anne-Marie Pessis. Ces travaux sont en cours et dans l'immédiat, seuls des petits fragments dentaires ont été recueillis, ne permettant pas de détermination spécifique.

Quelques rares auteurs (Rolim 1974a, b ; Cartelle 1994) ont envisagé l'existence dans la région d'un deuxième toxodontidé, Trigodonops lopesi (Roxo, 1921). Dans le cadre d'un mémoire de Mestrado, Mendonça (2007) a tenté de faire une révision des Toxodontidae du Brésil fondée sur l'étude des restes crâniens et dentaires. Il considère qu'au moins deux espèces distinctes de Toxodontidae ont coexisté dans le Pléistocène du Nordeste du Brésil Toxodon platensis et Toxodon lopesi (réattribuant ainsi T. lopesi au genre Toxodon), et peut-être Mixotoxodon larensis van Frank, 1957 qu'il estime limité à l'extrême Nord du Brésil. Nous attendons que ceci soit publié, car un extrait très partiel de ce mémoire (limité à son introduction et à sa conclusion) diffusé en ligne par l'USP ne permet pas d'en juger.
Grâce aux recherches menées par la Fundação Museu do Homem Americano, sous l'impulsion de Niède Guidon, des restes de Toxodontidae ont été découverts à diverses reprises au sud-est de l'État du Piauí, dans la région de São Raimundo Nonato, (entre $42.4^{\circ}$ et $42.7^{\circ} \mathrm{W}$ et $8.8^{\circ}$ à $9.3^{\circ} \mathrm{S}$ ) (Guérin et al. 1993a, b, 1996a ; Guérin \& Faure 2008). Dès 1991, nous avions signalé la présence d'un Toxodontidae (Guérin 1991), mais ne disposions pas d'assez de matériel pour le déterminer avec précision. Les riches gisements de la Lagoa do Quari (Parenti et al. 2003 ; Chaves et al. 2008) et surtout de la Lagoa dos Porcos, fouillée en 2010, ont livré de nouveaux fossiles qui, s'ajoutant à ceux déjà mis au jour dans trois autres sites de la région du Parc national Serra da Capivara (la Toca da Janela da Barra do Antonião, la Toca do Garrincho et la Lagoa São Vitor) ainsi qu'à la Lagoa da Pedra à Conceição das Creoulas (Salgueiro, Pernambuco) (Guérin 1993 ; Guérin et al. 1996b), permettent désormais la détermination générique et spécifique des Toxodontidae de la région, et la mise en évidence d'un genre nouveau et d'une espèce nouvelle.

\section{LES GENRES DE TOXODONTIDAE DANS LE} QuATERNAIRE DU BRÉSIL SEPTENTRIONAL

Au Pliocène et au Quaternaire, la famille des Toxodontidae se caractérise par sa très grande biodiversité, qui a engendré une énorme confusion taxonomique. Répartis dans deux ou trois sous-familles dont les noms et les définitions varient selon les auteurs, ce sont en effet près de 25 genres, tous sud-américains, qui ont quelque chance d'être valides (van Frank 1957 ; Lavocat 1958 ; Pascual 1965 ; Pascual et al. 1966 ; Herbert \& Alvarez 1972 ; Paula Couto 1979, 1981, 1982 ; Bond et al. 1995 ; McKenna \& Bell 1997 ; Saint-André 1999). Quatre de ces genres tout au plus (Mixotoxodon van Frank, 1957 ; Abothrodon Paula Couto, 1944 ; Trigodonops L. Kraglievich, 1930 ; Toxodon) pourraient, selon les auteurs, être présents dans le Pléistocène supérieur du Brésil septentrional (Lavocat 1958 ; Paula Couto 1979). Marshall et al. (1984), qui ne parlent pas de Mixotoxodon mais citent Trigodonops et Abothrodon dans le Pléistocène d'Amazonie, envisagent la présence possible de Trigodonops dans le Quaternaire du Nordeste brésilien. Cartelle (1994), qui ne cite pas non 
plus Mixotoxodon ni d'ailleurs Abothrodon, considère pour sa part que dans le Pléistocène supérieur du Nord-Est du Brésil ont cohabité Toxodon, au muffle large, d'origine méridionale mais émigré vers le Nord à la suite d'un refroidissement, et Trigodonops lopesi, au muffle étroit, d'origine intertropicale.

Mixotoxodon est défini dans le Pléistocène du Vénézuéla et attesté en Amérique centrale au Costa Rica, Honduras, Salvador et au Nicaragua. L'espèce-type, et unique, est $M$. larensis. On connait la mandibule avec ses dents, ainsi que des dents supérieures et inférieures isolées et quelques os du squelette postcrânien. En 1959 de Porta a défini une nouvelle sous-espèce de Colombie, $M$. larensis crusafonti de Porta, 1959, d'après un fragment de mandibule portant $\mathrm{P} / 4-\mathrm{M} / 2$ et l'alvéole de $\mathrm{M} / 3$.

Abothrodon a pour espèce-type $A$. pricei Paula Couto, 1944, fondée sur une molaire inférieure gauche (probablement une M/2) de Cruzeiro do Sul (Haut Rio Juruá, Acre, Brésil). L'âge est incertain. Neotrigodon Spillmann, 1949, des rives du Rio Utoquinea au Pérou, en est probablement synonyme (Paula Couto 1979), la grande simplification des molaires supérieures du second correspondant bien à celle des molaires inférieures du premier.

Trigodonops est monospécifique avec T. lopesi, présumé du Pliocène du Haut Rio Juruá (Acre). Toxodon lopesi fut originellement attribué à Toxodon. La diagnose originelle de l'espèce est diffuse dans l'article de Roxo (1921). Le genre Trigodonops a été défini incidemment à l'occasion d'une analyse bibliographique, et sa description a été reprise par son auteur l'année suivante (Kraglievich 1930, 1931). Les diagnoses du genre et de l'espèce sont identiques. L'holotype, qui n’a pas de provenance géographique ni stratigraphique précise, est un fragment de mandibule gauche portant $\mathrm{P} / 3-\mathrm{M} / 2$, conservé au Departamento Nacional da Produção Mineral à Rio de Janeiro. Paula Couto (1982: 19) signale l'espèce dans au moins deux autres sites sur la rive gauche du Rio Juruá, dans un niveau datant probablement du Pléistocène final ; ce nouveau matériel se limite à quelques dents. Ferrusquia-Villafranca (1978) attribuait avec beaucoup de doutes à Trigodonops sp. un fossile du Pliocène de Peńata (Colombie). Rolim (1974b) semble avoir été le premier à attribuer au genre certains fossiles du Nordeste brésilien.
Toxodon est, de loin, le genre de Toxodontidé le plus connu. Il est répandu dans le Quaternaire de l'Amérique du Sud, notamment en Argentine, Uruguay, Paraguay, Bolivie, Brésil et Vénézuéla ; il semble qu'il n'ait jamais été signalé au dessus de 2000 mètres d'altitude (Hoffstetter 1986) et est inconnu à l'Ouest des Andes, au Chili, au Pérou et en Équateur. Il aurait existé en Amérique centrale jusqu'au Nicaragua du Nord. Ferrusquia-Villafranca (1978) parlait d'un Toxodon sp. dans le Pléistocène moyen récent de La Coca (Panama) et indiquait que le genre a été trouvé au Salvador, et peut-être au Guatemala ; mais Cisneros (2005) estime que le Toxodontidae de la Coca à Panama est probablement un Mixotoxodon. Si Toxodon platensis est l'espèce la mieux connue et la plus fréquemment mentionnée, de nombreuses autres attribuées au même genre ont été décrites en Argentine, dont au moins quatre ou cinq pourraient être valides: - Toxodon chapadmalalensis Ameghino, 1908, du Chapadmalalien (Pliocène), pour laquelle un néotype a été désigné, semble le premier représentant du genre ; sa taille est inférieure à celle des espèces pampéennes ; on lui a attribué un fémur de Miramar, dont la position stratigraphique est douteuse, et dans lequel est fichée une pointe de projectile en quartzite (Bond et al. 1995) ;

- Toxodon darwini Burmeister, 1866 et T. ensenadensis Ameghino, 1887, plus grand, sont tous deux de l'Ensénadien (Pléistocène inférieur). Herbst \& Alvarez (1972), qui n'ont pas retrouvé l'holotype du second, s'interrogeaient sur la validité de ces espèces et considéraient qu'une révision de tout le matériel s'imposait ;

- Toxodon gracilis Gervais \& F. Ameghino, 1880, du Bonaérien et du Lujánien (Pléistocène moyen et supérieur), est pour Miño Boilini et al. (2006) une espèce valide, significativement plus petite que les autres ;

- Toxodon gezi C. Ameghino, 1917, de taille relativement petite, est de la rive gauche du Rio Paraná, dans la Formation Yupoi (Ensénadien/Lujánien) de la province de Corrientes. L'ensemble des dents a été décrit par Herbst \& Alvarez (1972). Toxodon aguirrei Ameghino, 1917 en est le principal synonyme. Pour Miño Boilini \& Bond (2004) et Miño Boilini et al. (2006), T. gezi et T. aguirrei sont des synonymes ré- 
cents de T. platensis. En fait, seul Toxodon, et surtout T. platensis, est bien connu, grâce aux découvertes faites en Argentine, même si les descriptions de ses éléments anatomiques post-crâniens sont plutôt rares dans la littérature scientifique.

Nous n'avons pas trouvé trace de Mixotoxodon, de Trigodonops ni d'Abothrodon parmi le matériel brésilien que nous avons étudié. Nous n'avons reconnu que deux genres comptant chacun une espèce, Toxodon platensis et Piauhytherium capivarae n. gen., n. sp. que nous allons décrire ci-après.

$\begin{array}{ll}\text { ABRÉVIATIONS } \\ \text { abs. } & \text { absolu ; } \\ \text { anat. } & \text { anatomique ; } \\ \text { art. } & \text { articulaire ; } \\ \text { D } & \text { molaire de lait ; } \\ \text { DAP } & \text { diamètre antéro-postérieur ; } \\ \text { DDV } & \text { diamètre dorso-ventral ; } \\ \text { dia. } & \text { diaphyse ; } \\ \text { DT } & \text { diamètre transversal ; } \\ \text { dist. } & \text { distal ; } \\ \text { H } & \text { hauteur ; } \\ \text { H ant. } & \text { hauteur antérieure ; } \\ \text { L } & \text { longueur ; } \\ \text { l } & \text { largeur ; } \\ \text { min. } & \text { minimum ; } \\ \text { M } & \text { molaire ; } \\ \text { Mc } & \text { métacarpien ; } \\ \text { Mt } & \text { métatarsien ; } \\ \text { n } & \text { nombre de spécimens ; } \\ \text { P } & \text { prémolaire ; } \\ \text { prox. } & \text { proximal. }\end{array}$

Le rang de chaque dent est indiqué sous forme fractionnaire, en numérateur pour une dent supérieure et en dénominateur pour une inférieure.

L'indice d'hypsodontie est exprimé par le rapport $100 \times \mathrm{H} / \mathrm{L}$.

\section{MATÉRIEL ET MÉTHODES}

Nous avons pu comparer notre matériel aux restes pléistocènes argentins (pièces originales et moulages) et brésiliens conservés dans diverses grandes Collections de paléontologie (Zoologisk Museum à Copenhague, Muséum national d'Histoire naturelle à Paris, Natural History Museum à Londres, Muséum d'Histoire naturelle de Genève, Museu nacional de Rio de Janeiro). Nous avons aussi consulté des
Collections paléontologiques du Nordeste brésilien, notamment du Museu Câmara Cascudo de Natal (Rio Grande do Norte), de l'Université fédérale du Pernambuco à Recife (UFPE), du Cabinet d'Histoire naturelle du Gimnasio pernambucano à Recife et du Museu diocesano Dom José de Sobral (Ceará).

Tous les fossiles de la région du Parc National Serra da Capivara, notamment ceux figurés dans la présente étude, sont conservés dans les Collections de la Fundação Museu do Homem Americano (FUMDHAM), au Centro Cultural Sérgio Motta, à São Raimundo Nonato, Piauí.

Pour pouvoir comparer à l'aide des diagrammes des rapports (diagrammes «de Simpson») les proportions relatives des os des membres des diverses espèces, il nous fallait un groupe externe de référence. Faute d'autres fossiles appartenant à l'ordre des Notoungulata, nous avons choisi une famille d'Ongulés de l'Ancien Monde répondant à des exigences d'anatomie fonctionnelle voisines de celles qu'ont connu les Toxodontidés du Pléistocène supérieur : masse corporelle très élevée, mésaxonie avec tridactylie, graviportalité. Au sein de la famille des Rhinocerotidae, qui répond à ces conditions, nous disposons d'un très important échantillon de l'espèce actuelle Diceros bicornis (Linnaeus, 1758) comportant les squelettes post-crâniens de 30 à 40 individus adultes (valeurs in Guérin [1980], auxquelles s'ajoutent quelques spécimens nouveaux) et nous avons donc utilisé ses valeurs moyennes comme référentiel.

Toutes les dimensions sont en millimètres.

\section{SYSTÉMATIQUE}

Ordre NOTOUNGULATA Roth, 1903

Famille TOXODONTIDAE Owen, 1845

Genre Piauhytherium n. gen.

ESPÈCE TYPE. - Piauhytherium capivarae n. sp.

DiAgNoSE. - Le genre étant monospécifique, la diagnose est commune au genre et à l'espèce (voir ci-après).

ÉTYMologie. - De Piauhy, nom de l'ancienne "Capitania do Piauhy ", qui accéda à l'indépendance en 1759 et deviendra en 1889 l'État fédéré brésilien du Piauí. 


\section{Piaubytherium capivarae n. sp.}

Toxodon sp. - Guérin 1991: 570 partim. - Guérin et al. 1993a: 309, tableau 1, 323 partim ; 1993b: 194 partim ; 1996a: 63 tableau 1, 78 partim. — Guidon et al. 1993: 488, 489 partim. - Parenti et al. 1996: 346 partim. Peyre et al. 1998: 357 partim.

Toxodontidé de genre indét. - Guérin et al. 1996b: 341.

Mixotoxodon sp. - Parenti et al. 1996: 346 partim.

Trigodonops sp. - Parenti et al. 1996: 346 partim.

cf. Trigodonops-Guérin \& Faure 2008: 84, tableau.

HolotyPE. - Crâne complet n ${ }^{\circ} 188265$ de la Lagoa dos Porcos à São Lourenço do Piauí (Figs 1-4).

ÉTYMOlogie. — De la «Serra da Capivara» (= Montagne du Capivara), capivara étant le nom brésilien du grand rongeur Hydrochoerus hydrochaeris (Linnaeus, 1766).

MATÉRIEL RAPPORTÉ. - Ce nouveau binôme est représenté par plus de cent restes, nous l'avons identifié dans neuf sites du Nordeste Brésilien, principalement la Toca do Garrincho, les Lagoas São Vitor, do Quari, et dos Porcos, tous de la région du Parc National Serra da Capivara.

- Crâne sub-complet $n^{\circ} 185391$ et crâne incomplet du côté droit et à l'arrière $\mathrm{n}^{\circ} 185465$, tous deux de la Lagoa dos Porcos ; crâne incomplet mais non déformé de la Lagoa São Vitor n ${ }^{\circ} 48947$, associé à quatre dents jugales n 116393 ; crâne écrasé n ${ }^{\circ} 108998$ de la Lagoa do Quari. - Mandibule sub-complète de la Lagoa dos Porcos $\mathrm{n}^{\circ} 187141$; hémimandibule droite incomplète de la Lagoa São Vitor portant P/4-M/3, n 48061.

- Dents antérieures: quatre incisives du crâne $n^{\circ} 185391$, deux I/ 1 et une I/2 de la mandibule $\mathrm{n}^{\circ} 187141$ de la Lagoa dos Porcos, et deux défenses isolées du même site, I2/ $\mathrm{n}^{\circ} 184209$ et I/3 185991 ; une I supérieure (?) $n^{\circ} 37024-2$; une $I / 2 n^{\circ} 37001$ et deux I/3 n' 35844, et 35975 , toutes de la Toca do Garrincho.

- Dents jugales supérieures: quatre rangées dentaires correspondant au crâne $n^{\circ} 188265$ et $n^{\circ} 185391$ de la Lagoa dos Porcos, et 11 jugales supérieures isolées du même site, P2/ n ${ }^{\circ} 184262,185337,187118, \mathrm{P} 3 /$ $\mathrm{n}^{\circ} 184622,184982,187183, \mathrm{M} 1 / \mathrm{n}^{\circ} 183770,184607$, $\mathrm{M} 2 / \mathrm{n}^{\circ} 184244,184403,184510$; une prémolaire et trois molaires de la Lagoa São Vitor n ${ }^{\circ} 116393$ (associées au crâne $n^{\circ}$ 48947) ; une rangée incomplète P4/-M3/ de la Lagoa do Quari (avec crâne n $\left.{ }^{\circ} 108998\right)$; quatre prémolaires isolées de la Lagoa do Quari ( $\mathrm{n}^{\circ} 108716,108693$, 108664-2 et 108787); quatre prémolaires ( $\mathrm{n}^{\circ} 35879-1$, $35951,35921-1$ et 35898) et trois molaires ( ${ }^{\circ} 35898$, 35926 et 35899-2) isolées de la Toca do Garrincho. - Dents jugales inférieures: deux rangées dentaires de la mandibule ${ }^{\circ} 187141$ et la rangée dentaire P/3-M/3 $\mathrm{n}^{\circ} 186943$ de la Lagoa dos Porcos, et 9 jugales isolées du même site, $\mathrm{P} / 2 \mathrm{n}^{\circ} 184510, \mathrm{P} / 3 \mathrm{n}^{\circ} 188315, \mathrm{P} / 4$ $\mathrm{n}^{\circ} 186936$, trois $\mathrm{M} / 1 \mathrm{n}^{\circ} 184247,184443,185639$, $\mathrm{M} / 2 \mathrm{n}^{\circ} 186902$, deux M/3 n 184956 et 187151 ; une rangée $\mathrm{P} / 4-\mathrm{M} / 3$ de la Lagoa São Vitor (sur l'hémimandibule $\mathrm{n}^{\circ} 48061$, Fig. 10) ; une prémolaire et une molaire isolées de la Toca do Garrincho $\left(\mathrm{n}^{\circ} 35889-1\right.$ et 35987) ; une molaire isolée de la Lagoa do Quari ( $\left.{ }^{\circ} 108980\right)$.

- Humérus: deux fragments distaux n ${ }^{\circ} 181717$ et 185924 de la Lagoa dos Porcos ; trois (dont deux subcomplets) $\mathrm{n}^{\circ} 108963,108981$ et 108884 de la Lagoa do Quari ; un incomplet de Sobral (Ceará).

- Radius complet $n^{\circ} 184373$ de la Lagoa dos Porcos. - Cubitus complet no 184229 de la Lagoa dos Porcos. - Mc II : un de la Lagoa da Pedra à Conceição das Creoulas (PE) $n^{\circ}$ LP 43-5.

- Mc III : un gauche complet de la Lagoa do Quari $\mathrm{n}^{\circ} 109005$ (Fig. 11), un incomplet de la Lagoa São Vitor $\mathrm{n}^{\circ} 48809$.

- Mc IV: un droit $n^{\circ} 187847$ et un gauche complet $n^{\circ} 188050$ (Fig. 13) de la Lagoa dos Porcos; un de la Lagoa do Santo à Currais Novo et un de la Fazenda Vista Alegre à Taperoá (RN).

- Fémur: huit dont six complets de la Lagoa dos Porcos, $n^{\circ} 184388,185449,185451,185662,185803$, 185985,187103 et 187805 ; six dont quatre complets de la Lagoa do Quari, n 108878 (Fig. 14), n 108872, 108974, 108992, 109056, 109059; deux de la Lagoa da Cruz à Nova Cruz (RN).

- Tibia-péroné: un complet droit de la Lagoa dos Porcos, $n^{\circ} 187920$; un tibia sub-complet abîmé et écrasé $\mathrm{n}^{\circ} 108967$ et un fragment distal de tibia $\mathrm{n}^{\circ} 79644-6$ de la Lagoa do Quari.

- Calcanéum : un incomplet roulé de la Lagoa do Quari $n^{\circ} 79803-8$; un incomplet de la Lagoa do São Vitor $\mathrm{n}^{\circ} 48912$.

Niveau type et localité type. — Pléistocène supérieur, Lagoa dos Porcos, (site n ${ }^{\circ} 1302$ de l'inventaire de la FUMDHAM), municipio de Sáo Lourenço do Piauí, dans la région du Parc national Serra da Capivara, au SE de l'État du Piauí.

Autres gisements nordestins. - Piauí (PI): Toca do Garrincho ( ${ }^{\circ}$ 200), Lagoa São Vitor ( $\left.{ }^{\circ} 226\right)$ et Lagoa do Quari ( ${ }^{\circ}$ 607), municipio de São Raimundo Nonato ; ces trois sites sont eux aussi dans la région du Parc National Serra da Capivara. - Ceará (CE) : Sobral. — Paraíba (PB): Fazenda Vista Alegre à Taperoá. - Pernambuco (PE): Lagoa da Pedra à Conceição das Creoulas, municipio de Salgueiro. - Rio Grande do Norte (RN): Lagoa do Santo à Currais Novos, Lagoa da Cruz à Nova Cruz. Tous ces gisements sont du Pléistocène supérieur, certains sont peut-être également de l'Holocène ancien. 


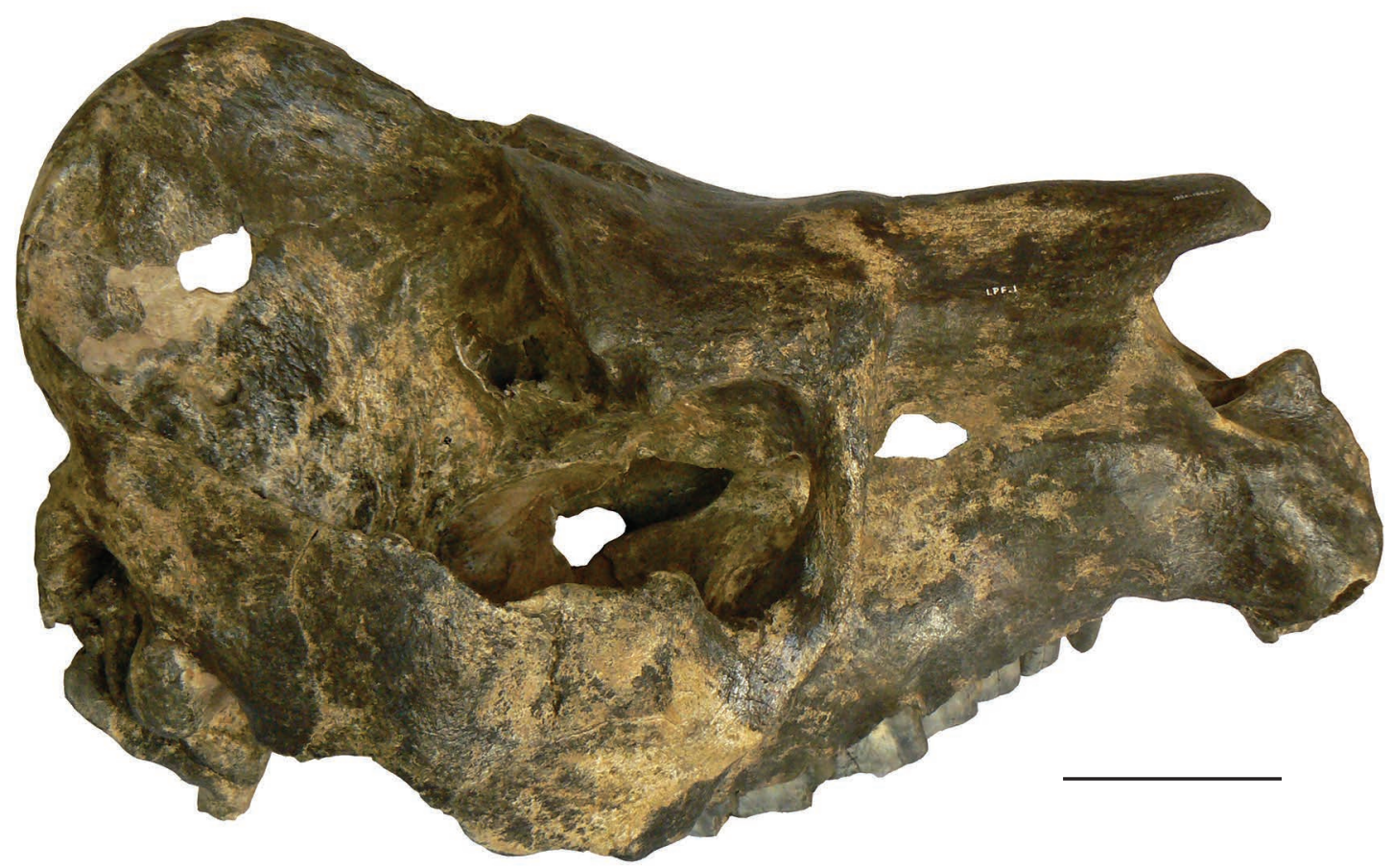

FIG. 1. - Piauhytherium capivarae n. gen., n. sp., Lagoa dos Porcos, crâne $\mathrm{n}^{\circ} 188265$, holotype, vue latérale droite. Échelle : $10 \mathrm{~cm}$.

DiAGNOSE GÉNÉRIQUE ET SPÉCIFIQUE. — Le genre étant monospécifique, la diagnose est commune au genre et à l'espèce. Crâne à frontaux bombés transversalement et longitudinalement en avant, et faiblement déprimés longitudinalement vers l'arrière; crête sagittale courte et épaisse au niveau des pariétaux. Face occipitale subplane fortement inclinée vers l'avant et le haut dans les deux tiers inférieurs de sa partie centrale; au dessus cette face est très creusée. Bords de la symphyse mandibulaire convergents vers l'avant. I/ 3 très forte dont le plus grand diamètre est horizontal. $\mathrm{P} 4$ / avec vallée linguale très étroite ; son périmètre possède trois bandes d'émail, une recouvrant tout l'ectolophe, une autre la moitié linguale de la face antérieure du protolophe, une dernière sur la face antérieure du métalophe, c'est à dire sur tout le bord postérieur de la vallée linguale. M1/ et M2/ avec ectolophe de sub-rectiligne à ondulé ; une bande d'émail recouvre tout l'ectolophe, une autre borde toute la vallée et s'étend vers l'arrière presque jusqu'à l'angle postéro-interne de la dent, une troisième recouvre presque toute la face antérieure du protolophe. $\mathrm{P} / 3$ et $\mathrm{P} / 4$ avec synclinal labial peu profond et synclinal lingual marqué, $\mathrm{P} / 4$ courte et large, à face labiale recouverte d'émail mais dépourvue de bande d'émail linguale médiane. M/1 avec émail sur la partie linguale du métaconide, $\mathrm{M} / 2$ avec trois sillons linguaux dont un petit pli méta-entoconide, $\mathrm{M} / 3$ avec net pli ento-hypoconide. Humérus, métacarpiens, fémur et tibia un peu plus courts que chez Toxodon platensis, métacarpiens à diaphyse relativement plus large et épiphyse distale plus volumineuse.

\section{DESCRIPTION ET COMPARAISONS \\ Crâne}

Nous disposons de quatre crânes plus ou moins complets. Le crâne $n^{\circ} 188265$ de la Lagoa dos Porcos, pratiquement complet, parfaitement conservé et non déformé sert de base à la description qui suit; il est bien adulte et toutes les sutures, sauf une partie de l'interfrontale, sont oblitérées et invisibles. Le crâne ${ }^{\circ} 185391$, lui aussi bien adulte, est dépourvu de son toit crânien et de la partie supérieure de sa face occipitale. Il manque au crâne n ${ }^{\circ} 48947$ de la Lagoa São Vitor toute la partie située en avant de la suture frontal/nasal et frontal/maxillaire, le palais et les dents (quatre sont associées au crâne mais pas en place), le dièdre pariéto-occipital et l'essentiel de la partie droite de la face occipitale, les arcades zygomatiques (sauf la racine postérieure de l'arcade gauche). Le crâne 


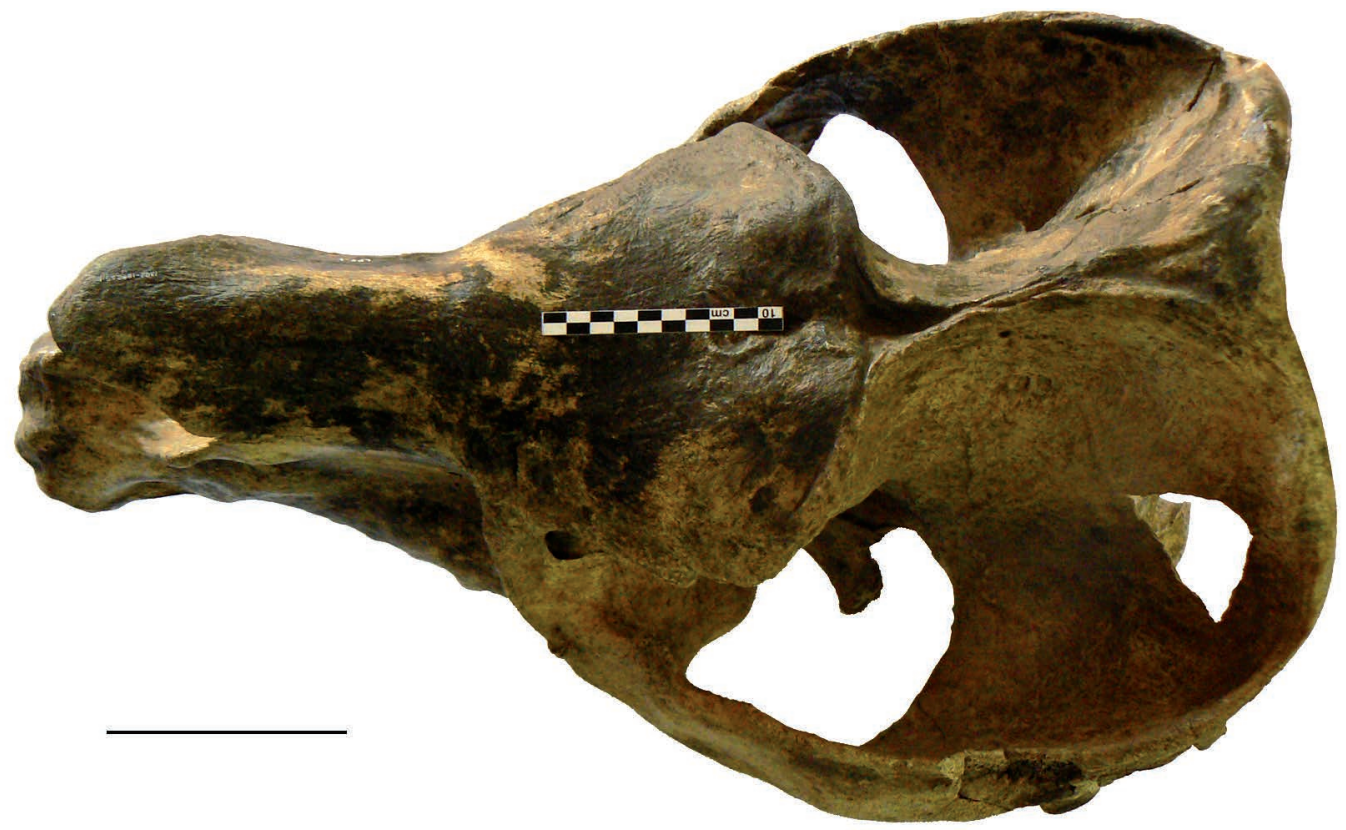

FlG. 2. - Piauhytherium capivarae n. gen., n. sp., Lagoa dos Porcos, crâne n 188265 , holotype, vue supérieure. Échelle : $10 \mathrm{~cm}$.

n 108998-1 de la Lagoa do Quari est écrasé verticalement, sa partie supérieure a été déformée et partiellement détruite, il manque les arcades zygomatiques, le palais, toute la partie antérieure gauche, et la partie antérieure droite en avant de la $\mathrm{P} 4 /$; le maxillaire droit est incomplet et très déformé. En vue latérale (Fig. 1) le profil dorsal est très élevé en arrière au niveau des pariétaux ; vers l'avant, au niveau des frontaux, il devient concave en avant de la suture frontopariétale puis se relève ensuite pour former une petite bosse au niveau de l'avant de l'orbite ; plus en avant il devient faiblement ondulé puis à peu près rectiligne jusqu'au bout des nasaux. Sur le crâne de São Vitor le frontal est plus fortement bombé en avant. Les nasaux sont très courts, le fond de l'échancrure nasale est au niveau de l'extrême avant de P1/. Les prémaxillaires très massifs portent au dessus de leur partie postéro-supérieure une forte tubérosité sagittale. Les pariétaux se rejoignent vers l'arrière pour constituer une crête sagittale courte (sa longueur est de l'ordre de $60 \mathrm{~mm}$ ) et épaisse $(21 \mathrm{~mm}$ ) disposée à angle largement obtus par rapport à l'arrière des frontaux ; la courte crête sagittale et le bombement des frontaux se retrouvent sur le crâne de Quari malgré ses déformations. L'orbite est grande, beaucoup plus haute que longue, ouverte en arrière. Il n'y a pas de processus anté- ni sus-orbitaire, mais un processus post-orbitaire net marque l'arrière de l'orbite ; à une quarantaine de millimètres en arrière de ce dernier, au niveau de la partie la plus externe du frontal, se trouve une petite pointe dirigée vers le bas. Dans la fosse temporale, sur chaque pariétal, se trouve une crête longitudinale fortement convexe vers le haut, qui part au dessus de l'orbite et vient s'ennoyer vers le milieu de la fosse. Le bord antérieur de l'orbite est au niveau de l'intervalle $\mathrm{P} 4 /-\mathrm{M} 1 /$. Un très gros foramen sous-orbitaire, dont le fond se situe au dessus de $\mathrm{P} 4 /-\mathrm{M} 1 /$, se place à mi-hauteur de l'orbite et n'en est séparé que par la bordure antérieure de celle-ci. L'arcade zygomatique est très puissante, sa hauteur maximale est de $132 \mathrm{~mm}$, son dessin est sigmoïde. L'apophyse postglénoïde est épaisse et courte, bien séparée de la post-tympanique qui s'étend un peu plus ventralement. L'apophyse paroccipitale très 


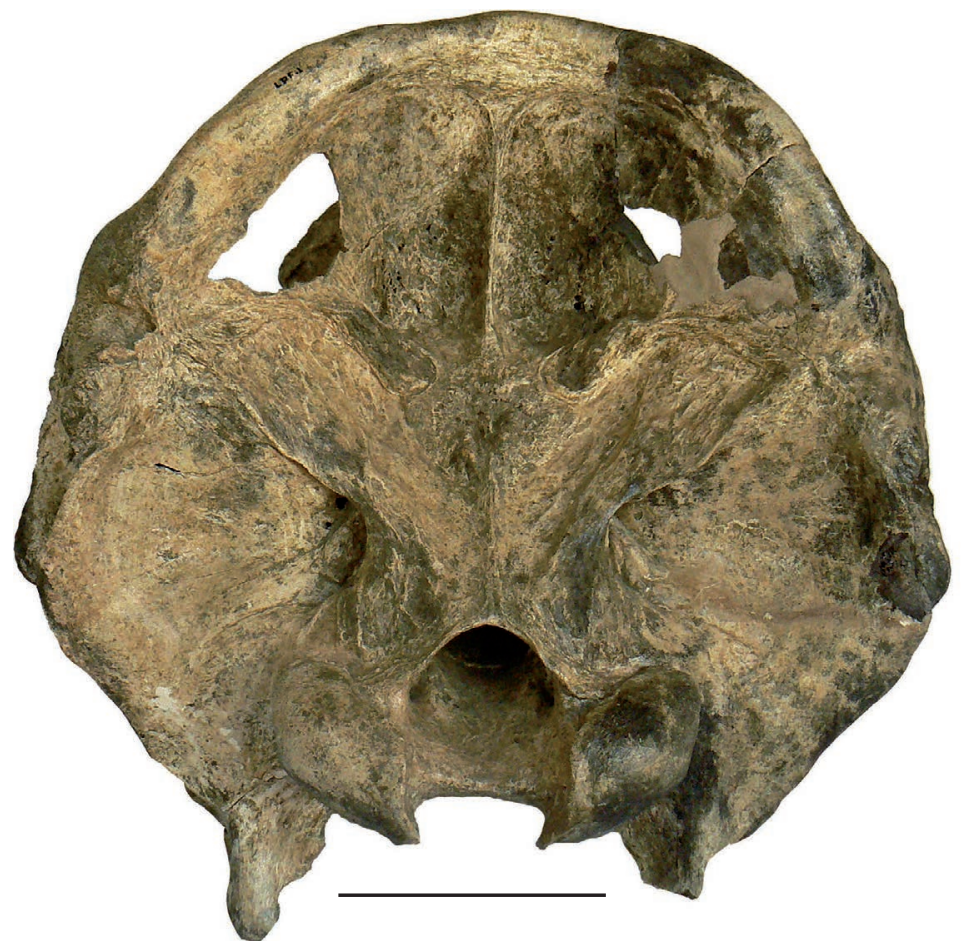

FIG. 3. - Piauhytherium capivarae n. gen., n. sp., Lagoa dos Porcos, crâne $\mathrm{n}^{\circ} 188265$, holotype, vue occipitale. Échelle : $10 \mathrm{~cm}$.

haute descend beaucoup plus ventralement que les deux autres, en dessous du méat auditif fermé elle reste séparée de la post-tympanique par un long sillon vertical profond et étroit.

En vue dorsale (Fig. 2) le contour général est piriforme, très élargi en arrière. Les nasaux s'élargissent vers l'avant jusqu'au niveau du fond de l'échancrure nasale, puis se rétrécissent progressivement jusqu’à leur extrémité antérieure arrondie. Les frontaux dessinent un écusson convexe transversalement et légèrement concave longitudinalement, élargi en arrière, avec une gouttière sagittale dans sa moitié postérieure. L'écusson est séparé des pariétaux par un rebord net incluant un grand trou triangulaire sagittal assez profond. De chaque côté la bordure de l'écusson, légèrement en relief, limite en avant et vers le haut la fosse temporale; les deux bordures se poursuivent sur les pariétaux et confluent en une puissante crête sagittale qui s'élargit vers l'arrière puis se dédouble pour rejoindre de chaque côté la grande crête occipitale. La boîte crânienne très étroite en avant s'élargit vers l'arrière. La grande crête occipitale, déprimée en son milieu, passe en continuité à la bordure latérale de chaque arcade zygomatique où elle s'amincit en se prolongeant jusqu'à l'avant de l'arcade ; sous cet angle elle enferme ainsi tout l'arrière-crâne dans une véritable collerette osseuse, épaisse sur sa bordure mais très mince immédiatement en deçà.

La face occipitale (Fig. 3) a un contour arrondi, avec des apophyses mastoïdes saillantes. Elle est globalement très concave tant verticalement qu'horizontalement. Dans sa partie centrale elle est à peu près plane et, pour ses deux tiers inférieurs, fortement inclinée vers l'avant et le haut. Son tiers supérieur est très déprimé et porte une crête sagittale bien marquée. La face porte un ensemble complexe d'insertions musculaires; dans son tiers inférieur se trouvent deux crêtes abaxiales partant chacune du niveau du bord latéral du condyle occipital ; ces deux crêtes se rapprochent puis divergent en direction de la grande crête occipitale ; au milieu 


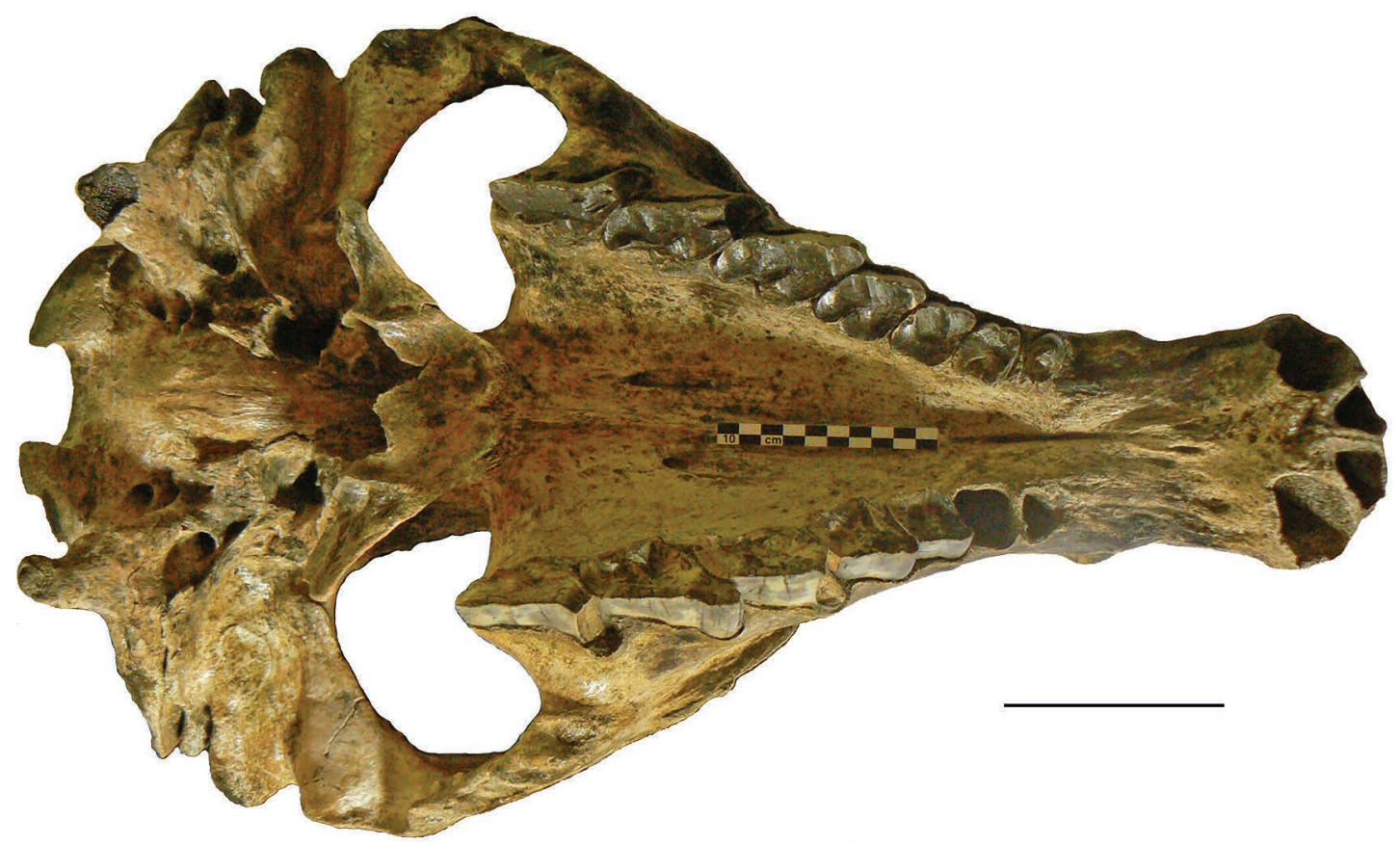

FIG. 4. - Piauhytherium capivarae n. gen., n. sp., Lagoa dos Porcos, crâne $\mathrm{n}^{\circ} 188265$, holotype, vue ventrale. Échelle : $10 \mathrm{~cm}$.

de chacune se trouve un grand foramen perforant toute la paroi osseuse. Le trou occipital est plus ou moins elliptique, les condyles sont aussi puissants que pour un rhinocéros ou un hippopotame. Les apophyses paroccipitales descendent bien en dessous des condyles.

En vue de face l'ouverture nasale est haute et étroite, sa largeur atteint $98 \mathrm{~mm}$. Au niveau de la suture naso-frontale, les frontaux bombés transversalement ont une section en U inversé ; sur le fragment de crâne de São Vitor (cassé au niveau de cette suture) leur épaisseur dans le plan sagittal atteint $33 \mathrm{~mm}$, une telle épaisseur pourrait-elle correspondre à l'insertion d'un phanère ? La tubérosité sur l'arrière des prémaxillaires (large de $22 \mathrm{~mm}$ ) et les alvéoles des quatre incisives sont bien visibles sous cet angle.

En vue ventrale (Fig. 4), les rangées dentaires forment un $V$, le palais, très creusé en $U$, s'étend de chaque côté depuis l'avant des prémaxillaires jusqu'à l'arrière des M3/. Dans sa partie centrale il passe vers l'arrière aux ptérygoïdes qui constitu- ent une plate-forme horizontale en $V$, plus ventrale que le palais. Les prémaxillaires (Fig. 5) sont à peine élargis au niveau des alvéoles des I2/ chez P. capivarae n. gen., n. sp., mais très élargis pour T. platensis chez qui la forme des alvéoles est très différente. Les basi-occipitaux sont larges, renflés transversalement et longitudinalement. Les dimensions sont données en Annexes (Tableau 1). Elles sont du même ordre de grandeur que celles de T. platensis publiées par Miño Boilini et al. (2006). En vue latérale le profil du toit crânien est très différent de celui de Toxodon platensis chez qui frontaux et pariétaux sont alignés sagittalement dans un même plan plongeant doucement vers l'avant et le bas, et font un angle presque droit avec la face occipitale verticale. Toxodon platensis est aussi très différent par le grand élargissement de ses prémaxillaires et la disposition alignée des alvéoles de ses incisives. Il diffère également de $P$. capivarae $n$. gen., n. sp. en vue ventrale par ses basioccipitaux moins rétrécis vers l'avant et par la surface des ptérygoïdes située au même niveau que la surface des palatins. 

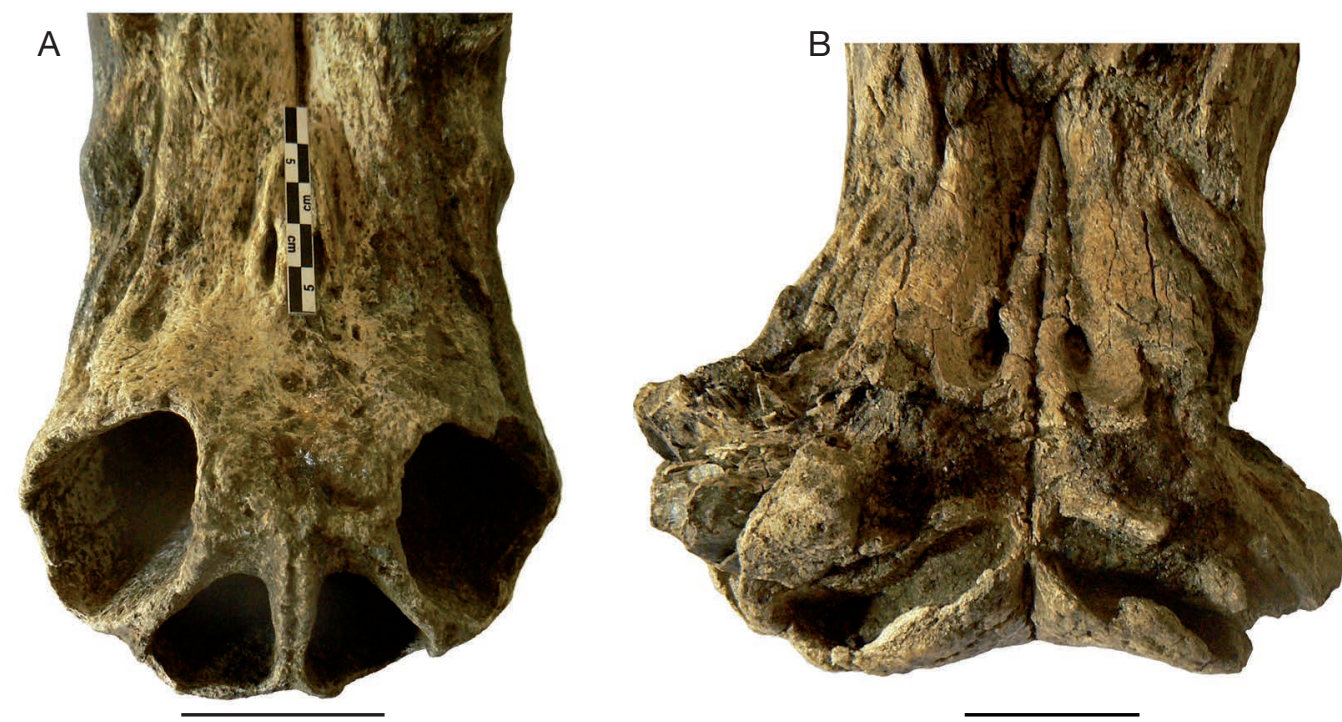

FIG. 5. - Prémaxillaires, vue ventrale, crânes n 188265 et $n^{\circ} 186001$, Lagoa dos Porcos : A, Piauhytherium capivarae n. gen., n. sp., $\mathrm{n}^{\circ} 188265$, holotype; B, Toxodon platensis n 186001 . Échelles : $5 \mathrm{~cm}$.

Mandibule. Nous disposons de deux mandibules plus ou moins complètes. La mandibule $\mathrm{n}^{\circ} 187141$ de la Lagoa dos Porcos (Fig. 6) a été découverte à proximité du crâne $\mathrm{n}^{\circ} 188265$ et lui correspond très probablement. Elle est à peu près complète pour sa partie gauche à laquelle il ne manque que l'apophyse coronoïde ; à droite la branche montante n'a pas été conservée et la $\mathrm{M} / 3$ a disparu. Le fragment de mandibule de São Vitor comporte la branche horizontale droite depuis la partie arrière de la symphyse jusqu'à l'avant de la branche montante, et une partie de la rangée dentaire $(\mathrm{P} / 4$ à $\mathrm{M} / 3)$. La branche horizontale massive s'amincit progressivement vers l'avant. Son bord ventral est faiblement convexe longitudinalement avec une inflexion maximale au niveau de l'arrière de $\mathrm{M} / 2$ et de l'avant de M/3. Les bords latéral et médial sont presque plats. Le foramen mentonnier proche du bord ventral se situe entre $\mathrm{P} / 4$ et $\mathrm{M} / 1$. La branche montante a son bord antérieur faiblement oblique vers l'avant et le haut. Son bord postérieur est nettement et régulièrement convexe. En vue de dessus le condyle faiblement convexe vers l'avant présente un contour conique, avec une extrémité médiale bien plus épaisse que la latérale. La symphyse très longue et très haute possède un bord postérieur en $S$ dont le point le plus caudal se situe entre $M / 1$ et $M / 2$; la partie supérieure de ce bord n'est qu'au niveau du quart antérieur de M/1. Il n'y a pas de carène ventrale. Les bords droit et gauche sont dilatés au niveau des I/2 (le diamètre transversal atteint $98 \mathrm{~mm}$ à ce niveau) puis convergent vers l'avant, ce qui montre que le muffle de Piauhytherium n. gen. était beaucoup plus étroit que celui de Toxodon (Fig. 7), avec un positionnement des incisives très différent. Les dimensions sont données en Annexes (Tableau 2). La branche horizontale est globalement plus basse et plus étroite que pour Toxodon platensis chez qui en outre la partie rostrale de la symphyse est beaucoup plus large, et dont le bord postérieur de la symphyse est en général au niveau du milieu de $M / 1$, par exemple sur la très belle mandibule d'Argentine $n^{\circ} 28$ conservée au Zoologisk Museum de Copenhague.

Chez Trigodonops lopesi la branche horizontale est plus large et surtout beaucoup plus haute (153 mm devant M/1 selon Roxo (1921) contre 113 à 118 mm). 


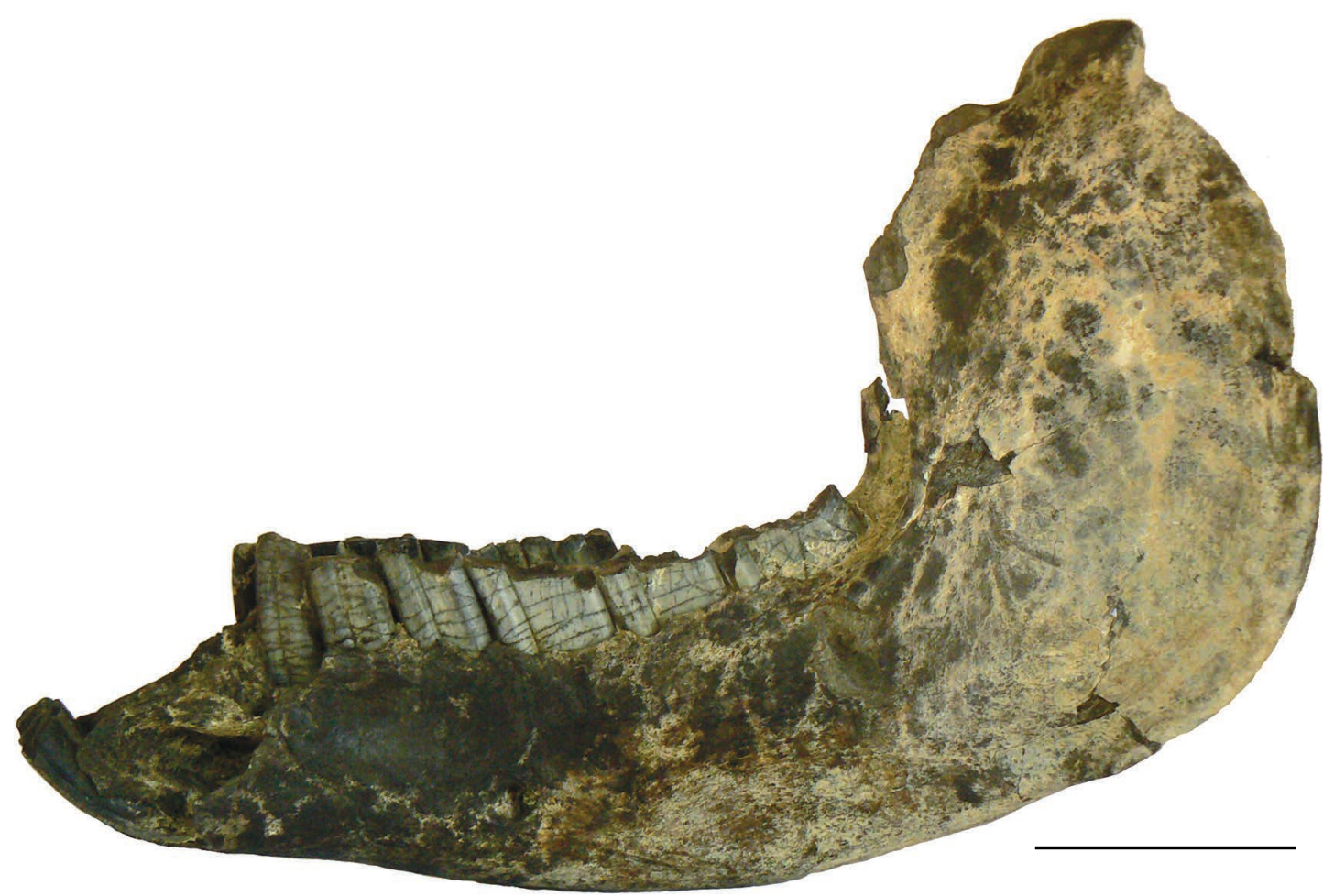

FIG. 6. - Piauhytherium capivarae n. gen., n. sp., Lagoa dos Porcos, mandibule $\mathrm{n}^{\circ} 187141$, vue latérale gauche. Échelle : $10 \mathrm{~cm}$.

Chez Mixotoxodon le bord postérieur de la symphyse atteint le bord antérieur ou la moitié de M/1 (van Frank 1957: 7), Le bord antérieur de la branche montante est à peu près perpendiculaire au plan occlusal, la branche montante est moins arrondie, le condyle est nettement plus bas et la disposition des incisives est différente (van Frank 1957: figs 4, 5).

\section{Denture}

La formule dentaire adulte de $P$. capivarae n. gen., n. sp. est 2/3I, 0/0 C, 4/4 P, 3/3 M, les I2/ et I/3 étant transformées en défenses. C'est la même formule que chez T. platensis. Pour les dents inférieures la formule est identique chez $M$. larensis. Les jugales de $P$. capivarae n. gen., n. sp. et T. platensis sont dépourvues de racines, celles de Mixotoxodon ont des racines ouvertes (van Frank 1957: 7); Cisneros (2005: 246) fait lui aussi état de la présence de racines dans ce dernier genre.
Incisives supérieures. Les incisives du crâne $\mathrm{n}^{\circ} 188265$ de la Lagoa dos Porcos sont toutes tombées, mais leurs alvéoles sont parfaitement conservées ; celles du crâne ${ }^{\circ} 185391$ sont conservées et sont en assez bon état à droite ; elles sont recourbées vers le bas.

I1/ est à section en triangle rectangle avec l'hypoténuse disposée latéralement. Le DT de l'alvéole est de $25 \mathrm{~mm}$ pour un DAP de $33 \mathrm{~mm}$. Pour les I1/ du crâne $n^{\circ} 185391$ ces dimensions sont respectivement 24 et $26 \mathrm{~mm}$; le bord antérieur et le bord médial sont recouverts d'émail.

I2/ est fortement recourbée vers le bas. Sa section est en triangle aplati étiré transversalement, à pointe médiale ; le DT atteint $49 \mathrm{~mm}$, le DAP côté latéral $25,5 \mathrm{~mm}$; l'émail est présent sur la face antérieure et sur la face dorsale, mais absent entre les deux sur l'angle antéro-latéral du triangle. La disposition des incisives supérieures est très différente de celle de T. platensis (Fig. 5). 


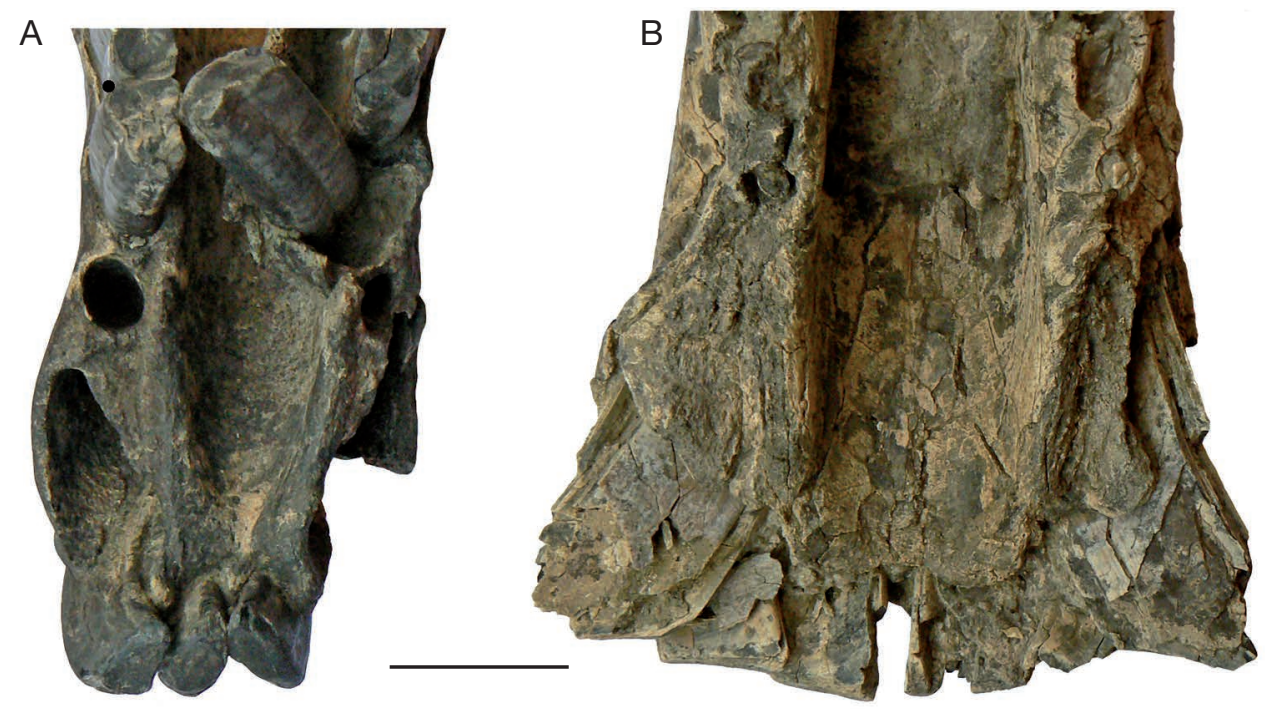

FIG. 7. - Partie symphysaire, vue dorsale, des mandibules, Lagoa dos Porcos : A, Piauhytherium capivarae n. gen., n. sp., holotype, $\mathrm{n}^{\circ} 187141$; B, Toxodon platensis Owen, $1837 \mathrm{n}^{\circ} 186001$. Échelle : $5 \mathrm{~cm}$.

Chez ce dernier, I1/ est étirée transversalement sur $56 \mathrm{~mm}$, sa section est presque réniforme ; I2/ large de plus de $60 \mathrm{~mm}$ est en triangle aplati, à pointe médiale, elle est disposée immédiatement en arrière de I1/, parallèlement à celle-ci et en partie plus latéralement.

Jugales supérieures. Les deux crânes de la Lagoa dos Porcos ont conservé leurs rangées dentaires, celles du crâne n ${ }^{\circ} 188265$ sont représentées Figure 8. Les dimensions comparées de ces dents sont données en Annexes (Tableau 3). Sur les rangées dentaires du crâne $\mathrm{n}^{\circ} 185391$ les bandes d'émail sont bien visibles. Sur le crâne écrasé de Quari il ne subsiste plus que les trois molaires droites, précédées de la quatrième prémolaire basculée vers l'intérieur, toutes sont mal conservées. Quatre autres jugales supérieures, isolées, ont été recueillies dans le même site. Garrincho en a livré sept. Le crâne de São Vitor est associé à quatre dents jugales. Des jugales supérieures isolées ont été recueillies dans presque tous les sites.

- P1/: elle a une section en ellipse à grand axe transversal. Une couche d'émail recouvre la face avant et passe sur la face labiale qu'elle recouvre entièrement.
- P2/: elle est bilobée avec un net synclinal lingual mais ne possède pas de vallée interne. Lémail recouvre la moitié linguale de la face antérieure et toute la face labiale. Deux spécimens de Garrincho présentent un ectolophe échancré en son centre par un sillon plus fort qu'à la Lagoa dos Porcos. - P3/ : elle a le même contour que P2/ ; la face linguale est faiblement déprimée en son milieu mais ne comporte pas de vallée. L'ectolophe est lui aussi faiblement déprimé en son milieu. Il existe deux bandes d'émail, l'une étendue sur tout l'ectolophe, l'autre sur la moitié linguale du bord antérieur de la dent. Une P3/ de Garrincho se distingue par son ectolophe plus fortement ondulé.

- P4/: c'est la seule prémolaire présentant une vallée linguale, par ailleurs extrêmement étroite et s'étendant presque jusqu'au milieu de la dent. L'ectolophe est faiblement ondulé avec une très légère dépression en son milieu. Le périmètre comporte trois bandes d'émail, une très large recouvrant tout l'ectolophe qui déborde légèrement vers l'avant sur le protolophe (la face antérieure du paracône) mais n'atteint pas vers l'arrière la face postérieure du métacône, une autre s'étendant sur la moitié linguale ou les deux tiers linguaux de la face antérieure du protolophe, une dernière sur tout le bord 
postérieur de la vallée linguale. Cette disposition est légèrement différente sur la P4/ isolée de Quari $n^{\circ} 108787$ et la $\mathrm{P} 4 / \mathrm{n}^{\circ} 116193$ de Sáo Vitor où l'émail de la troisième bande, très étroite, ne s'étend que très peu sur le bord postérieur de la vallée ; la variabilité individuelle porte aussi sur la largeur de la bande située sur le protolophe.

- M1/: la section de la dent est en triangle à pan coupé vers l'arrière. L'ectolophe est très légèrement ondulé, avec une petite dépression verticale en arrière du paracône et une autre en son milieu. Le protolophe possède un bord antérieur ondulé avec deux dépressions verticales, l'une bordant l'avant du paracône, l'autre l'avant du protocône. La vallée linguale est longue et large, elle est limitée vers l'arrière par un métalophe massif. Une bande d'émail recouvre tout l'ectolophe sauf sa partie la plus postérieure, une autre borde toute la vallée et s'étend vers l'arrière presque jusqu'à l'angle postéro-interne de la dent, une troisième recouvre presque toute la face antérieure du protolophe sauf ses extrémités. - M2/ : elle est assez semblable à M1/ mais sa partie postérieure plus étirée en arrière n'est pas à pan coupé. Ses dimensions sont un peu plus fortes. L'ectolophe est sub-rectiligne. Le métalophe se distingue bien de l'extension postérieure de l'ectolophe grâce à une dépression du bord lingual de la dent. La bande d'émail linguale commence avec le bord postérieur de la vallée interne et s'étend loin en arrière, presque jusqu'au bord postéro-interne. Le spécimen de Garrincho n³5898 se distingue par son ectolophe plus ondulé et la dépression marquant la limite postérieure du métalophe, qui est ici à angle vif.

- M3/: elle ressemble à une $\mathrm{M} 2$ / encore plus étirée vers l'arrière ; la bande d'émail linguale l'est en revanche beaucoup moins et se limite vers l'arrière au bord lingual du métalophe ; l'ectolophe est subrectiligne ou faiblement ondulé. Un sillon vertical bien plus profond et étroit qu’à la M2/ limite le protocône (qui n'est pas recouvert d'émail) vers l'avant et vers l'extérieur.

Chez Mixotoxodon la P3/ est morphologiquement très semblable à celle de Piauhytherium n. gen., avec toutefois une moindre extension de l'émail sur l'arrière de l'ectolophe. La P4/ présente (van Frank 1957: fig. 8D, G) un ectolophe plus régulier, légèrement convexe; la vallée linguale est étroite et entièrement bordée d'émail. La M1/, plus étirée vers l'arrière, se distingue aussi par son ectolophe fortement ondulé et son protolophe déprimé en avant. Les trois molaires figurées par Laurito (1993: fig. 4) montrent un ectolophe plus ou moins faiblement ondulé ; l'émail est réparti selon trois bandes, une linguale depuis le bord antérieur du protocône jusqu'au métacône (sans en atteindre l'extrémité postérieure), une antérieure entre protocône et paracône (sans toucher à leurs extrémités), une labiale entre le paracône et la face linguale du métacône. Si le spécimen figuré par van Frank (1957: fig. 8E) correspond bien à une molaire de Mixotoxodon cette dent possède un ectolophe rectiligne et se termine vers l'arrière par le même pan coupé que celle de Piauhytherium n. gen. ; sa vallée linguale est étroite. Le bord antérieur du paracône n'est pas souligné par une dépression.

Selon Paula Couto (1982: 20, fig. 24) la P2/ de Trigodonops lopesi de Pedra Preta (Acre), seule dent jugale supérieure connue jusqu' ici pour ce taxon, a à peu près la même taille que celle de Mixotoxodon, mais en diffère surtout parce qu'elle possède un profond repli d'émail au milieu de sa face labiale, alors que chez Mixotoxodon il n'y a qu'une faible sinuosité en cet endroit.

Chez Toxodon platensis la P3/ dispose d'une vallée linguale. La P4/ se distingue par sa vallée linguale plus ouverte entièrement bordée d'émail. La M3/ a un profil d'ectolophe faiblement concave et la limite postérieure de la bande linguale d'émail est plus proche de l'angle postéro-interne de la dent. Selon van Frank (1957: 5) il n'y aurait pas de métalophe aux M3/ de Toxodon alors qu'il y en aurait un aux deux premières molaires, et ce serait un caractère typique des Toxodontinae.

Incisives inférieures. La mandibule $\mathrm{n}^{\circ} 187141 \mathrm{de}$ la Lagoa dos Porcos a conservé ses I/ 1 et / 2 mais les I/3 sont tombées, laissant des alvéoles dont le droit, en excellent état, montre que la dimension maximale de leur section est verticale. Les six incisives, courbées vers le haut, sont disposées en secteur de cercle (Fig. 7).

- I/1 : elle a une section en triangle rectangle dont l'hypoténuse est latérale. Le DT est $18 \mathrm{~mm}$ pour un 


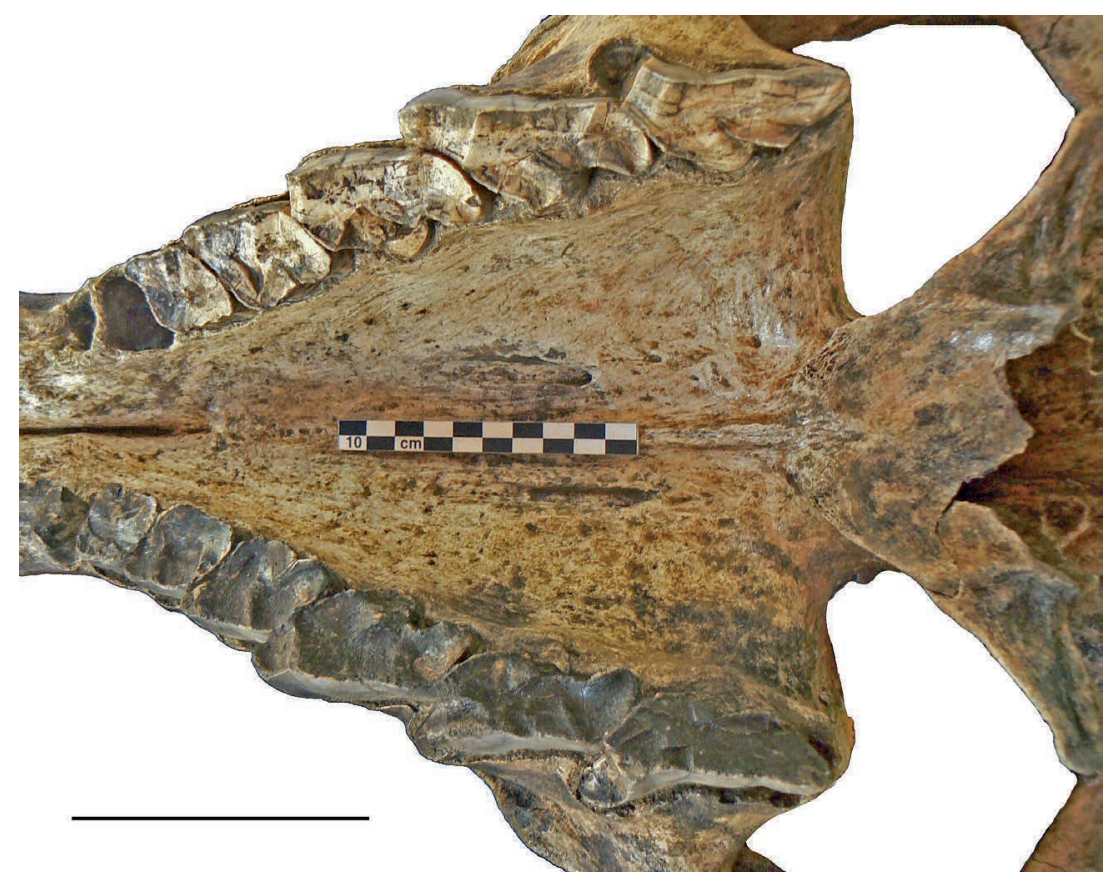

FIG. 8. - Piauhytherium capivarae n. gen., n. sp., Lagoa dos Porcos, rangée dentaire supérieure du crâne $\mathrm{n}^{\circ} 188265$, vue occlusale. Échelle : $10 \mathrm{~cm}$.

DAP de 30,5 mm. Une couche d'émail débordant très peu sur les côtés recouvre la face antérieure, et une petite bande d'émail de $5,5 \mathrm{~mm}$ de large se situe près de l'arrière de la face latérale.

$-\mathrm{I} / 2$ : elle a une section subtriangulaire, son DT est $16 \mathrm{~mm}$ et son DAP $28,5 \mathrm{~mm}$. La face antérieure est recouverte d'émail et il en existe une petite bande postéro-latérale comme pour la M/1. Le fragment d'incisive $n^{\circ} 37001$ de Garrincho appartient probablement à une $\mathrm{I} / 2$ de Piauhytherium $\mathrm{n}$. gen.

- I/3: l'alvéole droit de la I/3 de la mandibule $\mathrm{n}^{\circ} 187141$ est triangulaire et très gros $(35 \times 49 \mathrm{~mm})$. Nous déterminons comme une I/3 droite de Piauhytherium n. gen. la dent ${ }^{\circ} 35844$ de Garrincho. C'est un gros fragment de plus de $160 \mathrm{~mm}$ de long, à peu près droit longitudinalement mais se recourbant vers l'extérieur et se terminant par un biseau d'usure pointu latéralement, à surface légèrement convexe ; la section est triangulaire, le plus grand côté porte de l'émail sur presque toute son extension, sauf dans l'angle supéro-médial ; il y a de l'émail sur l'angle supéro-latéral, qui constitue un dièdre aigu et tranchant, et cet émail passe directement sur le côté latéral, dont il recouvre les deux tiers externes. Il n'y a pas d'émail sur le côté médial ni sur les angles inféro-médial et supéro-médial. La dent n ${ }^{\circ} 35975$ de Garrincho est une I/3 gauche appartenant probablement au même individu que la précédente.

Chez T. platensis la disposition des incisives inférieures n'est pas la même, elles sont sub-horizontales et insérées presque dans le même plan, et la partie antérieure de la symphyse a des bords divergents (Fig. 7) ; elles présentent des sections et une répartition de l'émail bien différentes. La I/1 est en triangle rectangle aplati $(\mathrm{DT} \times \mathrm{DDV}=36 \times 18,5 \mathrm{~mm}$ pour le spécimen $\mathrm{n}^{\circ} 186001$ de la Lagoa dos Porcos) avec l'angle droit antéro-médial et l'hypoténuse en position postérolatérale, la face antérieure est recouverte d'émail, et une étroite bande d'émail se trouve sur l'avant de la face médiale. La I/2 est crescentiforme (DT $\times$ DVD $=37,5 \times 12,5 \mathrm{~mm}$ pour le même individu), la face ventrale convexe est entièrement recouverte d'émail. La I/3 est une puissante défense en triangle arrondi (DT $\times$ DDV environ $49 \times 31 \mathrm{~mm}$ ), avec de l'émail 


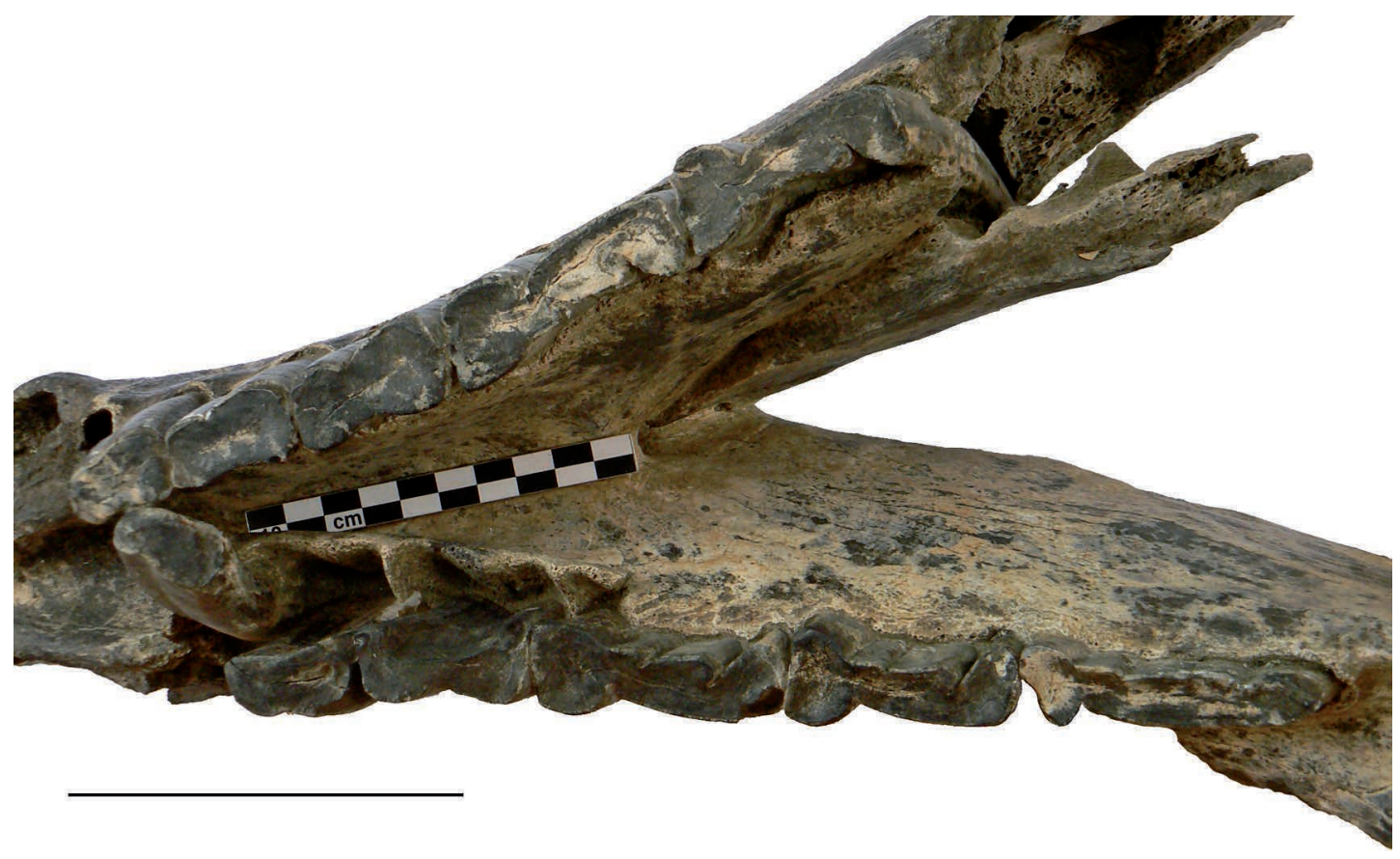

FIG. 9. - Piauhytherium capivarae n. gen., n. sp., Lagoa dos Porcos, rangées dentaires inférieures de la mandibule $\mathrm{n}^{\circ} 187141$, vue occlusale. Échelle : $10 \mathrm{~cm}$.

sur la face antérieure et la plus grande partie de la face médiale, et une petite bande d'émail dans l'angle postéro-médial. Les incisives inférieures du T. platensis de la Lagoa dos Porcos sont identiques à celles du spécimen de La Plata n 28 du Zoologisk Museum de Copenhague, qui est remarquablement conservé.

Chez Mixotoxodon larensis, la partie symphysaire n'est pas divergente vers l'avant et les incisives sont insérées en secteur de cercle, comme chez P. capivarae n. gen., n. sp., mais les troisièmes incisives sont différentes. Les $\mathrm{I} / 1$ et $\mathrm{I} / 2$ disposent de deux bandes d'émail bien séparées, l'une occupant toute la face ventrale et l'autre, très étroite, une partie de la face dorso-latérale (van Frank 1957: fig. 7). La I/3 a une section plus réniforme, elle ne présente pas de dièdre tranchant, son émail réparti en deux bandes séparées, une labiale large et une postéro-linguale étroite (van Frank 1957: 6, figs 5, 7). Elle est beaucoup plus petite (environ $27 \times 13 \mathrm{~mm}$ contre $35 \times 49 \mathrm{~mm}$ ) et sa plus grande dimension est horizontale (van Frank 1957: 6, tableau 2), alors qu'elle est verticale chez P. capivarae n. gen., n. sp.
Jugales inférieures. La mandibule $\mathrm{n}^{\circ} 187141$ de la Lagoa dos Porcos possède toutes ses dents jugales sauf les P/1 (dont les alvéoles sont conservés) et la M/3 droite (Fig. 9). Nous avons aussi du même site un fragment d'hémimandibule gauche ${ }^{\circ} 186943$ portant P/3-M/3. L'hémimandibule droite de São Vitor porte la dernière prémolaire et les trois molaires (Fig. 10). Outre de nombreux fragments non étudiables, deux jugales inférieures isolées complètes ont été découvertes à Garrincho et une seule à Quari. Leurs dimensions comparées sont données en Annexes (Tableau 4).

- P/1 : l'alvéole de cette dent est sub-circulaire, d'environ $16 \mathrm{~mm}$ de diamètre.

- $\mathrm{P} / 2$ : elle est bilobée grâce à deux dépressions verticales, une côté labial et une côté lingual; le lobe antérieur est plus étroit. La face labiale est recouverte d'émail.

- P/3: la face labiale est entièrement recouverte d'émail, elle porte un sillon vertical bien marqué mais très étroit et peu profond qui divise la dent en deux lobes ; le lobe antérieur est nettement plus 


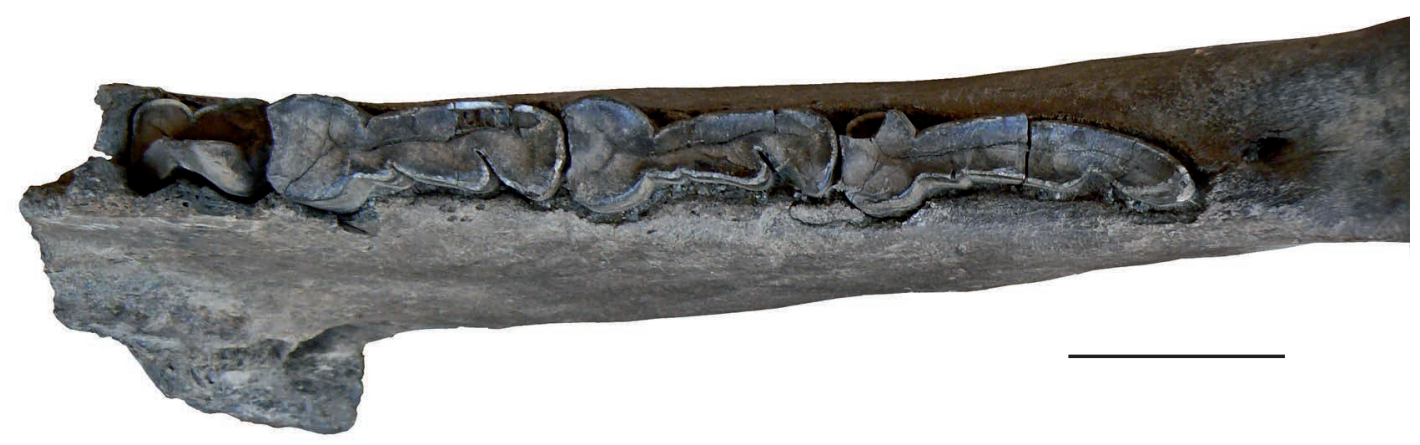

FIG. 10. - Piauhytherium capivarae n. gen., n. sp., Lagoa São Vitor, rangée dentaire inférieure P/4-M/3 de l'hémimandibule droite $\mathrm{n}^{\circ}$ 48061, vue occlusale. Échelle : $5 \mathrm{~cm}$.

court et plus étroit que le postérieur. La face linguale est déprimée et ne porte pas d'émail.

- P/4 : elle est bilobée comme la précédente et le lobe postérieur est plus large que l'antérieur. La face labiale est entièrement recouverte d'émail ; le synclinal labial séparant les deux lobes est peu profond ; la face linguale, totalement dépourvue d'émail, porte une faible dépression verticale médiane.

- M/1 : la face labiale entièrement revêtue d'émail comporte deux lobes séparés par un sillon vertical (le pli labial) assez profond et étroit, qui borde le protoconide en arrière. La face linguale compte quatre lobes successifs, avec un synclinal peu profond (pli antérieur) séparant paraconide et métaconide, un petit sillon étroit (pli métaentoconide) entre métaconide et entoconide, et un sillon tout aussi étroit mais beaucoup plus profond entre entoconide et hypoconide (pli entohypoconide). En face linguale seuls les trois lobes postérieurs et les plis qui les séparent portent de l'émail, qui recouvre donc tout le deuxième et le troisième lobes (métaconide et entoconide) et l'avant du dernier (hypoconide). L'émail manque donc sur la moitié linguale de la face antérieure, sur le bord lingual du paraconide et sur l'angle postéro-lingual de la dent.

$-M / 2$ : elle est morphologiquement identique à la M/1 dont elle ne se distingue que par un sillon métaentoconide à peine indiqué et par des proportions un peu différentes: la largeur postérieure semble nettement plus faible que la largeur antérieure, alors que leurs valeurs sont proches sur la M/1.
- M/3 : la partie postérieure est très allongée et la dent se termine en pointe qui s'incurve légèrement vers l'intérieur. À ce détail près la morphologie et la répartition de l'émail sont très proches de la $\mathrm{M} / 2$, mais le sillon méta-entoconide est plus large et encore moins profond, alors que le sillon ento-hypoconide est profond et surtout remarquablement large.

Chez Trigodonops les $\mathrm{P} / 3$ et $\mathrm{P} / 4$ se distinguent par leur sillon labial beaucoup plus profond atteignant largement le milieu de la dent, leur lobe antérieur est petit et arrondi, leur face linguale est légèrement excavée. La $\mathrm{P} / 4$ porte au milieu de sa face linguale une bande verticale d'émail large de $1 \mathrm{~cm}$; la P/4 du T. lopesi de Pedra Preta (Acre) porte, comme l'holotype, cette bande d'émail caractéristique (Paula Couto 1982: fig. 21). La M/1 de l'holotype de Trigodonops ne possède pas de sillon entre métaconide et entoconide. Pour les M/1 de T. lopesi de Pedra Preta, Paula Couto (1982: 18-20, figs 20,23) note l'extension de la bande d'émail de la face linguale sur le métaconide jusqu'à la ligne transversale passant par le pli aboral ; par ailleurs la M/1 de sa figure 20 ne porte d'émail que sur moins de la moitié labiale de sa face postérieure. Paula Couto remarque aussi la présence d'un repli métaconide qui est absent sur l'holotype de T. lopesi. Sur l'holotype de Trigodonops la M/2, tout comme la $\mathrm{M} / 1$, est dépourvue de sillon méta-entoconide (il n'y a qu'une petite ondulation à ce niveau). Comme pour M/1 il n'y a pas d'émail autour du paraconide ni sur la partie linguale de l'hypoconide. Entre 
l'ondulation qui remplace le pli méta-entoconide et le lobule antéro-interne proéminent se trouve un petit relief, plus marqué à la $\mathrm{M} / 2$, ce qui justifie l'affirmation de Roxo (1921) comme quoi la M/2 a une face interne quadrilobée. La dent de Cachoeira da Pedreira (Acre) décrite comme M/2 par Paula Couto (1982: 19) se caractérise par son talonide élargi en arrière, la présence d'un faible repli méta-entoconide, son repli ento-hypoconide fort et recourbé en avant à son extrémité antérieure. Le dessin par Roxo du contour de M/2 contient selon Kraglievich (1931) une fausse prolongation sur le lobule antérieur de la face linguale de la double ligne marquant la présence d'émail. Kraglievich pensait que toute la face linguale de ce lobule devait manquer d'émail, comme c'est le cas pour ce même lobule à la $\mathrm{M} / 1$.

La M/3 de Cachoeira da Pedreira attribuée à Trigodonops par Paula Couto (1982: 19 ; fig. 22) se caractérise par son talonide très allongé dont la partie postérieure est plus étroite que sa partie moyennne, son repli méta-entoconide faible, son repli ento-hypoconide absent et remplacé par une concavité simple mais forte sur la face linguale de la dent. Il y a du cément. Dans la diagnose originelle publiée en 1921, les dimensions données par Roxo (P/4: $35 \times 15 \times 18 \mathrm{~mm} ; \mathrm{M} / 1: 44 \times$ $17 \times 16 \times 18 \mathrm{~mm} ; \mathrm{M} / 2: 46 \times 20 \times 15 \times 15 \mathrm{~mm}$ ) sont correctes pour les dents prises isolément, en dépit d'une double confusion entre longueur et hauteur et entre width et length, mais celles des segments dentaires sont fausses, notamment les longueurs des ensembles des deux dernières prémolaires et des deux premières molaires (Kraglievich 1930, 1931).

La M/2 d'Abothrodon est la seule jugale inférieure connue pour ce genre. Cette dent est très simplifiée avec une face linguale totalement dépourvue de plis ou fossettes, de telle façon que le pilier de l'entoconide n'est pas isolé.

Les jugales inférieures de Mixotoxodon larensis se distinguent de celles de Piauhytherium n. gen. par la présence chez le premier d'un synclinal profond au milieu du bord labial des P/4, l'absence d'émail sur la partie linguale du métaconide des $\mathrm{M} / 1$, l'absence d'un petit pli méta-entoconide aux $\mathrm{M} / 2$ et l'absence d'un pli ento-hypoconide aux $\mathrm{M} / 3$.
Chez Toxodon platensis, les $\mathrm{P} / 4$ ne portent aucun sillon, leur face labiale est seulement faiblement déprimée, la face linguale porte une faible dépression verticale médiane. Les $\mathrm{M} / 1$ et $\mathrm{M} / 2$ ont un pli labial fort mais peu profond, bordant le protoconide sur l'arrière, et deux profonds plis labiaux d'extension à peu près égale, un méta-entoconide et un entohypoconide. Ces dents sont entourées d'émail sauf sur l'avant du paraconide, sur leur face postérieure et sur la partie la plus linguale de l'hypoconide. La M/3 est construite sur le même plan mais son extrémité postérieure est plus allongée et plus étroite ; le sillon ento-hypoconide n'est pas plus large ni plus profond que le sillon méta-entoconide.

\section{Humérus}

Nous avons attribué à $P$. capivarae n. gen., n. sp. six humérus plus ou moins complets. Deux des trois spécimens de Quari sont sub-complets mais leur épiphyse proximale est endommagée; il en est de même pour un spécimen de la Lagoa dos Porcos. Nous rapportons aussi à $P$. capivarae n. gen., n. sp. une épiphyse distale de Garrincho et un humérus incomplet de la région de Sobral (Ceará).

L'allure générale de l'humérus de P. capivarae n. gen., n. sp. est très caractéristique de la famille. Il est court et large, avec un rétrécissement maximum au niveau du milieu de la diaphyse. La tête n'occupe que le tiers médial de l'épiphyse proximale. La coulisse bicipitale est très petite, mais le grand trochanter est énorme. Sur l'arrière de l'épiphyse distale la fosse olécrânienne est très large et très profonde. Sur le spécimen n ${ }^{\circ} 108981$ de Quari le fond de la fosse est naturellement perforé par un trou de $27 \mathrm{~mm}$ de diamètre. Un rebord sépare l'arrière de l'articulation distale de la fosse olécrânienne. L'articulation de l'épiphyse distale est très étroite par rapport à la largeur de celle-ci. Le bord latéral de cette épiphyse, non articulaire, s'étend à environ $45^{\circ}$ de la tangente à la surface articulaire.

Nous avons étudiés deux humérus de T. platensis provenant du Pernambuco, un de Laje Grande à Pesqueira, conservé au Museu nacional de Rio, et un de Quixabinha à Petrolândia. Ce dernier figuré par Rolim en 1974 (qui l'avait rapporté avec doute à Trigodonops) est conservé à l'UFPE. Ils se situent tous deux dans les limites de variation d'un échantillon de T. platensis argentins (Annexes, Tableau 5). 

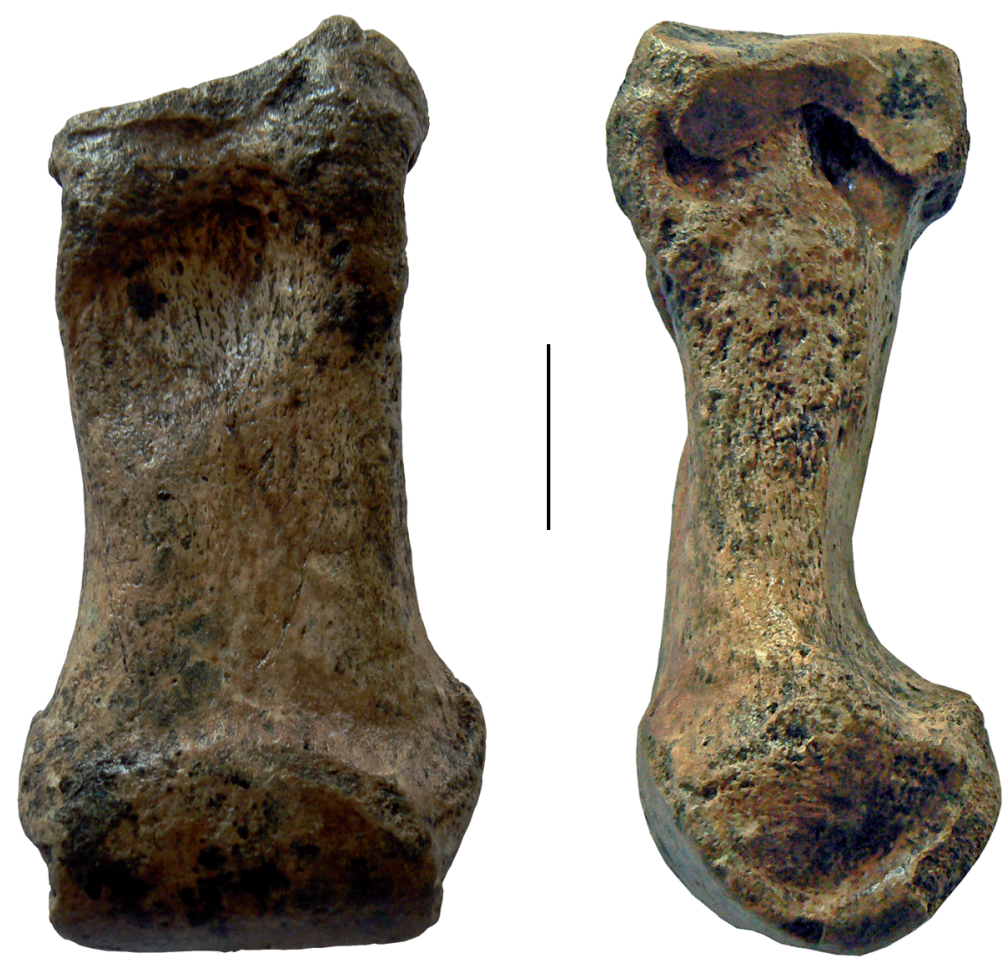

FlG. 11. - Piauhytherium capivarae n. gen., n. sp., Lagoa do Quari, Mc Ill gauche n 109005 : A, vue de face; B, vue latérale. Échelle : 3 cm.

Nous n'avons pas retrouvé au Museu nacional de Rio l'humérus gauche complet de Pesqueira (PE) décrit et figuré par Vidal (1959). Cet auteur note que la diaphyse très courte est tordue sur son axe, que le grand trochanter est très développé, que la crête deltoïde est relativement faible, que l'épiphyse distale comprimée d'avant en arrière est très élargie et que la fosse olécrânienne est relativement petite. Dès 1855 Gervais avait insisté sur les ressemblances morphologiques de l'humérus avec ceux des rhinocéros et des hippopotames, sur la coulisse bicipitale saillante et la puissance des épiphyses.

Les dimensions sont voisines des valeurs minimales relevées sur notre échantillon de comparaison de T. platensis. Les proportions des deux échantillons sont assez semblables, mais l'humérus de $P$. capivarae $\mathrm{n}$. gen., n. sp. est en moyenne plus court et possède une diaphyse plus trapue et une épiphyse distale relativement moins volumineuse.

\section{Cubitus}

Deux cubitus bien conservés ont été recueillis à la Lagoa dos Porcos. Le $\mathrm{n}^{\circ} 184229$ long de $433 \mathrm{~mm}$ et dont l'échancrure sigmoïde est haute de $78 \mathrm{~mm}$, appartient probablement à $P$. capivarae n. gen., n. sp. Du fait de ses dimensions bien supérieures (longueur $508 \mathrm{~mm}$, hauteur de l'échancrure sigmoïde $117 \mathrm{~mm}$ ) nous attribuons le spécimen $\mathrm{n}^{\circ} 188320$ à $T$. platensis.

\section{Métacarpien II}

Nous avons disposé d'un Mc II de $P$. capivarae n. gen., n. sp. de la Lagoa da Pedra (Conceição das Creoulas, Pernambuco), dont l'épiphyse proximale est endommagée. L'os est nettement plus court que celui de Toxodon platensis, avec une diaphyse moins profonde et une épiphyse distale plus petite, ses proportions sont donc bien différentes (Annexes, Tableau 7). 


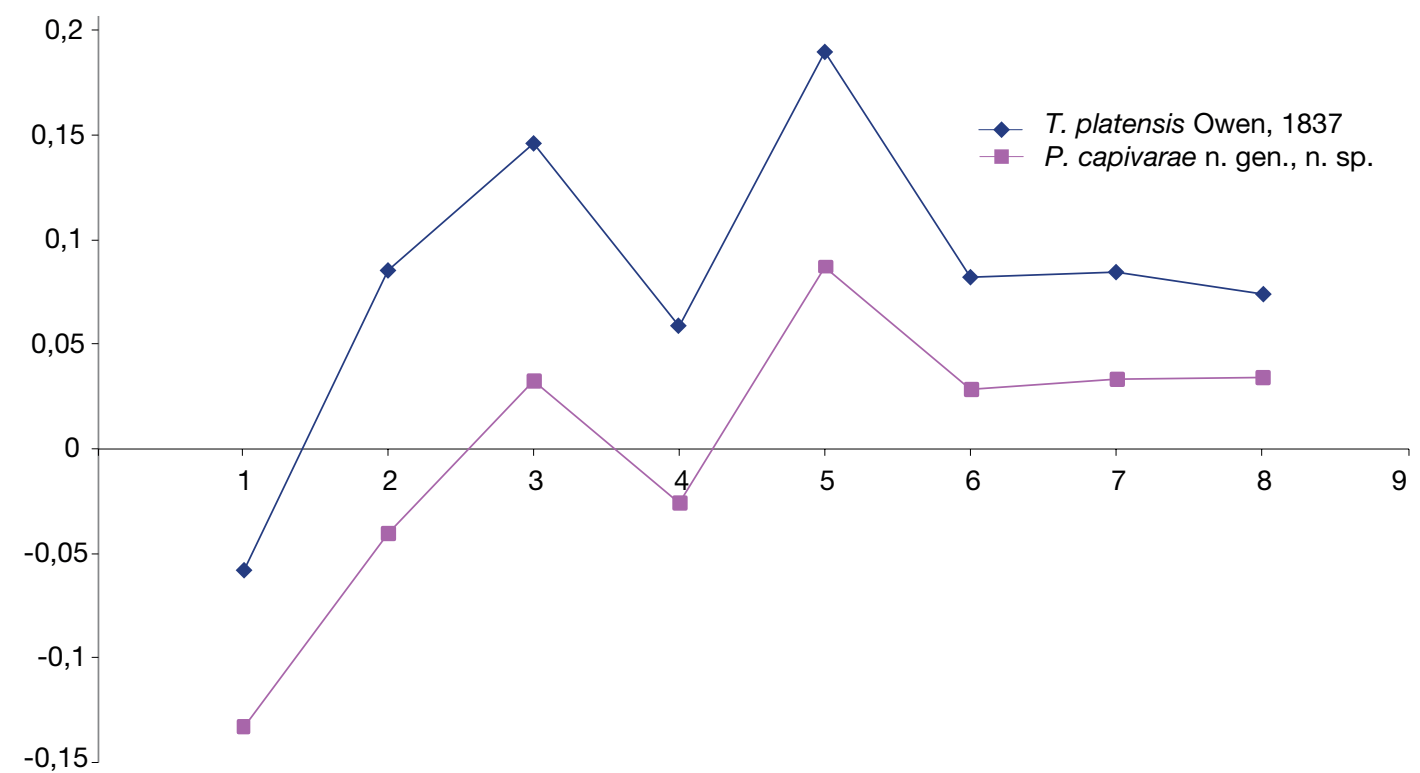

FIG. 12. - Diagramme des rapports du Mc III de Piauhytherium capivarae n. gen., n. sp., Lagoa do Quari, comparés aux valeurs moyennes des Mc III de Toxodon platensis Owen, 1837. Les variables numérotées en abscisse sont, dans l'ordre, celles indiquées Tableau 8. Le référentiel est Diceros bicornis (Linnaeus, 1758).

Nous connaissons trois spécimens de T. platensis, deux de Laje Grande à Pesqueira (fig. in Vidal 1959) et un de Bom Jardim dans le Pernambouco.

En vue de face la diaphyse apparaît sinueuse ; le bord proximal est oblique vers le bas et l'intérieur. La face proximale n'est pas articulaire dans sa partie la plus antérieure ; cette partie est suivie d'une facette articulaire triangulaire dont le sommet est disposé médialement. La face latérale porte une bande articulaire répondant au Mc III et étendue tout le long de l'épiphyse proximale.

Le Tableau 7 (Annexes) montre que les proportions moyennes des trois Mc II pernamboucains diffèrent peu de celles des valeurs minimales des Toxodon platensis d'Argentine : si leur longueur et le diamètre antéro-postérieur de la diaphyse sont du même ordre, leurs épiphyses sont toutefois moins volumineuses et leur diaphyse est plus étroite. Nous les rapportons donc à T. platensis.

Parmi plusieurs restes de Toxodontidae de la Fazenda Elefante à Gararu (Sergipe), Dantas et al. (2005: fig. 7c) décrivent un Mc II de façon suffisamment précise pour qu'il soit possible de l'attribuer à l'espèce T. platensis.

\section{Métacarpien III}

Le Mc III gauche nº 109005 de Quari (Fig. 11) est très bien conservé sauf pour les parties les plus postérieures de ses deux épiphyses, qui montrent de faibles traces de roulage.

L'articulation proximale, presqu'aussi large que longue, est en trapèze dont la grande base, disposée en avant, est légèrement déprimée.

En vue de face le contour du métacarpien médian rappelle beaucoup ceux des Rhinocerotidae et Hippopotamidae. La face antérieure s'élargit proximalement vers l'extérieur. Sa surface comporte une vaste dépression profonde étendue surtout côté médial et située un peu en dessous du bord proximal ; en dessous de la dépression la face antérieure reste concave transversalement ; vers son extrémité distale elle s'élargit fortement pour donner deux tubercules sus-articulaires, le latéral plus saillant et placé plus haut que le médial. La limite antérieure de l'articulation distale est bien visible, convexe vers le haut, légèrement assymétrique.

La face latérale s'élargit fortement vers le haut et se termine proximalement par deux bandes articulaires 

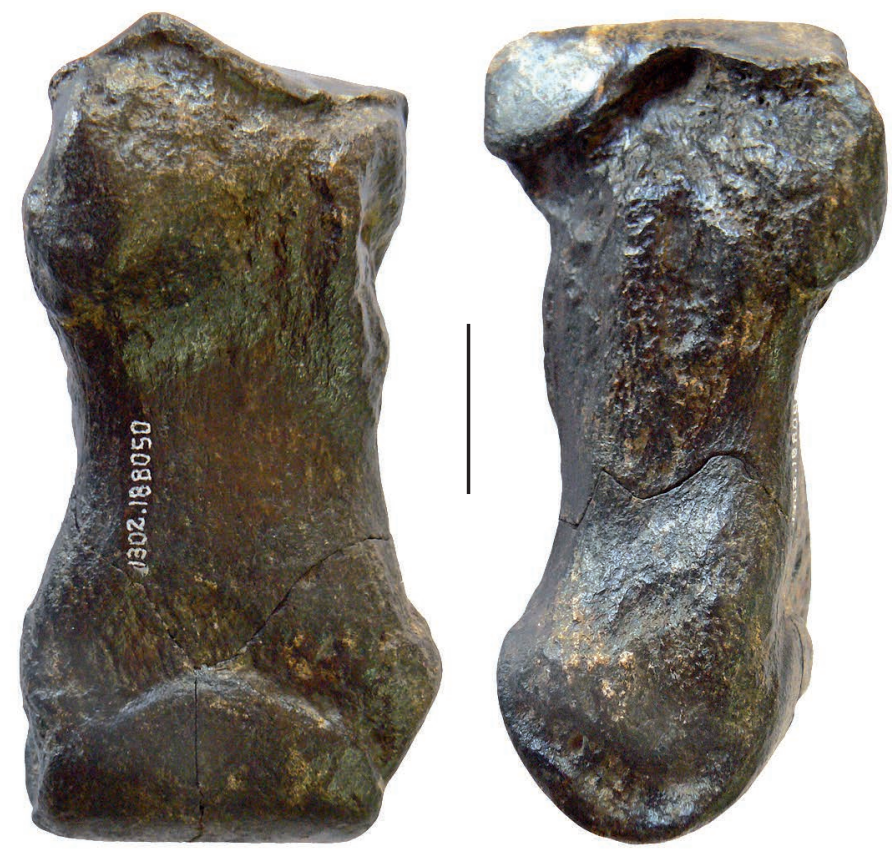

FIG. 13. - Piauhytherium capivarae n. gen., n. sp., Lagoa dos Porcos, Mc IV gauche $n^{\circ} 188050$ : A, vue de face; B, vue médiale. Échelle : $3 \mathrm{~cm}$.

continues, superposées, faisant entre elles un dièdre presque droit. La bande inférieure, qui répond au Mc IV, a son bord inférieur largement échancré, elle est bien plus haute en arrière qu'en avant et est bordée en dessous de sa moitié postérieure par un trou profond. La face médiale comporte une bande articulaire proximale aussi longue que l'épiphyse mais plutôt basse.

La section de la diaphyse est plus ou moins pentagonale, déprimée en avant, épaisse latéralement, plus mince et arrondie côté médial, avec une carène médiane épaisse sur sa face postérieure. L'articulation distale possède une petite quille-guide, épaisse et mousse, au milieu de sa face postérieure.

Les dimensions (Annexes, Tableau 8) sont toutes largement inférieures aux valeurs moyennes relevées sur un échantillon de 4 à 6 Toxodon platensis : elles sont de 12 à $26,4 \%$ plus petites que la moyenne de cet échantillon. Les proportions ne sont pas très différentes de celles correspondant aux valeurs minimales de ce dernier (Fig. 12) mais le Mc III de Piauhytherium n. gen. est nettement plus court, avec une diaphyse et une épiphyse distale plus larges.

Nous avons étudié un métacarpien médian de T. platensis de Santa Vitória do Palmar (Rio Grande do Sul, figuré in Souza Cunha [1959: pl. IV, fig. 1], conservé au Museu nacional), qui ne diffère en rien des spécimens argentins dont il a la taille et les proportions. Il en est de même pour le spécimen $\mathrm{n}^{\circ} 186530$ de la Lagoa dos Porcos, entier mais mal conservé et qui appartient à la même espèce.

\section{Métacarpien IV}

Deux Mc IV de P. capivarae n. gen., n. sp. proviennent de la Lagoa dos Porcos (Fig. 13). Deux autres conservés respectivement au Musée national de Rio et au Musée Câmara Cascudo de Natal proviennent de la Fazenda Vista Alegre à Taperoá $(\mathrm{PB})$ et de Lagoa do Santo à Currais Novos (RN).

D'autre part nous avons étudié sept Mc IV de T. platensis originaires du Nordeste, un de la Toca da Barra do Antonião, et six des Collections du 


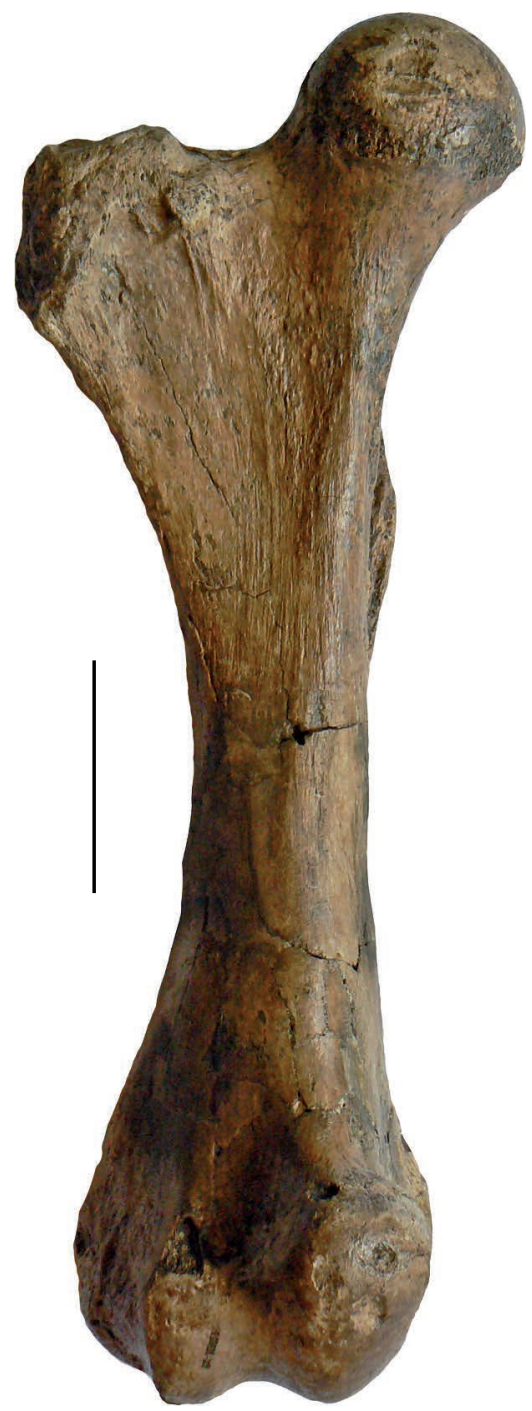

FIG. 14. - Piauhytherium capivarae n. gen., n. sp., Lagoa do Quari, fémur droit $n^{\circ} 108878$, vue antérieure. Échelle : $10 \mathrm{~cm}$.

Museu nacional. Parmi ces derniers, cinq sont de Laje Grande à Pesqueira (trois d'entre eux ont été figurés par Vidal en 1959), et un dernier spécimen, sans provenance connue, présente la même patine et les mêmes dimensions et proportions que les autres. Leurs dimensions sont assez proches des valeurs minimales des $T$. platensis argentins. Il en est de même pour les proportions, mais notre échantillon est limité à trois spécimens.
En vue de face le Mc IV est rétréci dans sa partie médiane et très élargi à ses extrémités. L'épiphyse proximale possède un bord supérieur rectiligne, oblique vers le bas et l'extérieur. La face antérieure est oblique vers l'extérieur, elle ne comporte pas de dépression bien délimitée ; elle a son bord supérieur en V inversé, le sommet du $\mathrm{V}$ étant très décalé médialement, d'où un bord pour l'essentiel rectiligne et oblique vers le bas et l'extérieur. Distalement le métapode s'élargit pour porter deux tubercules sus-articulaires, le latéral étant plus saillant et placé plus haut que le médial.

La face supérieure est trapézoïdale et entièrement articulaire sauf pour son bord antérieur. La face médiale est épaisse, élargie vers le haut ; à son sommet deux facettes articulaires successives répondent au Mc III ; c'est par exemple le cas du spécimen $\mathrm{n}^{\circ} 17 \mathrm{~V}$ de Laje Grande à Pesqueira, alors que le $\mathrm{n}^{\circ} 187 \mathrm{~V}$ provenant du même gisement porte une bande articulaire continue mais resserrée en son milieu ; on trouve de même sur le spécimen de la Toca da Barra do Antonião une bande articulaire deux fois plus haute dans sa partie postérieure que vers l'avant, et étendue sur toute la longueur de l'épiphyse.

La face latérale est nettement plus mince; elle présente proximalement, au milieu de son bord supérieur, une petite facette articulaire elliptique allongée horizontalement et soulignée par deux dépressions successives. L'articulation distale possède une petite quille-guide sur sa partie postérieure.

La section de la diaphyse est triangulaire, avec un sommet arrondi côté latéral, et deux autres sommets encadrant le côté médial.

Les Mc IV de la Lagoa dos Porcos sont plus courts et bien moins puissants que ceux de T. platensis, avec des dimensions globalement inférieures, notamment pour le DT maximal distal (Annexes, Tableau 9). Son articulation proximale est moins large mais relativement plus longue. En haut de la diaphyse la tubérosité antéro-médiale est nettement plus volumineuse. L'articulation proximo-médiale est plus haute en avant. Le bord antéro-latéral de l'articulation distale est bien moins oblique. 


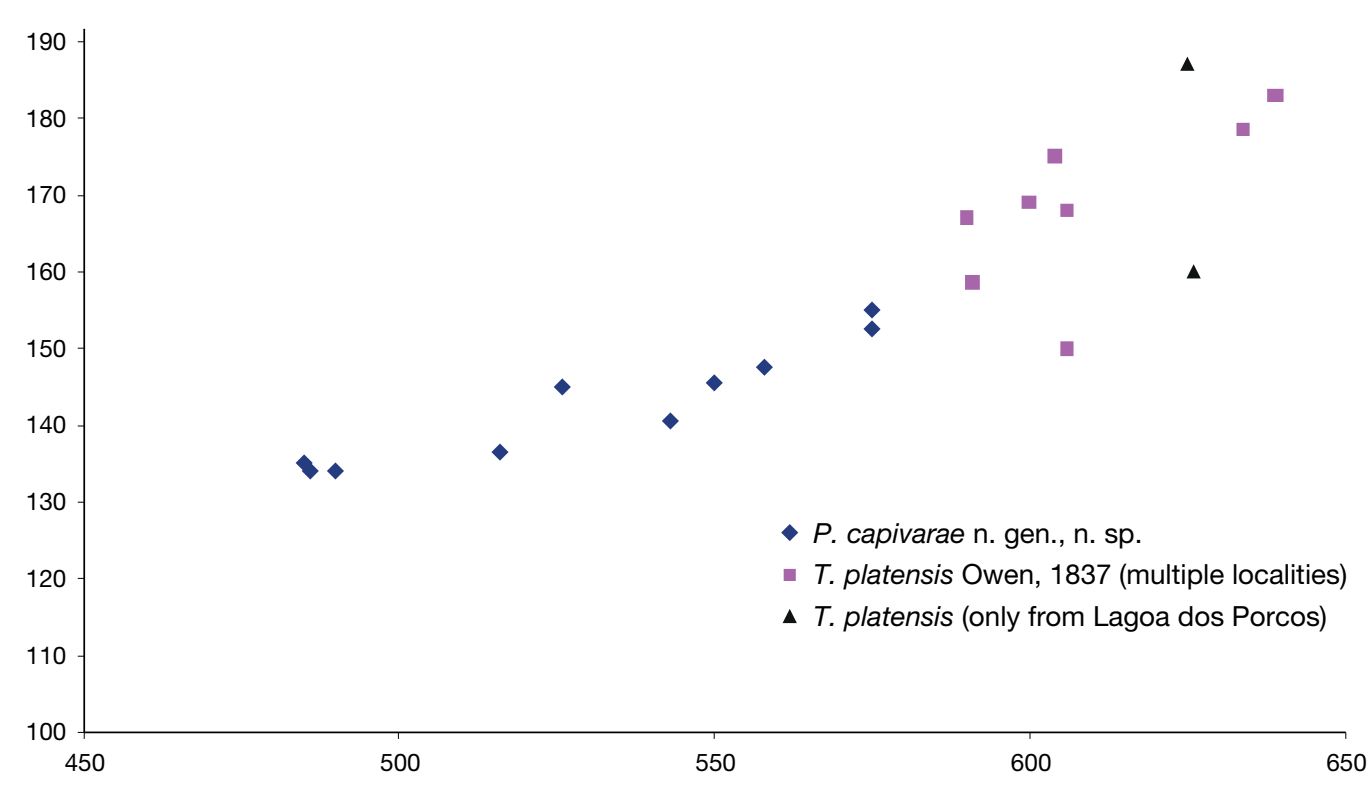

FIG. 15. - Diagramme de dispersion du DT distal en fonction de la longueur des fémurs de Piauhytherium capivarae n. gen., n. sp. et de Toxodon platensis Owen, 1837.

\section{Fémur}

C'est l'os long le mieux représenté, nous disposons de treize spécimens de $P$. capivarae n. gen., n. sp. (sept de la Lagoa dos Porcos et six de Quari). Nous en avons étudié aussi deux de la Lagoa da Cruz à Nova Cruz (Musée de Natal, RN). Au total neuf sont complets ou subcomplets.

L'os (Fig. 14) est très long, et sa tête, volumineuse et dépourvue de fovea capitis, est beaucoup plus haute que le grand trochanter, qui est irrégulier, bosselé et aplati à son sommet. Le col est long. À la jonction du col et de la face médiale de l'os s'élève un petit trochanter conique.

La diaphyse est longue, cylindrique, «prismaticorégulière" (Vidal 1959) et ne comporte pas de troisième trochanter. Elle est déprimée sur le tiers supérieur de sa face antérieure. Sur le tiers supérieur de sa face interne se trouve une lame en forme de trochanter. À la base de sa face externe existe un bourrelet vertical de 5 à $10 \mathrm{~cm}$ de long.

L'épiphyse distale volumineuse est surtout développée transversalement, le condyle externe est plus fort que l'interne, il porte une poulie articulaire très asymétrique dont le bord interne est large- ment avancé et très épais; en coupe transversale l'articulation distale fait un dièdre à peu près droit. En arrière de l'épiphyse distale le condyle interne est plus épais et moins oblique que le condyle externe. Shockey (2001) a observé qu'au niveau de l'articulation rotulienne, la crête de la trochée médiale est agrandie de la même façon que chez les chevaux, ce dispositif permettant de bloquer la rotule et les ligaments en position proximale. Un tel verrouillage de l'articulation implique que les Toxodon pouvaient rester debout pendant de longues périodes sans faire travailler leurs muscles extenseurs.

Nous avons comparé les fémurs de $P$. capivarae n. gen., n. sp. à un échantillon de T. platensis composé de 5 à 8 fémurs d'origine argentine, augmenté d'une épiphyse distale de la Lapa da Escrivania n ${ }^{\circ} 5$ (Lagoa Santa, Minas Gerais) du Musée de Copenhague, et d'un spécimen complet de Quixabinha (PE), figuré par Rolim en 1971 et 1974 (qui le rapportait avec doute à Trigodonops). À Lagoa dos Porcos, nous disposons aussi de deux très grands spécimens, pas très bien conservés mais cependant subcomplets. Le diagramme de 


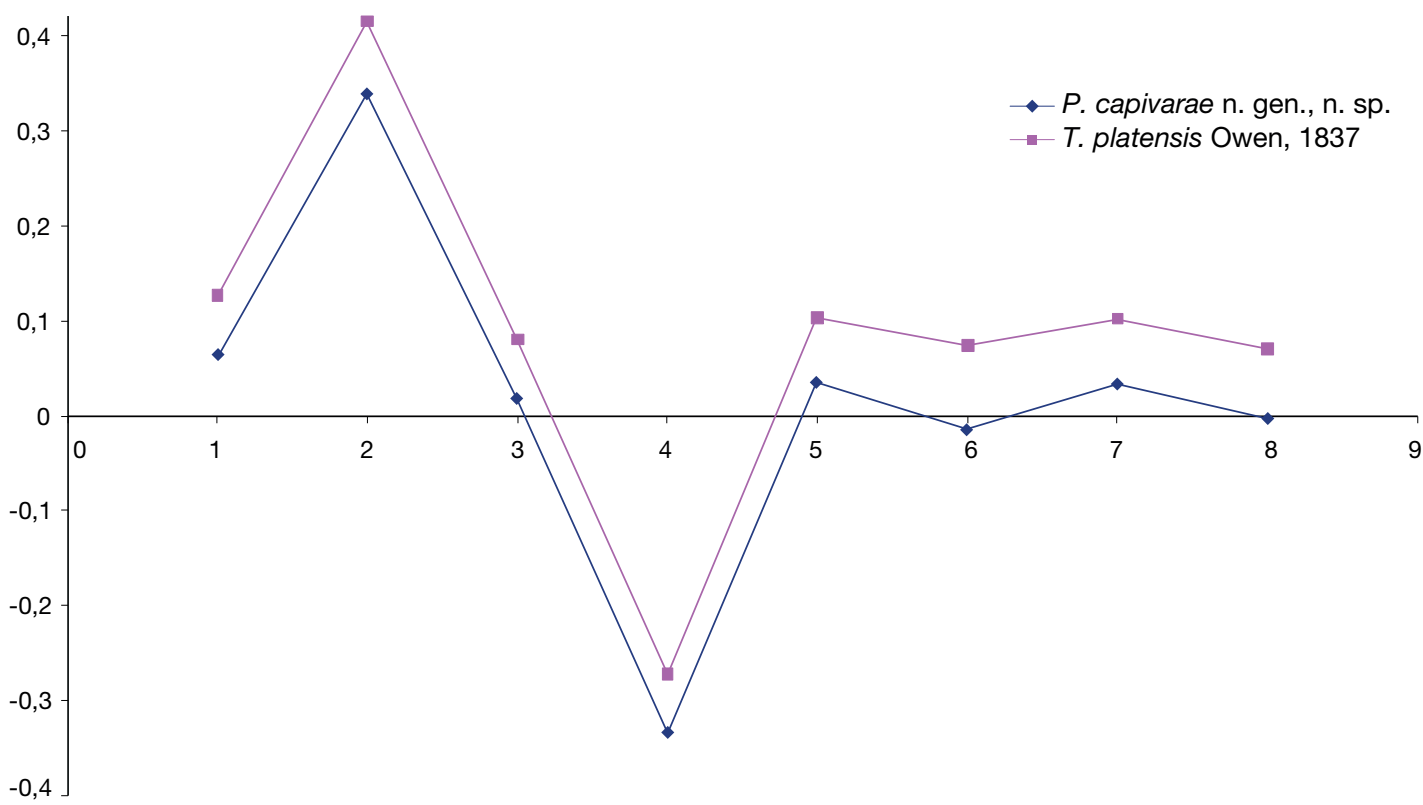

FIG. 16. - Diagramme des rapports des dimensions moyennes des fémurs de Piauhytherium capivarae n. gen., n. sp. comparés aux valeurs moyennes de Toxodon platensis Owen, 1837. Les variables numérotées en abscisse sont, dans l'ordre, celles indiquées Tableau 10. Le référentiel est Diceros bicornis (Linnaeus, 1758).

dispersion $\mathrm{L} \times \mathrm{DT}$ dist. de la Figure 15 montre que ces deux se placent parfaitement dans le nuage des T. platensis. qui ne montre aucune superposition avec celui des $P$. capivarae n. gen., n. sp. Les valeurs minimales de $T$. platensis, notamment la longueur (Annexes, Tableau 10), sont toujours un peu plus fortes que celles des plus grands $P i$ aubytherium n. gen. (Annexes, Tableau 10). Les proportions semblables pour l'épiphyse proximale diffèrent pour la diaphyse, moins profonde chez $P$. capivarae n. gen., n. sp., et l'épiphyse distale, moins large (Fig. 16).

\section{Tibia}

Nous disposons d'un tibia de la Lagoa dos Porcos associé à son péroné, tous deux bien conservés, et de deux tibias de Quari, dont un seul à peu près complet mais endommagé proximalement, déformé et plus ou moins écrasé. Les dimensions sont données en Annexes (Tableau 11). Elles sont le plus souvent (c'est notamment le cas pour la longueur) un peu inférieures aux valeurs minimales de notre échantillon de Toxodon platensis, lequel est composé de spécimens argentins auquel s'ajoute un tibia d'Urugaiana (Rio Grande do Sul) conservé au Museu nacional et qui leur est parfaitement semblable.

Le tibia des toxodontes est toujours très massif, mais sa diaphyse est très aplatie transversalement, presque tranchante, avec des épiphyses très larges (Figure 17) ; l'épiphyse distale ressemble à celle des Rhinocerotidae. Larticulation distale est oblique, l'apophyse stylö̈de (malléolaire interne) est plus prononcée que chez l'hippopotame et les rhinocéros (Gervais 1855), et le dessin de sa partie articulaire est différent.

Péroné et tibia sont totalement soudés proximalement et leur limite n'est pas visible. Le péroné est très élargi distalement, sa diaphyse à section triangulaire est tranchante en avant (Figure 17). Les DT totaux de l'ensemble tibia-péroné de la Lagoa dos Porcos sont de $143,5 \mathrm{~mm}$ pour le proximal et $136 \mathrm{~mm}$ pour le distal.

\section{Calcanéum}

L'os s'articule distalement avec le cuboïde, médialement avec l'astragale et le naviculaire. La facette 
pour le cuboïde se situe sur la face plantaire, à $90^{\circ}$ de sa position normale (Lavocat 1958 ; Paula Couto 1979). Van Frank (1957: 32-34, figs 11, 12) a décrit en détail les caractères du calcanéum de T. platensis qu'il oppose à ceux du calcanéum de San Miguel (Vénézuéla), appartenant très probablement à Mixotoxodon larensis.

Le spécimen de Quari est roulé et son sustentaculum tali est brisé. Celui de São Vitor est mieux conservé malgré une fracture entraînant l'absence de la tubérosité.

Le calcaneum se distingue bien de ceux de T. platensis par son sommet beaucoup moins étiré transversalement, sa diaphyse moins large à section plutôt triangulaire, et par son articulation antéro-distale : en reprenant la terminologie de van Frank (1957), la facette fibulaire est régulièrement convexe, sa limite avec la facette ectale est beaucoup moins nette; le sustentaculum à section plus triangulaire est plus développé, en vue postérieure il s'étend légèrement vers le bas alors que chez $T$. platensis il s'incline vers le haut. De ce point de vue les calcanéums de Quari et Sáo Vitor se rapprochent plus de celui de San Miguel ; en revanche les facettes cuboïdienne et navicularienne font entre elles un angle très obtus, et les facettes ectale et fibularienne sont de largeur voisine.

Le calcanéum gauche n 37016 de Garrincho est bien conservé, sa partie la plus distale est encore partiellement encroûtée dans la calcite. La taille est grande, la tête est beaucoup plus développée transversalement qu'antéropostérieurement, et il n'y a pas de véritable bec. Le sustentaculum tali est petit. Ce calcanéum présente tous les caractères de T. platensis: le corps de l'os a une section plutôt quadrangulaire ; en vue de face la facette fibulaire, faiblement concave transversalement, fait un fort dièdre avec la facette ectale ; cette dernière est plus étroite que la fibulaire; le sustentaculum montre une section quadrangulaire applatie et sa facette articulaire possède un contour subcirculaire. Les dimensions et les proportions correspondent à T. platensis (Annexes, Tableau 12).

Deux spécimens de Laje Grande à Pesqueira, dont un présentant de nombreuses exostoses latérales, un de Albardão à Santa Vitória do Palmar et deux autres présumés originaires de la région de São Paulo,

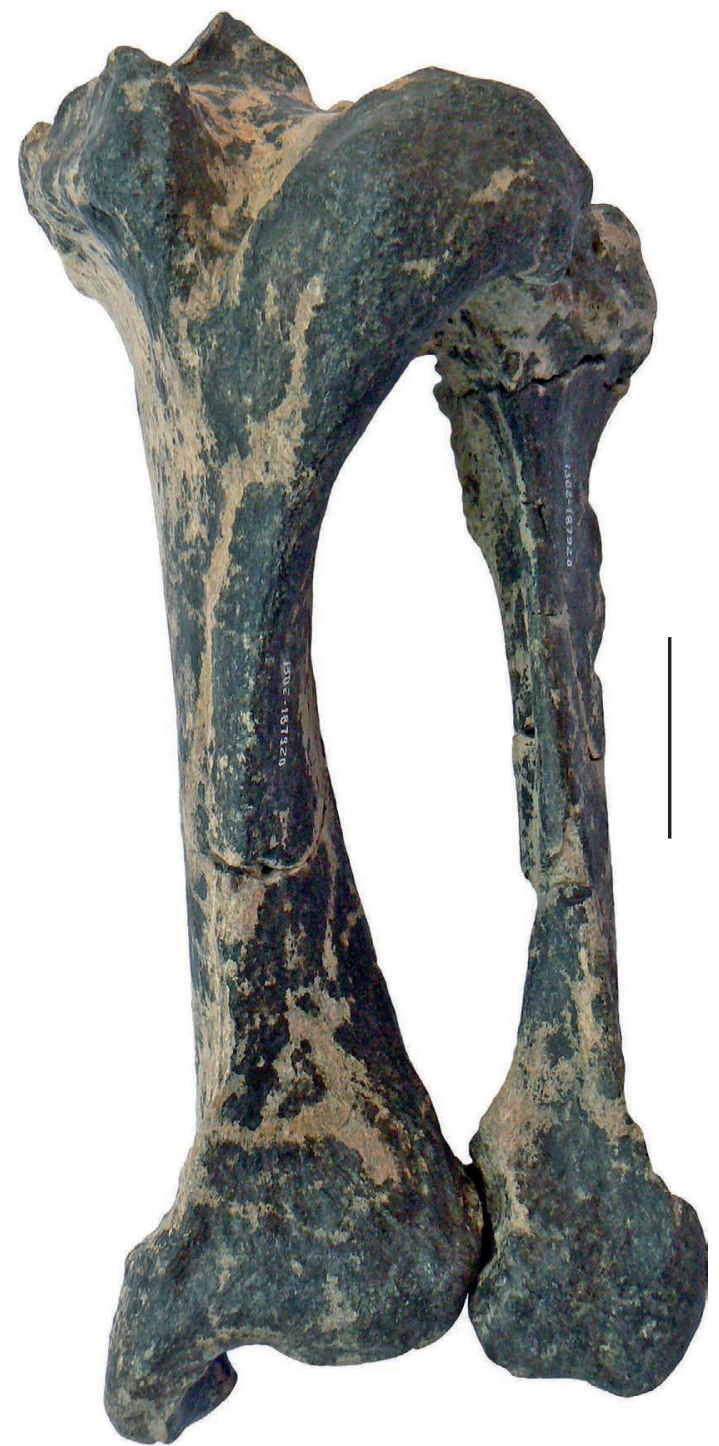

FIG. 17. - Piauhytherium capivarae n. gen., n. sp., Lagoa dos Porcos, tibia et péroné droits $n^{\circ} 187920$, vue antérieure. Échelle : $10 \mathrm{~cm}$.

conservés au Musée national de Rio de Janeiro, sont en tous points identiques aux calcanéums de T. platensis d'Argentine et à celui de Garrincho.

\section{AFFINITÉS}

Les restes de Piaubytherium capivarae n. gen., n. sp. présentent certains caractères anatomiques 


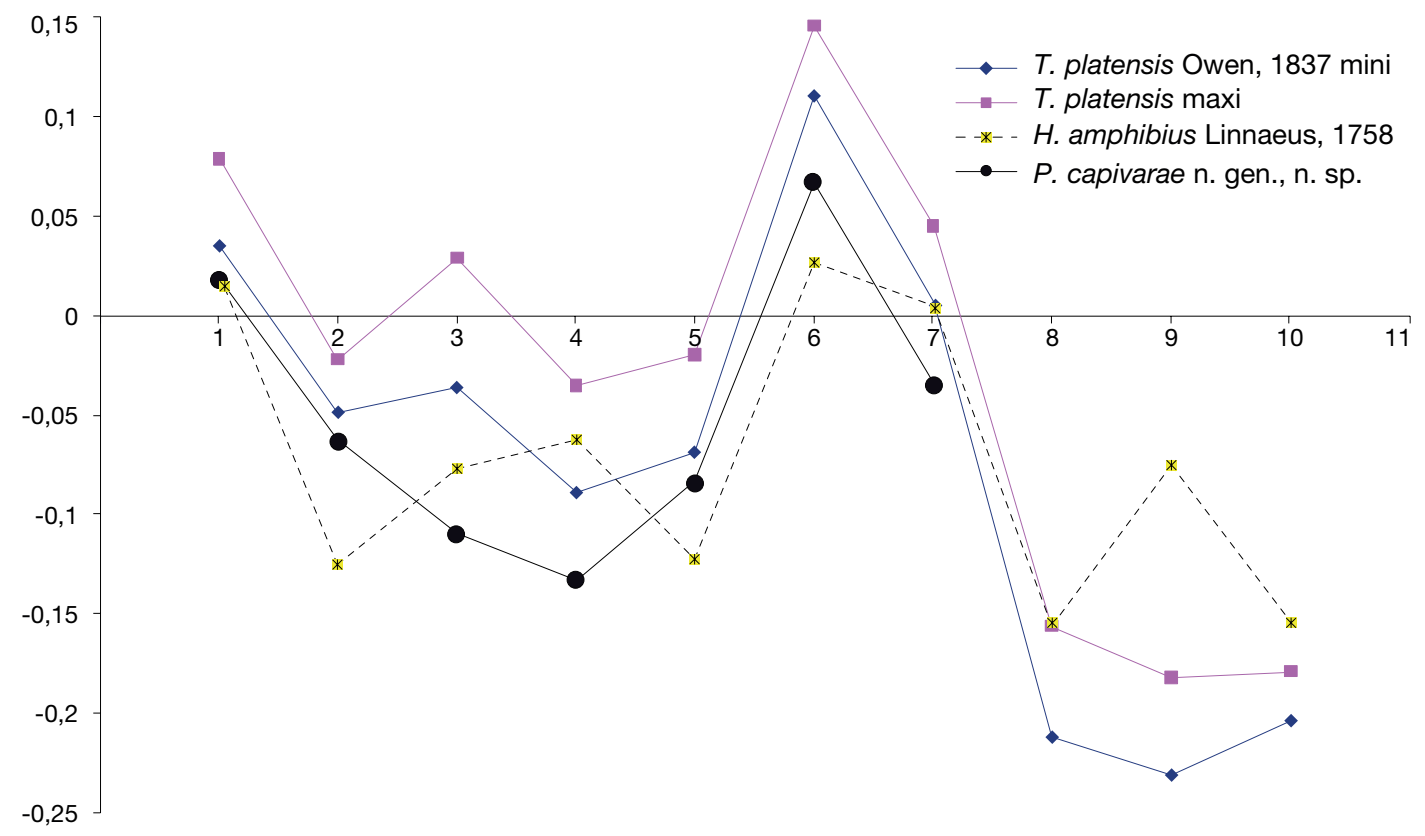

FIG. 18. - Diagramme des rapports des valeurs moyennes des segments de membres des Piauhytherium capivarae n. gen., n. sp., des valeurs extrêmes de Toxodon platensis Owen, 1837 et des valeurs moyennes de Hippopotamus amphibius Linnaeus, 1758 . Les variables numérotées en abscisse correspondent respectivement aux valeurs moyennes des longueurs de l'humérus, du radius, du Mc II, du Mc III, du Mc IV (Mc V pour I'hippopotame), du fémur, du tibia, du Mt II, du Mt III et du Mt IV (Mt V pour I'hippopotame). Le référentiel est Diceros bicornis (Linnaeus, 1758).

relevés chez Trigodonops et d'autres rappelant Mixotoxodon ou Toxodon. Ils ont aussi des caractères propres observables sur tous les spécimens disponibles provenant pourtant de gisements différents. Nasif et al. (2000) ont publié une analyse cladistique portant sur 20 genres de Toxodontidae et 54 caractères crâniens, mandibulaires et dentaires. Piauhytherium capivarae n. gen., n. sp. s'intègre mal dans leur cladogramme, car il passe mal le node 11 (caractère 53 , le pli ento-hypoconide des $\mathrm{M} / 3$ est peu profond mais bien marqué) et pas le node 12 (il y a un diastème derrière $\mathrm{I} / 3$, caractère 42 , et le pli paraconide-métaconide est bien marqué mais faible, caractère 48), ni les nodes en aval du 11 : (node 15, la $\mathrm{P} / 1$ est présente, caractère 43 , et node 16 , les prémaxillaires ne sont pas étendus latéralement, caractère 5). Ces caractères contribuent à faire de Piauhytherium n. gen. un genre nouveau.

\section{Genre Toxodon Owen, 1837}

Toxodon platensis - Souza Cunha 1959: 21-31, pl. IIVII. — Vidal 1959: 2-15, figs 4-7. — Guérin \& Faure 2008: 84, tableau.

Trigodonops lopesi-Rolim 1974b: 47, 76-79, figs 2.10, 2.27, 2.28, 2.29.

Toxodon sp. - Guérin 1991: 570. — Guérin et al. 1993a: 309, tableau 1, 323 ; 1993b: 194 ; 1996a: 63 tableau 1, 78. - Guidon et al. 1993: 486, 488. - Parenti et al. 1996: 346. — Peyre et al. 1998: 357.

\section{ESPÈCE TYPE. - Toxodon platensis Owen, 1837.}

Matériel ÉTUdié. - Dans les 14 sites brésiliens, dont 8 nordestins, où nous avons établi ou vérifié sa présence, Toxodon platensis est attesté par une cinquantaine de restes étudiables. Matériel étudié provenant de la région Nordeste : - Crâne et mandibule n ${ }^{\circ} 186001$ de la Lagoa dos Porcos, tous deux mal conservés.

- Deux rangées dentaires supérieures mal conservées du crâne n ${ }^{\circ} 186001$ et une M2/ n 186698 de la Lagoa 


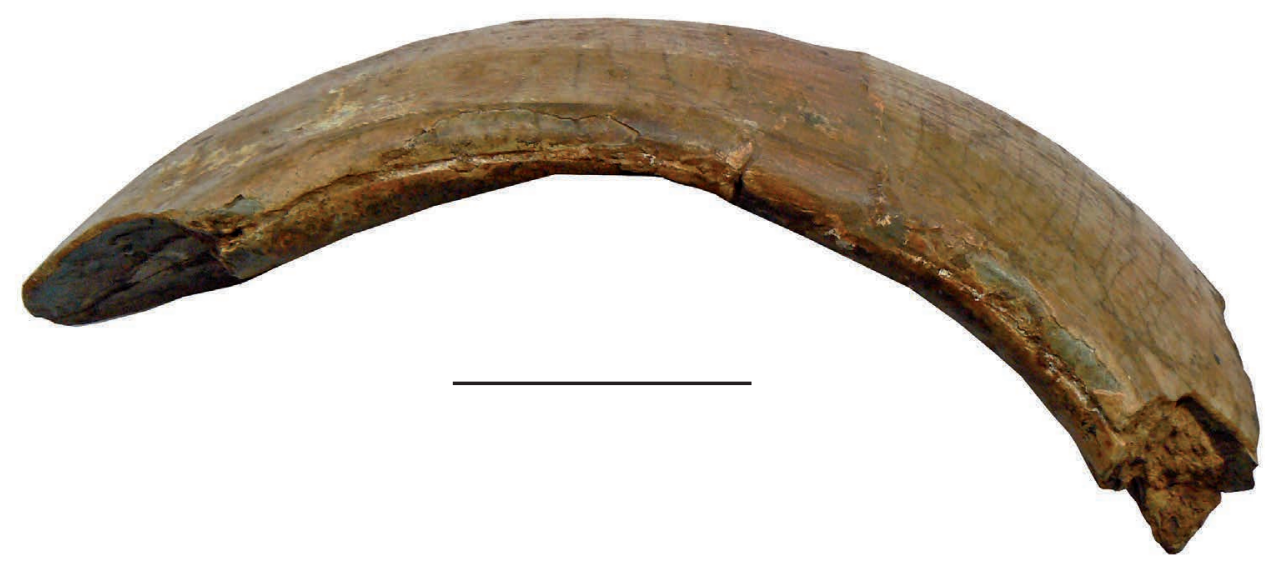

FIG. 19. - I2/ de Toxodon platensis Owen, 1837, Toca da Barra do Antonião, n 7373 . Échelle : $5 \mathrm{~cm}$.

dos Porcos ; quatre dents supérieures isolées de la Toca da Barra do Antonião (une P2/, deux P3/, une P4/) ; une M3/ de Lajedo da Escada à Baraúna (RN) (fig. in Damasceno 1973).

- Deux rangées dentaires inférieures mal conservées de la mandibule $\mathrm{n}^{\circ} 186001$ de la Lagoa dos Porcos ; quatre dents isolées de la Toca da Barra do Antonião (une $\mathrm{P} / 3$, une $M / 2$, deux $M / 3$ ) ; une $M / 2$ de Lajedo da Escada à Baraúna.

- Deux humérus de Laje Grande à Pesqueira et de Quixabinha à Petrolândia (PE).

- Un radius complet de Laje Grande et un incomplet de la Toca do Garrincho.

- Deux MC II de Laje Grande et un de Bom Jardim (PE).

- Un MC III de Lagoa dos Porcos n 186530.

- Cinq MC IV de Laje Grande.

- Deux fémurs de la Lagoa dos Porcos no 182086 et 182175 ; un de Quixabinha à Petrolândia.

- Un tibia incomplet de Bom Jardim.

- Astragale: un de Lajedo da Escada à Baraúna.

- Calcaneum: un de la Toca do Garrincho et trois de Laje Grande.

- Mt II : un de la Toca da Barra do Antonião et un de

la Fazenda Vista Alegre à Taperoá (PB).

- Mt III : un de la Toca da Barra do Antonião et un de Garrincho.

\section{GÉNÉRALITÉS}

L'espèce-type T. platensis est fondée sur des restes trouvés en 1833 par Darwin dans les Provinces de Santa Fe et d'Entre Rios en Argentine (Fernicola et al. 2009).

Toxodon platensis a été reconnu dans le CentreEst du Brésil dès les premières recherches de Lund dans la région de Lagoa Santa (Minas Gerais) pendant la première moitié du XIXe siècle (Lund 1950), mais cette trouvaille est restée longtemps inédite et n'a été publiée que beaucoup plus tard par Winge (1906). Paula Couto (1979), qui relate cela, note que le premier toxodonte signalé au Brésil l'a été en 1872 par Liais, qui décrit brièvement une M3/ d'une grotte de la Fazenda de Mocamba au Minas Gerais (Liais 1872: 420). Le premier toxodonte brésilien décrit scientifiquement l'a été en 1885 par Cope ; il s'agissait de restes provenant du Bahia, qui furent baptisés Toxodon expansidens Cope, 1885, taxon qui se révéla ultérieurement synonyme récent de T. platensis. Cette dernière espèce, reconnue par Holland en 1920 sous le nom de T. burmeisteri Giebel, 1866 à la Lagoa da Pedra Vermelha (Rio das Cantas, Bahia), a ensuite été trouvée dans le Rio Grande do Sul (Paula Couto 1942, 1944, 1978 ; Souza Cunha 1959). Récemment il a été identifié dans un site de São Bento do Una au Pernambuco (Mendonça 2007).

Toxodon platensis est attesté dès le Pléistocène moyen en Bolivie et devient abondant au Pléistocène supérieur. Récemment, des datations ESR ont été obtenues sur des restes de T. platensis du Pléistocène du Sud du Brésil (Lopes et al. 2010). Selon Bond et al. (1995), les derniers restes connus de Toxodon proviennent de la Formation La Postrera (province de Buenos Aires), datée du Lujanien supérieur 
(environ 8500 ans $\mathrm{BP}$ ), où ils seraient associés à des restes humains. Une prémolaire de Toxodon de Ponta de Flecha à Ribeira di Iguape, dans l'État de São Paulo, a été datée par ESR d'environ 6500 ans BP (Baffa et al. 2000), mais de nouvelles datations par AMS, donnant des dates comprises entre 12900 et 13770 en années calibrées BP, ont remis en cause ce résultat et considérablement vieilli ce spécimen (Neves et al. 2007).

La taille de $T$. platensis est au moins celle d'un rhinocéros ou d'un hippopotame du Nil, sa masse maximale était de l'ordre de 2 tonnes et plus; l'arrière-train est surélevé du fait du raccourcissement du membre antérieur. Pérez Garcia (2000) reconnaît deux morphotypes pour les incisives inférieures : l'un avec une section transversale comportant une large bande d'émail linguale (T. platensis), l'autre (T. burmeisteri, longtemps considéré comme une espèce à part entière) avec cette même bande présente mais étroite. En 1979 Paula Couto, rappelant que les deux espèces se distinguaient par les largeurs relatives des $\mathrm{I} 1 /, \mathrm{I} 2 /$ et $\mathrm{I} / 3$, avait déjà affirmé que T. burmeisteri ne se situait pas au delà de la variabilité individuelle ou sexuelle de T. platensis. Miño Boilini et al. (2006), apparemment d'accord avec l'idée de Pérez Garcia, rappellent que Lydekker avait suggéré un dimorphisme sexuel, T. platensis correspondant au mâle et T. burmeisteri, plus petit, à la femelle.

\section{DESCRIPTION}

Les restes de T. platensis homologues de ceux de $P$. capivarae n. gen., n. sp. ont été décrits ci-dessus à titre de comparaison, nous ne reprendrons donc pas leur description ici.

\section{Radius et cubitus}

Décrivant un radius de $T$. platensis de Pesqueira (PE), Vidal (1959) note qu'il augmente de volume distalement, et que l'épiphyse proximale porte une articulation en cupule relativement petite qui répond à la partie externe de l'épiphyse distale de l'humérus. Vidal note aussi que sur la face externe de l'épiphyse distale s'étend vers le bas une forte apophyse styloïde.

Le seul reste de radius de T. platensis provenant de la région de São Raimundo Nonato est un tiers distal recueilli à la Toca do Garrincho. Nous avons par ailleurs étudié un radius complet provenant de Laje Grande à Pesqueira ( ${ }^{\circ} 15 \mathrm{~V}$ du Museu nacional).

Ces deux pièces ne se distinguent ni anatomiquement ni biométriquement de 3 à 4 spécimens argentins de Toxodon platensis (Annexes, Tableau 6). Sauf pour le DAP proximal qui est un peu plus fort, le radius de Laje Grande se place pour toutes ses dimensions dans l'intervalle de variation de ces derniers.

Le bord latéral au-dessus de l'épiphyse distale est fortement concave, caractère mis en évidence par van Frank (1957) chez Toxodon, par opposition à Mixotoxodon où ce bord est convexe ; le radius attribué (avec prudence) par van Frank à Mixotoxodon est par ailleurs un peu plus petit que ceux des Toxodon dont nous avons disposé.

Le cubitus est remarquablement long par rapport au radius, avec lequel il n'est jamais soudé. Son plus grand volume se situe proximalement, au contraire du radius. L'olécrâne vertical est très développé, l'échancrure sigmoïde est fortement courbée (Vidal 1959).

\section{Astragale}

En vue de face l'os est très large distalement et la poulie apparaît bien dissymétrique, avec une lèvre latérale nettement plus haute que la médiale, bien que les deux bords de la gorge aient une inclinaison voisine; en vue de profil cette lèvre latérale est peu développée d'avant en arrière. Dès 1855 Gervais notait que la facette répondant au naviculaire "est rejetée à la partie inférieure de l'os dont elle occupe obliquement la partie antérieure»; il y a donc trois facettes articulaires inférieures, les deux autres répondant au calcanéum.

Un spécimen de Lajedo da Escada à Baraúna (RN, Musée de Natal), et trois conservés au Musée national de Rio de Janeiro, un de Albardão, un du ruisseau Chuí, tous deux à Santa Vitória do Palmar (Rio Grande do Sul), et un autre présumé provenir de la région de Sáo Paulo, sont en tous points identiques aux astragales de T. platensis d'Argentine.

Les dimensions sont données en Annexes (Tableau 12), elles sont semblables pour les deux échantillons. 


\section{Métatarsien II}

Le métatarsien II droit n ${ }^{\circ} 1425$ de la Toca da Barra do Antoniāo est particulièrement bien conservé.

En vue de face l'os est rétréci au milieu et bien élargi au niveau des épiphyses. Le bord proximal de la face antérieure est fortement concave avec une apophyse à chaque extrémité. La face antérieure porte une gouttière de torsion oblique vers le bas et l'intérieur, bordée en son milieu et latéralement par une petite dépression. Distalement apparaissent deux tubercules sus-articulaires, le latéral étant plus saillant et placé plus haut que le médial.

La face supérieure est en partie occupée par une vaste facette articulaire aux bords antérieur et postérieur convexes, au bord médial à peu près droit et au bord latéral concave ; ce bord latéral fait un dièdre obtus avec la facette articulaire du sommet de la face latérale ; cette articulation proximale répond à l'ensemble soudé constitué par le petit cunéiforme et le cunéiforme I. La partie antérieure de la face proximale n'est pas articulaire ; les deux apophyses verticales dont nous parlions à propos de la vue de face s'y trouvent dans l'alignement des bords latéral et médial de l'articulation.

La face latérale est très épaissie vers le haut; à son bord proximal elle porte une longue facette articulaire répondant au Mt III, et, pour sa partie supérieure, au grand cunéiforme. La face médiale est plus mince ; son sommet est à pan coupé, dessinant ainsi un triangle orienté pointe en bas ; ce triangle n'est pas articulaire. La face postérieure est déprimée transversalement, son bord latéral est une épaisse crête mousse.

La section de la diaphyse est complexe, très épaisse latéralement.

Les dimensions (Annexes, Tableau 14) sont proches des valeurs minimales relevées sur un échantillon de 4 à 5 Toxodon platensis d'Argentine, elles sont même souvent inférieures à ces valeurs; elles sont voisines de celles d'un spécimen de Taperoá (PB) et d'un autre du ruisseau Chuí (RS), tous deux conservés au Museu nacional de Rio de Janeiro, et que nous attribuons à cette même espèce. Rappelons que Bergqvist et al. (1997), hésitant entre T. platensis, Trigodonops lopesi et même Mixotoxodon larensis, avaient désignés les restes de Taperoá Toxodontidae indét.

\section{Métatarsien III}

Le Mt III gauche nº5355 de la Toca da Barra do Antoniāo est roulé, et la partie postérieure de son épiphyse proximale n'est pas totalement conservée. Le Mt III gauche n47734 de la Toca do Garrincho, par ailleurs plus grand, est au contraire en très bon état.

L'os est court, plat et large, d'allure éléphantoïde. La face supérieure est totalement articulaire, en triangle dont le plus grand côté est le bord antérieur ; cette articulation répond au grand cunéiforme.

La face antérieure est fortement déprimée en son milieu. Son bord supérieur est sub-rectiligne, presque horizontal. Elle est très élargie distalement par deux puissants tubercules sus-articulaires, le latéral étant plus développé et situé plus bas que le médial. Sur cette face la limite antérieure de l'articulation distale est bien marquée, très dissymétrique.

La face latérale très épaissie proximalement porte à son bord supérieur deux facettes articulaires ; l'antérieure, beaucoup plus vaste, est concave verticalement sur le spécimen de la Toca do Garrincho ; elle est plutôt plane sur le métapode de la Toca da Barra do Antonião.

La face médiale porte proximalement une petite bande articulaire très basse, dont la surface est mal individualisée.

La section médiane de la diaphyse est trapézoïdale, avec la grande base en avant.

Les dimensions (Annexes, Tableau 15) et les proportions des deux spécimens nordestins correspondent bien à Toxodon platensis.

\section{AFFINITÉS}

Les T. platensis du Rio Grande do Sul (Albardão et ruisseau Chuí à Santa Vitória do Palmar, Uruguaiana), de la région de São Paulo et de Lagoa Santa (MG), ne se distinguent ni morphologiquement ni biométriquement de leurs homologues d'Argentine. En revanche, parmi ceux de la région Nordeste (Toca da Barra do Antonião, Toca do Garrincho [PI], Bom Jardim, Laje Grande à Pesqueira, Quixabinha [PE], Caverno Lajedo da Escada à Baraúna [RN], Fazenda Vista Alegre à Taperoá [PB]), la plupart, s'ils en conservent les proportions, sont en général un peu plus petits et assez souvent moins trapus, c'est notamment le cas pour les métacarpiens et les 
métatarsiens. Nous avions observé un phénomène analogue chez Macrauchenia patachonica Owen, 1840 (Guérin \& Faure 2004). S'agit-il, dans un cas comme dans l'autre, d'une population différente, relevant d'une sous-espèce géographique particulière, ou plus simplement d'un effet de la Loi de Bergmann entraînant une augmentation ou une réduction de taille des individus selon un gradient latitudinal ? Seules de nouvelles découvertes permettront de trancher entre ces deux hypothèses.

\section{DISCUSSION PALÉOÉCOLOGIQUE}

Tous les Toxodontidés pléistocènes, avec leur port de tête bas et leurs jugales hyperhypsodontes étaient, quelle que soit leur espèce, parfaitement adaptés pour tondre les graminées. Il est toutefois probable que les Toxodon, au muffle large, n'avaient pas exactement les mêmes exigences que Mixotoxodon et Piauhytherium n. gen. dont le museau était rétréci en avant. Van Frank (1957: 27) suggère pour Toxodon un régime à base de plantes de marécages et de racines, analogue à celui de l'actuel Rhinoceros unicornis (qui est pourtant beaucoup moins hypsodonte), et, pour Mixotoxodon, "the character of our Recent grazers and tree-browsers» sans consommation de racines. MacFadden a présenté en 2004 les résultats d'une analyse des isotopes du carbone réalisée sur des dents de Toxodon et de Mixotoxodon provenant de 13 gisements de basse altitude et datant de 38500 à 5517 années BP (MacFadden 2004) ; ils montrent une grande diversité de régimes alimentaires, fondés soit sur des végétaux en C3, soit sur des végétaux en $\mathrm{C} 4$, soit encore sur un mélange des deux, les biotopes correspondants variant de la forêt à la prairie.

Melo et al. (2007) ont mis en évidence des cas d'hypoplasie de l'émail sur des dents de Toxodon du Pernambouco (Laje Grande à Pesqueira), de la région de São Paulo et du Rio Grande do Sul (Santa Vitória do Palmar). Les spécimens du Sud du Brésil présentent les cas les plus nombreux, ce que les auteurs interprètent comme le résultat d'un stress qui pourrait être lié au maximum glaciaire.

Selon les divers auteurs, ces animaux avaient des moeurs affines de celles des rhinocéros et/ou des hippopotames actuels, ce qui implique dans la deuxième hypothèse une plus ou moins grande proximité de l'eau, voire des moeurs amphibies ; la première hypothèse laisse subsister quant à elle un spectre de probabilités plus étendu, les cinq espèces de rhinocéros actuelles ayant des biotopes préférentiels bien différents, aucune toutefois n'étant aquaphile, bien que nombre d'espèces fossiles l'aient été (Prothero et al. 1989). Cependant le graphe des rapports de segments de membres (Fig. 18), qui prend en compte les longueurs de dix os, met en évidence trois faits :

- 1) par rapport à un grand rhinocéros peu spécialisé comme Diceros bicornis, Toxodon platensis se caractérise par la grande longueur du segment I de la patte antérieure (humérus) et surtout de la patte postérieure (fémur), un segment II très raccourci, surtout à l'avant (radius), un segment III relativement court à l'avant (métacarpiens) et très court à l'arrière (métatarsiens).

- 2) Toxodon platensis présente beaucoup plus de similitudes avec Hippopotamus amphibius Linnaeus, 1758 (pour lequel n'a été pris en compte que le plus long des deux métapodes axiaux, à savoir le troisième) qu'avec le rhinocéros, montrant que son mode de vie était bien plus proche de celui de l'hippopotame (données biométriques in Faure [1985]).

- 3) Piaubytherium capivarae n. gen., n. sp. n'est représenté que par sept os sur les dix utilisés dans le diagramme (la longueur du radius a été évaluée à partir de celle du cubitus). Les longueurs absolues sont toujours nettement plus faibles que chez T. platensis, mais les rapports de ces longueurs entre elles sont du même ordre, traduisant un mode de vie analogue.

On remarquera que les sites à Toxodontidae qui ont livré des tapirs sont très rares, à notre connaissance seul Lajedo de Escada au Rio Grande do Norte (Porpino \& Santos 2003) est signalé dans le Nordeste, ce qui montre surtout que les Toxodontes ne vivaient pas en forêt dense, fût-elle marécageuse. Dans les gisements, et notamment les Lagoas du Nordeste, les Toxodontidae sont communément associés à des restes d'Eremotherium Spillmann, 1948, de Catonyx Ameghino, 1891, Scelidodon Ameghino, 1891, Glyptodon Owen, 1839, Panochthus Burmeister, 1866, Pampatherium Ameghino, 1875. 
Haplomastodon Hoffstetter, 1950, Macrauchenia Owen, 1840, Hippidion Owen, 1859, Equus Linnaeus, $1758 \ldots$ Â noter que petits mammiferes et prédateurs ne se retrouvent pratiquement jamais dans les lagoas, ces absences s'expliquent probablement par un tri gravimétrique et granulométrique, dû aux circulations hydrauliques.

Grands, lourds et très puissants, dotés de quatre défenses (les I2/ et I/3) longues, larges, aigües et tranchantes, à croissance continue, véritables fers de lances constituant sans aucun doute des armes très efficaces (voir par exemple la I $2 / \mathrm{n}^{\circ} 7373$ de la Toca da Barra do Antonião, Fig. 19), les Toxodontidés du Pléistocène supérieur n'avaient probablement pas de prédateurs à l'âge adulte : les deux grands Carnivores qui étaient alors au sommet de la pyramide alimentaire, Smilodon populator (Lund, 1840) et Arctotherium bonariense (Gervais, 1852), étaient impuissants contre eux.

Beltrão et ses co-signataires sont les seuls, à notre connaissance, à avoir reconnu une scène de chasse au toxodon dans l'art rupestre du Nordeste brésilien. Il s'agit d'une peinture à l'ocre rouge du site Lagerdão à Riacho Largo (Central, Bahia) dont le relevé a été publié à de nombreuses reprises (Bigarella et al. 1984 ; Beltrão \& Locks 1990: 106, fig. 8 ; Beltrão 2000 : 105, 129, fig. 55 ; 2008: 137, 168). D'après ces illustrations le quadrupède représenté est méconnaissable. Comme nous l'avons déjà remarqué par ailleurs (Faure et al. 2010), dans l'état actuel des connaissances, l'art rupestre du Nordeste brésilien ne comporte pas de figuration de la mégafaune disparue à la fin du Pléistocène supérieur, l'attribution de Beltrão serait intéressante si elle était plus convaincante.

\section{CONCLUSION}

Les travaux de fouilles effectuées dans les sites paléontologiques de la région de São Raimundo Nonato (SE du Piauí), et notamment dans les Lagoas, ont permis la découverte de nombreux restes de mégafaune, souvent bien conservés. Parmi ces fossiles nous avons pu reconnaître deux genres de Toxodontidae dans le Pléistocène supérieur du Brésil intertropical, Toxodon et le genre nouveau Piauhytherium n. gen., dont les exigences écologiques étaient probablement différentes. Toxodon platensis est attesté dans huit gisements nordestins (nous avons étudié l'espèce dans 14 sites brésiliens au total), et la population de la Région Nordeste montre quelques particularités que de nouvelles découvertes permettront très probablement d'interpréter. Piauhytherium capivarae n. gen., n. sp. est reconnu dans quatre gisements, tous de la Région Nordeste. Leur sympatrie est vérifiée dans deux sites : la Toca do Garrincho et la Lagoa dos Porcos.

\section{Remerciements}

Nous tenons à remercier Niède Guidon et AnneMarie Pessis de la Fundação Museu do Homem Americano (FUMDHAM), de nous avoir confié l'étude paléontologique des sites de la région du Parc National Serra da Capivara, et pour toutes les facilités qu'elles nous ont offertes lors de nos diverses missions à São Raimundo Nonato (PI). Nous remercions aussi nos collègues et ami(e)s Fabio Parenti, Gisele Daltrini Felice, Maria de Fátima da Luz, Marcela Pacini Valls et leurs équipes de fouilles pour le travail effectué dans la Lagoa do Quari, et la Lagoa dos Porcos, ainsi que l'équipe technique du laboratoire de Paléontologie, en particulier Simone Belarmino da Silva, qui a réalisé entre autres la restauration du crâne provenant de la Lagoa São Vitor, Iderlan de Souza, Niède da Silva Dias, Valdeci da Silva Paes et Leandro Santos Paes. Le résumé en portugais est dû à Maria de Fátima da Luz.

Nous devons à l'obligeance de A. W. A. Kellner, responsable de la Section Paléovertébrés, d'avoir pu accéder aux collections du Museu Nacional de Rio de Janeiro ; Gabriela Martin Avila et Marcos Galindo Lima de l'UFPE pour le matériel de la Lagoa da Pedra à Conceição das Creoulas (Pernambouco), C. Aguilar et M. de F. C. F. dos Santos, du Museu Câmara Cascudo de Natal, pour celui du Rio Grande do Norte ; et G.S. Montalverne Girão, Directrice du Museu diocesano Dom José de Sobral.

En Europe, nous avons pu travailler sur les Toxodontidae conservés dans les collections du Muséum national d'Histoire naturelle à Paris grâce à nos collègues parisiens, notamment Christian de Muizon; nous remercions également Andy 
Currant qui nous a donné accès à la collection de mammifères sud-américains du Natural History Museum à Londres ; Tove Hatting et Knud Rosenlund pour celle du Zoologisk Museum à Copenhague ; Louis Chaix et Jacqueline Studer ont mis à notre disposition les fossiles du Muséum d'Histoire naturelle de Genève.

Nous souhaitons enfin remercier Esperanza Cerdeño Serrano et Annemarie Ohler, qui nous ont permis d'améliorer une première version de ce manuscrit.

Nos missions au Brésil ont été financées par la Fundação Museu do Homem Americano (FUMDHAM), le Conselho Nacional de Desenvolvimento Cientifico e Tecnológico (CNPq), l'Instituto $\mathrm{Na}$ cional de Arqueologia, Paleontologia e Ambiento do Semi-Árido (INCT/CNPq), la Fundação Seridó (qui a pris en charge notre déplacement dans le Rio Grande do Norte), et la Petróleo Brasileiro SA-Petrobras.

\section{RÉFÉRENCES}

Baffa O., Brunetti A., Karmann I. \& Martins Dias NETO C. 2000. - ESR dating of a Toxodon tooth from a Brazilian karstic cave. Aplied Radiation and Isotopes 52: 1345-1349.

Beltrão de Moraes Coutinho M. C. 2000. - Ensaio de Arqueogeologia, uma abordagem transdisciplinar. ZIT Grafica e Editora, Rio de Janeiro, 168 p.

Beltrão de Moraes Coutinho M. C. 2008. - Le peuplement de l'Amérique du Sud. Essai d'archéogéologie: une approche interdisciplinaire. Riveneuve, Paris, $221 \mathrm{p}$.

Beltrâo de Moraes Coutinho M. C. \& Locks M. 1990. - Climatic changes in the archaeological region of Central, Bahia, Brazil, as shown by interpretation of pre-historic rock paintings. Proceedings of the first international Congress of Ethnobiology "Ethnobiology: implications and applications", Belém, 1988: 99-112.

Bergqvist L. P., Gomide M., Cartelle C. \& Capilla R. 1997. - Faunas-locais de mamíferos pleitocênicos de Itapipoca/Ceará, Taperoá/Paraíba e Campina Grande/Paraíba. Estudo comparativo, bioestratinômico e paleoambiental. Revista universidade Guarulhos, Geociências 2 (6): 22-32.

Bigarella J. J., Beltrāo de Moraes Coutinho M. C. \& Moraes Rego Toth E. 1984. - Registro de fauna na arte rupestre : possiveis implicaçóes geologicas. Revista de Arqueologia 2 (1): 31-37.
Bond M., Cerdeño E. \& Lopez G. 1995. — Los Ungulados nativos de América del Sur, in Alberdi M. T., LEONE G. \& TonNi E. P. (éds), Evolucion biologica y climatica de la region pampeana durante los ultimos cinco millones de años, Museo Nacionales de Ciencias naturales édit., Madrid: 259-275.

Cartelle C. 1994. - Tempo passado. Mamiferos do Pleistoceno em Minas Gerais. Acesita, Belo Horizonte, $132 \mathrm{p}$.

Chaves S. A. M., Parenti F., Guérin C., Faure M., Candelato F., Rioda V., Mengoli D., Ferrari S., Natali L., Scardia G. \& Oberlin C. 2008. — Palinological analyses of Quarternary lacustrine sediments from "Lagoa do Quari”, NE Brazil, (PI). FUMDHAMentos VII: 63-68.

Cisneros J. C. 2005. - New Pleistocene vertebrate fauna from El Salvador. Revista brasileira de Paleontologia 8 (3): 239-255.

Damasceno J. M. 1973. - Ocorrência de Toxodon platensis Owen, 1840 em Olho d'Agua da Escada, distrito de Baraúna, Municipio de Mossoro, Rio Grande do Norte. Arquivos Instituto Antropologico "Câmara Cascudo", Paleont. 1: 1-18.

Dantas M. A. T. \& Tasso M. A. DE L. 2007. - Megafauna do Pleistoceno final de Vitória da Conquista, Bahia: taxonomia e aspectos tafonômicos. Scientia Plena 3 (3): 30-36.

Dantas M. A. T., Zucon M. H. \& Ribeiro A. M. 2005. - Megafauna pleistocênica da Fazenda Elefante, Gararu, Sergipe, Brasil. Geociências 24 (3) : 277-287.

FAURE M. 1985. - Les hippopotames quaternaires non insulaires d'Europe occidentale. Nouvelles Archives du Muséum d'Histoire naturelle de Lyon 23 : 13-79.

Faure M., Guérin C. \& Mourer-Chauviré C. 2010. L'art rupestre du Parc national Serra da Capivara, Piauí, (Brésil) : bestiaire figuré et données paléontologiques. XIV Congresso Internacional de Arte Rupestre IFRAO "Global Rock Art", São Raimundo Nonato 29 de junho-3 de julho 2009, FUMDHAMentos IX, vol. II: $1-15$.

Fernicola J. C., Vizcaíno S. F. \& De Iulis G. 2009. The fossil Mammals collected by Charles Darwin in South America during his travels on board the HMS Beagle. Revista de la Asociación Geológica Argentina, 64 (1): 147-159.

FerrusQuia-VIllafranca I. 1978. - XIII. Distribution of Cenozoic vertebrate faunas in Middle America and problems of migration between North and South America, in Ferrusquia-Villafranca I. (éd.), Conexiones terrestres entre Norte y Sudamérica. Universidad Nacional Autonoma de México, Instituto de Geologia, Vol. 101: 193-321.

FRANK R. VAN- 1957. - A fossil collection from Northern Venezuela. 1. Toxodontidae (Mammalia, Notoungulata). American Museum Novitates 1850: 1-38.

GERVAIS P. 1855. - Recherches sur les mammiferes fossiles de l'Amérique méridionale. P. Bertrand, 63 p. 
GuÉRIN C. 1980. — Les rhinocéros (Mammalia, Perissodactyla) du Miocène terminal au Pléistocène supérieur en Europe occidentale. Comparaison avec les espèces actuelles. Documents des Laboratoires de Géologie de Lyon, 79, 1185 p.

GUÉRIN C. 1991. — La faune de vertébrés du Pléistocène supérieur de l'aire archéologique de São Raimundo Nonato (Piauí, Brésil). Comptes Rendus de l'Académie des Sciences de Paris 312, II: 567-592.

GUÉRIN C. 1993. — La faune pléistocène de la Lagoa da Pedra à Conceição das Creoulas/Salgueiro, Pernambuco, Brésil. Clio, Recife, sér. Arqueológica 1, 9: 15-20.

Guérin C. \& Faure M. 2004. - Macrauchenia patachonica Owen (Mammalia, Litopterna) de la région de Sáo Raimundo Nonato (Piauí, Nordeste brésilien) et la diversité des Macraucheniidae pléistocènes. Geobios 37: 516-535.

GUÉRIN C. \& FAURE M. 2008. — La biodiversité mammalienne au Pléistocène supérieur - Holocène ancien dans la Région du Parc National Serra da Capivara (SE du Piauí, Brésil). FUMDHAMentos, São Raimundo Nonato, VII: 80-93.

Guérin C., Curvello M.A., Faure M., Hugueney M. \& Mourer-Chauviré C. 1993a. - La faune pléistocène du Piauí (Nordeste du Brésil) : implications paléoécologiques et biochronologiques. Quaternaria nova 3: 303-341.

Guérin C., Hugueney M., Mourer-Chauviré C. \& FAURE M. 1993b. - Paléoenvironnement pléistocène dans l'aire archéologique de São Raimundo Nonato (Piauí, Brésil) : apport des mammiferes et des oiseaux. Documents des laboratoires de géologie de Lyon 125: 187-202.

Guérin C., Curvello M. A., Faure M., Hugueney M. \& Mourer-Chauviré C. 1996a. - The Pleistocene fauna of Piauí (Northeastern Brazil). Palaeoecological and biochronological implications / A fauna pleistocênica do Piauí (Nordeste do Brasil). Relaçóes paleoecologicas e biocronologicas. FUMDHAMentos (Revista da Fundação do Homem Americano) 1 (1): 55-103 (bilingue anglais/portugais).

Guérin C., Galindo Lima M. \& PARENTI F. 1996b. La transition pléistocène/holocène à Conceição das Creoulas (Pernambouco, Brésil): mégafaune disparue et industries lithique. Actes du XIIIème Congrès de l'Union Internationale des Sciences Préhistoriques et Protohistoriques, Forli, septembre 1996, 5: 339-343.

Guidon N., Da Luz M. DE F., Guérin C. \& Faure M. 1993. — La Toca de Janela da Barra do Antonião et les autres sites paléolithiques karstiques de l'aire archéologique de São Raimundo Nonato (Piauí, Brésil) : état des recherches. Actes du XIIème Congrès de l'Union Internationale des Sciences Préhistoriques et Protohistoriques, Bratislava, septembre 1991, 3: 483-490.

Herbst R. \& Alvarez B. B. 1972. - Nota sobre los Toxodontes (Toxodontidae, Notungulata) del Cuartario de Corrientes, Argentina. Ameghiniana IX, 2: 149-158.
Hoffstetter R. 1986. - High Andean Mammalian faunas during the Plio-Pleistocene, in VuILLEUMIER F. \& Monasterio N. (éds), High Altitude Tropical Biogeography, Oxford University Press: 218-245.

Holland W. J. 1920. - Fossil Mammals collected at Pedra Vermelha, Bahia, Brazil, by Gerald A. Warring. Annals of Carnegie Museum XII: 224-232.

Kraglievich L. 1930-1931. — Bibliografia: Reivindicação do Genero Carolibergia, de Mercerat, por Alipio de Miranda-Ribeiro, Archivos do Museu Nacional, XXIX, paginas 9 a 20, laminas I a IV, Rio Janeiro 1927. Physis X, 35: 227-228.

Kraglievich L. 1931. — Sobre Trigodonops lopesi (Roxo) Kraglievich. Revista de la Sociedad Amigos de la Arqueologia, Montevideo, V: 195-200.

LAURITO C. A. 1993. - Analisis topologico y systematico del Toxodonte de Bajo de Los Barrantes, Provincia de Alajuela, Costa Rica. Revista Geológica de América Central 16: 61-68.

LaVocat R. 1958. - Notoungulata, in Piveteau J. (éd.), Traité de Paléontologie, 6, fasc. 2. Masson, Paris: 60-120.

LIAIS E. 1872. - Climats, géologie, faune et géographie botanique du Brésil. Garnier Frères, Paris, 640 p.

Lopes R. P., Oliveira L. C., Graciano Figueiredo A. M., Kinoshita A., Baffa O. \& SeKiguschi BuchMANN F. 2010. - ESR dating of pleistocene mammal teeth and its implications for the biostratigraphy and geological evolution of the coastal plain, Rio Grande do Sul, southern Brazil. Quaternary International 212: 213-222.

Lund P. W. 1950. - Memorias sobre a Paleontologia Brasileira, revistas e comentadas por Carlos de Paula Couto. Instituto nacional do Livro, Rio de Janeiro, $589 \mathrm{p}$.

MACFADDEN B. J. 2004. - Diet and habitat of Toxodont megaherbivores (Mammalia, Notoungulata) from the Late Quaternary of South and Central America. Journal of Vertebrate Paleontology 24 (3) suppl. : 86 A.

Marshall L. G., Berta A., Hoffstetter R., Pascual R., Reig O. A., Bombin M. \& Mones A. 1984. Mammals and stratigraphy: Geochronology of the continental mammal-bearing Quaternary of South America. Palaeovertebrata, Mém. extra.: 1-76.

McKenna M. C. \& Bell S. K. 1997. - Classification of Mammals Above the Species Level. Columbia University Press, New York, $631 \mathrm{p}$.

Melo D. J. De-, Henriques D. D. R. \& Carvalho C. R. 2007. - Ocorrência de defeitos de esmalte em materiais de Toxodon na coleção do museu nacional / UFRJ, uma perspecctiva paleoepidemiológica. Paleontologia: Cenários de Vida. Vol. 1. Interciência, Rio de Janeiro: 753-764.

MENDONÇA DOS SANTOS R. 2007. - Revisão dos toxodontes pleistcênicos brasileiros e consideraçôes sobre Trigodonops lopesi (Roxo, 1921) (Notoungulata, Toxodontidae). Dissertaçáo Mestrado, Instituto de Biociências, Departamento de Zoologia, Universidade de São Paulo 
(inédit): VII-X ; 1-18 ; 76-77 ; 98-104. http://www. museuhistorianatural.com/pesquisa_arquivos/2007/ Mendonca_2007_Dissertacao_Parcial.pdf (version partielle consultée par les auteurs).

MiÑo BoILINI A. R. \& BOND M. 2004. — Contribución al conocimiento del género Toxodon (Notoungulata: Toxodontidae) en el Pleistoceno de la provincia de Corrientes, Argentina. Comunicaciones Cientificas y Tecnológicas, Universidad Nacional del Nordeste, resumen B-027: 1-3.

Miño Boilini A. R., Cerdeño E. \& Bond M. 2006. Revisión del género Toxodon Owen, 1837 (Notoungulata: Toxodontidae) en el Pleistoceno de las provincias de Corrientes, Chaco y Santa Fe, Argentina. Revista Española de Paleontologia 21: 93-103.

Nasif N. L., Musalem S. \& Cerdeño E. 2000. — A new Toxodont from the Late Miocene of Catamarca, Argentina, and a phylogenetic analysis of the Toxodontidae. Journal of Vertebrate Paleontology 20 (3): 591-600.

Neves W., Hubbe A. \& Karmann I. 2007. - New accelerator mass spectrometry (AMS) ages suggest a revision of the electron spin resonance (ESR) middle Holocene dates obtained for a Toxodon platensis (Toxodontidae, Mammalia) from Southeast Brazil. Radiocarbon 49 (3): 1411-1412.

Oliveira É. V., Barreto A. M. F., \& Alves R. DA Silva. 2009. - Aspectos sistemáticos, paleobiogeográficos e paleoclimáticos dos mamíferos quaternários de Fazenda Nova, PE, nordeste do Brasil. Gaea-Journal of Geoscience 5 (2): 75-85.

Parenti F., Guidon N., Guérin C. \& Faure M. 1996. Les gisements pléistocènes de l'aire archéologique de São Raimundo Nonato (Piauí, Brésil). Actes du XIIIe Congrès international UISPP, Forli, septembre 1996, V: 345-349.

Parenti F., Guérin C., Mengoli D., Faure M., NAtali L., Miranda Chaves S.A. De-, Ferrari S. \& Manfra ValenÇA L. 2003. - Sondagens na Lagoa do Quari, São Raimundo Nonato, Piauí: campanha 2002. FUMDHAMentos 3: 129-145.

Pascual R. 1965. - Los Toxodontidae (Toxodonta, Notoungulata) de la Formacion Arroyo Chasico (Plioceno Inferior) de la Provincia de Buenos Aires. Caracteristicas geologicas. Ameghiniana IV, 4: 101-132.

Pascual R., Cattoi N. V., Francis J. C., Gondar D., Ortega Hinojosa E., Tonni E., Pisano J.A., RingueLET A. B. DE- \& ZETTI J. 1966. - IV- Vertebrata, in Borrelo A. V. (éd.), Paleontografía Bonaerense, Gobernación Provincia de Buenos Aires, Comisión de investigación científica, La Plata, 202 p.

Paula Couto C. DE 1942. - Um Toxodon no Pampeano do Rio Grande do Sul. Anais Academia brasileira de Ciências XIV (2): 137-142.

Paula Couto C. DE 1944. - Sobre a presença dos generos Hippidion e Toxodon Owen no Pleistoceno do Rio Grande do Sul. Boletim do Museu Nacional, Rio de Janeiro, NS, Geologia 2: 1-12.
Paula Couto C. DE 1954. — Sobre alguns mamíferos fósseis do Ceará. Arquivos Museu nacional Rio de Janeiro 42: 195-210.

Paula Couto C. De 1978. - Presença de Toxodon platensis Owen no Pleistoceno do Paraná. Iheringia, Série Geologia, 5 : 55-59.

Paula Couto C. DE 1979. - Tratado de Paleomastozoologia. Academia brasileira de Ciências, Rio de Janeiro, 590 p.

Paula Couto C. De 1980. - Fossil Pleistocene to Sub-Recent mammals from Northeastern Brazil. I Edentata Megalonychidae. Anais Academia brasileira de Ciências 52 (1): 143-151.

Paula Couto C. De 1981. - Fossil mammals from the Cenozoic of Acre, Brazil. IV-Notoungulata Notohippidae and Toxodontidae Nesodontinae. Anais II Congresso latino-americano de Paleontologia, Porto Alegre, avril 1981, vol. 2: 461-477.

Paula Couto C. De 1982. - Fossil mammals from the Cenozoic of Acre, Brazil. V - Notoungulata Nesodontinae (II), Toxodontinae and Haplodontiinae, and Litopterna, Pyrotheria and Astrapotheria (II). Iheringia, Série Geologia, Porto Alegre $7: 5$ - 43.

Perez Garcia M. I. 2000. — Morfotipos en la denticion del género Toxodon Owen, 1837 (Notoungulata, Toxodonta). Ameghiniana 37, n 4, Supl., Resúmenes: 31 R.

Peyre E., Guérin C., Guidon N. \& Coppens Y. 1998. Des restes humains pléistocènes dans la grotte de Garrincho, Piauí, Brésil. Comptes rendus de l'Académie des sciences de Paris 327: 335-360.

Porpino K. de Oliveira \& Santos M. de F. Cavalcante FerreIra dos 2003. - Novos registros de Artiodactyla e Perissodactyla para o Lagedo de Escada, Baraúna/ RN. XVIII Congresso brasileiro de Paleontologia, 13 a 18 de juhlo 2003, Boletim de resumos, Brasilia: 226.

Porpino K. de Oliveira, Santos M. de F. Cavalcante Ferreira dos \& BerQvist L. Paglarelli. 2004. Registros de Mamíferos fósseis no Lajedo de Soledade, Apodi, Rio Grande do Norte, Brasil. Revista Brasileira de Paleontologia 7 (3): 349-358.

PORTA J. DE 1959. - Nueva subespecie de toxodontido del Cuaternario de Colombia. Boletin de Geologia, Universidad industrial de Santander, Colombia 3: 55-61.

Prothero D. R., Guérin C. \& MANNing E. M. 1989. The history of the Rhinocerotoidea, in PROTHERO D. R. \& SCHOCH R. M. (éds), The evolution of Perissodactyls. IV Theriological Congress of Edmonton, Alberta, 1985, Oxford University Press, New-York: 321-340.

Ribeiro R. DA COSTA \& CARVALHO I. DE SOUZA 2009. Megafauna do Quaternário tardio de Baixa Grande, Bahia, Brasil. Anuário do Instituto de Geociências, UFRJ, 32, $2:$ 42-50.

Rolim J. L. 1971. - Sôbre alguns mamíferos fósseis de Lagoa da Pedra, municipio de Santa Cruz do Capibaribe, Pernambuco. Universidade federal de Pernambuco, Instituto de Geociências, sér. B., Estudos e pesquisas 1 (3): 1-19. 
Rolim J. L. 1974a. — Calcario secundario com restos fósseis de mamíferos pleistocênicos em Pernambuco. Anais Academia brasileira de Ciências 46, 3/4 : 417-422.

Rolim J. L. 1974b. - Paleontologia e estratigrafia do Pleistoceno continental do Nordeste brasileiro "Formação Cacimbas". Dissertaçáo de Mestrado, Universidade Federal do Rio Grande do Sul, Porto Alegre, 117 p. (inédit).

Roxo M. G. DE Oliveira 1921. — Note on a new species of Toxodon, Owen, T. Lopesi, Roxo. Empreza Brasil Editora, Rio de Janeiro: 1-7.

Saint-André P. A. 1999. — Les Toxodontidés du Néogène de l'Altiplano bolivien. Palaeontographica, Stuttgart A, $251: 79-148$.

Silva Alves R. Da \& Barreto A. M. F. 2007. - A megafauna pleistocênica de Fazenda Nova, Brejo da Madre de Deus, Pernambuco, Brasil. Paleontologia: Cenários de Vida vol. 1, Editora Interciência, Rio de Janeiro: 819-826.
Silva F. M., Cordeiro Filgueiras C. F., Barreto A. M. F. \& Oliveira É. V. 2010. - Mamíferos do Pleistoceno Superior de Afrânio, Pernambuco, nordeste do Brasil. Quaternary and Environmental Geosciences 2 (2): 1-11.

SHOCKEY B. J. 2001. - Specialized knee joints in some extinct, endemic South American herbivores. Acta Palaeontologica Polonica 46 (2): 277-288.

Souza Cunha F. L. DE 1959. - Mamíferos fósseis do Pleistoceno do Rio Grande do Sul. I - Ungulados. Departamento nacional da produção mineral, Divisão de Geologia e mineralogia, Rio de Janeiro, 202: 1-41.

VIDAL N. 1959. - Um Toxodon no Pleistoceno de Pernambuco. Boletim do Museu Nacional 30: 1-17.

WINGE H. 1906. — Jordfundne og nulevende Hovdyr (Ungulata) fra Lagoa Santa, Minas Geraes, Brasilien. Med Udsigt over Hovdyrenes indbyrdes Slaegtskab. E. Museo Lundii, Kjobenhavn, 3, 1: 1-239.

Soumis le 11 décembre 2010; accepté le 27 décembre 2011; publié le 29 mars 2013. 


\section{ANNEXES}

TABLEAU 1. - Dimensions comparées des crânes de Toxodon platensis Owen, 1837 et de Piauhytherium capivarae n. gen., n. sp. Abréviations : L, longueur; I, largeur; H, hauteur; DT, diamètre transversal; DAP, diamètre antéro-postérieur; prox, proximal; dia, diaphyse; dist, distal ; lat, latéral ; med, médial ; maxi, maximal ; artic, articulaire; SRN, São Raimundo Nonato; *, dimension approximative.

\begin{tabular}{|c|c|c|c|c|c|c|}
\hline \multirow[b]{2}{*}{ crâne } & \multirow[b]{2}{*}{$\mathrm{n}^{\circ}$ var. } & \multicolumn{4}{|c|}{ Piauhytherium capivarae n. gen., n. sp. } & \multirow{2}{*}{$\begin{array}{c}\begin{array}{c}\text { Toxodon } \\
\text { platensis }\end{array} \\
\text { Porcos } \\
\text { FUMDHAN } \\
186001\end{array}$} \\
\hline & & $\begin{array}{c}\text { Porcos } \\
\text { FUMDHAM } \\
188265\end{array}$ & $\begin{array}{c}\text { Porcos } \\
\text { FUMDHAM } \\
185391\end{array}$ & $\begin{array}{c}\text { São Vitor } \\
\text { FUMDHAM } \\
48947\end{array}$ & $\begin{array}{c}\text { Quari } \\
\text { FUMDHAM } \\
108998\end{array}$ & \\
\hline L condyles-prémaxillaires & 1 & 628 & 664 & & & 710 \\
\hline L occipito-nasale & 2 & 523 & & & & \\
\hline L nasal-POE & 3 & 539 & & & & \\
\hline L échancrure nasale & 4 & 86,5 & & & & \\
\hline I constriction post-o & 5 & 86 & 99 & 88 & 100 & 81,5 \\
\hline L POE-processus post-o & 6 & 235 & & & & \\
\hline L POE-processus sus-o & 7 & 265 & & & & \\
\hline L échancrure nasale-orbite & 8 & 157,5 & $140^{*}$ & & & 147 \\
\hline L ar. M3-condyle & 9 & 262 & & & & 326 \\
\hline L nasal-orbite & 10 & 242 & & & & \\
\hline I grande crête occipitale & 11 & 223 & & & & \\
\hline I aux apophyses mastoïdes & 12 & 356 & & 340 & 352 & $370^{\star}$ \\
\hline I mini. frontal & 13 & 148,5 & & & & 179 \\
\hline I processus post. orbitaire & 14 & 210 & & 193 & & 212 \\
\hline I bizygomatique & 15 & 403 & 364 & & & 423 \\
\hline I fond échancrure nasale & 16 & 93,5 & $98^{\star}$ & & & 103 \\
\hline H face occipale & 17 & 249 & & & & \\
\hline $\mathrm{H}$ niveau P2 & 18 & 215 & & & & \\
\hline H niveau P4-M1 & 19 & 243,5 & & & & \\
\hline $\mathrm{H}$ niveau M3 & 20 & $289^{*}$ & & & & \\
\hline I palais niveau P2 & 21 & 41,5 & 47 & & & 55,5 \\
\hline I palais niveau P4-M1 & 22 & 88 & 106 & & & \\
\hline I palais niveau M3 & 23 & 123 & 133,5 & & & $125^{\star}$ \\
\hline DT trou occipal & 24 & 58 & 67,5 & 65 & 70 & $72^{*}$ \\
\hline DT extér. condyles occip. & 25 & 164 & 151 & 162 & 155 & 195 \\
\hline | sur extérieur I2/ & 26 & 113 & 118 & & & 195 \\
\hline I constr. post-symphyse & 27 & 90 & 87 & & & 114 \\
\hline
\end{tabular}


TABLEAU 2. - Dimensions comparées des mandibules de Toxodon platensis Owen, 1837 et de Piauhytherium capivarae n. gen., n. sp. Abréviations : L, longueur ; I, largeur; H, hauteur; DT, diamètre transversal; DAP, diamètre antéro-postérieur; prox, proximal ; dia, diaphyse; dist, distal; lat, latéral; med, médial ; maxi, maximal; artic, articulaire; SRN, São Raimundo Nonato; *, dimension approximative.

\begin{tabular}{|c|c|c|c|c|}
\hline \multirow[b]{2}{*}{ mandibule } & \multicolumn{2}{|c|}{ P. capivarae n. gen., $\mathrm{n}$. sp. } & & \multirow{2}{*}{$\begin{array}{c}\text { T. platensis (Nordeste) } \\
\text { Porcos } \\
\text { FUMDHAM } \\
186001 \\
\end{array}$} \\
\hline & $\begin{array}{l}\text { São Vitor } \\
\text { FUMDHAM } \\
\text { SV 93-61 }\end{array}$ & $\begin{array}{c}\text { Porcos } \\
\text { FUMDHAM } \\
187141\end{array}$ & & \\
\hline Longueur & & 540 & & \\
\hline Dist. AR symphyse-talon & & 355 & & \\
\hline $\mathrm{H}$ br. horiz. $\mathrm{P} / 4-\mathrm{M} / 1$ & 113 & 118 & & 120 \\
\hline H br. horiz. M/2-/3 & 109,5 & 133 & & \\
\hline $\mathrm{H}$ br. horiz. après $\mathrm{M} / 3$ & & 157 & & \\
\hline DT br. horiz. sous P/4 & 45 & 49 & & 56 \\
\hline DT br. horiz. sous M/2-/3 & 43 & 54 & & \\
\hline \multirow{7}{*}{$\begin{array}{l}\text { L symphyse } \\
\text { H maxi symphyse } \\
\text { DAP br. montante niv. table } \\
\text { DT condyle } \\
\text { H condyle }\end{array}$} & & 231 & & 209 \\
\hline & & 87 & & \\
\hline & & 194 & & \\
\hline & & 99,5 & & $100^{*}$ \\
\hline & & 356 & & \\
\hline & \multicolumn{4}{|c|}{ T. platensis (Argentine) } \\
\hline & nombre & moyenne & $\operatorname{mini}$ & $\operatorname{maxi}$ \\
\hline Dist. AR symphyse-talon & 2 & 328,5 & 305 & 352 \\
\hline H br. horiz. P/4- M/1 & 3 & 140,83 & 123 & 150 \\
\hline H br. horiz. M/2-/3 & 2 & 151,75 & 140 & 163,5 \\
\hline $\mathrm{H}$ br. horiz. après $\mathrm{M} / 3$ & 1 & 61 & & \\
\hline DT br. horiz. sous P/4 & 2 & 61,25 & 53,5 & 69 \\
\hline \multirow{5}{*}{\multicolumn{5}{|c|}{$\begin{array}{l}\text { DT br. horiz. sous } \mathrm{M} / 2-/ 3 \\
\text { L symphyse } \\
\text { H maxi symphyse } \\
\text { DAP br. montante niv. table }\end{array}$}} \\
\hline & & & & \\
\hline & & & & \\
\hline & & & & \\
\hline & & & & \\
\hline H condyle & 2 & 362,5 & 340 & 385 \\
\hline
\end{tabular}


TABleau 3. - Dimensions comparées des dents jugales supérieures des Toxodontidae (Toxodon platensis Owen, 1837 et Piauhytherium capivarae n. gen., n. sp.) de la région du Parc national Serra da Capivara (SE du Piauí, Brésil). Abréviations : L, longueur; I, largeur; H, hauteur; D, droit ; G, gauche ; DT, diamètre transversal; DAP, diamètre antéro-postérieur; prox, proximal; dia, diaphyse; dist, distal; lat, latéral; med, médial; maxi, maximal; artic, articulaire; SRN, São Raimundo Nonato; *, dimension approximative.

\section{Piauhytherium capivarae n. gen., n. sp.}

\begin{tabular}{|c|c|c|c|c|c|c|c|c|c|c|c|c|c|c|}
\hline dents sup. & 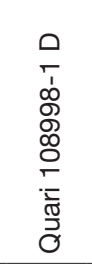 & 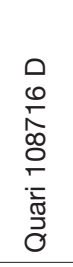 & 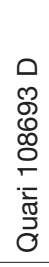 & 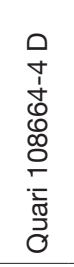 & 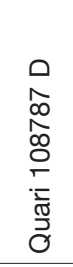 & 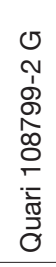 & 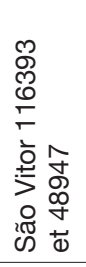 & 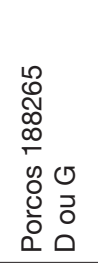 & 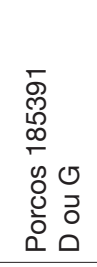 & $\begin{array}{l}0 \\
0 \\
0 \\
0 \\
0 \\
\infty \\
0 \\
0 \\
0 \\
0 \\
0 \\
0\end{array}$ & 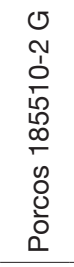 & 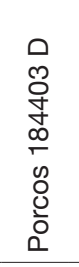 & 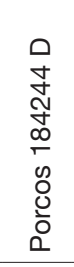 & $\begin{array}{l}0 \\
0 \\
م \\
\infty \\
\infty \\
0 \\
0 \\
0 \\
0 \\
0 \\
0\end{array}$ \\
\hline P 1/L & & & & & & 14 & & 15,5 & 18,5 & & & & & \\
\hline P 1/I & & & & & & 17 & & 23,5 & 18,5 & & & & & \\
\hline P 2/ L ectol. & & & & & & & & 23 & 22 & & & & & \\
\hline $\mathrm{P} 2 / \mathrm{L}$ anat & & & & & & & & & & & & & & \\
\hline P 2/I anat. & & & & & & & & & & & & & & \\
\hline P 2/I maxi & & & & & & & & 24,5 & 26,5 & & & & & \\
\hline P 3/ L ectol. & & & 39 & 38 & & & & 28,5 & 29 & & & & & \\
\hline P 3/ L anat & & & 25 & 25,5 & & & & 28 & & & & & & \\
\hline P 3/ I anat & & & 25 & 26 & & & & & & & & & & \\
\hline P 3/ I maxi & & & 33 & 29 & & & & 30 & 32 & & & & & \\
\hline P 4/ L ectol. & 49,5 & 40 & & & 48 & & 37 & 37 & 38,5 & & & & & \\
\hline P 4/ L. anat & 32 & 32 & & & 31 & & 32,5 & 38 & 44 & & & & & \\
\hline P 4/I. anat & 28 & 28 & & & 29,5 & & 31 & 35 & & & & & & \\
\hline P 4/I. maxi & 39 & 37,5 & & & 43,5 & & 40,5 & 35 & 32 & & & & & \\
\hline M 1/ L ectol. & 67,5 & & & & & & 66 & 63 & 64 & 66 & & & & 65 \\
\hline M 1/ L anat. & 38,5 & & & & & & 40 & 56,5 & 52 & 50 & & & & 48,5 \\
\hline M 1/I anat & 32 & & & & & & 34 & 37,5 & 32 & 30,5 & & & & 32,5 \\
\hline M 1/I maxi & 45,5 & & & & & & 48 & 40 & & & & & & \\
\hline M 2/ L ectol. & 67,5 & & & & & & & 62,5 & 65,5 & & 68 & 69 & 70,5 & \\
\hline M 2/ L anat. & 43 & & & & & & & 62,5 & 50 & & 51 & 50,5 & 52,5 & \\
\hline M 2/ I anat. & 34 & & & & & & & 40 & 49 & & 31,5 & 30,5 & 31,5 & \\
\hline M 2/ I maxi. & 51,5 & & & & & & & 54 & & & & & & \\
\hline M 3/ L ectol. & 67,5 & & & & & & 71 & 65 & 64 & & & & & \\
\hline M 3/ L anat. & 45,5 & & & & & & 44 & 68,5 & 59 & & & & & \\
\hline M 3/ I. anat. & 33 & & & & & & 31 & 35 & 42,5 & & & & & \\
\hline M 3/ I maxi. & 48 & & & & & & 52 & 35 & & & & & & \\
\hline L P1/-M3/ & & & & & & & & & 257,5 & & & & & \\
\hline L P2/-M3/ & & & & & & & & 257 & & & & & & \\
\hline$L$ des $P$ & & & & & & & & 114 & 118 & & & & & \\
\hline L P3/-P4/ & & & & & & & & 66,5 & 67,5 & & & & & \\
\hline$L$ des $M$ & 160 & & & & & & & 158 & 150 & & & & & \\
\hline
\end{tabular}


TABleau 3. - Suite.

Piauhytherium capivarae n. gen., n. sp.

\begin{tabular}{|c|c|c|c|c|c|c|c|c|c|c|c|c|c|}
\hline dents sup. & 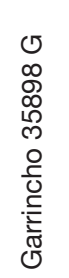 & 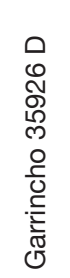 & 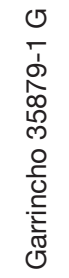 & 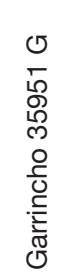 & 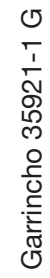 & 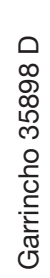 & 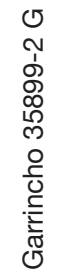 & 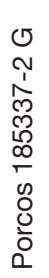 & 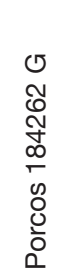 & $\begin{array}{l}0 \\
\text { m } \\
\infty \\
\bar{N} \\
\infty \\
- \\
\infty \\
0 \\
0 \\
0 \\
0\end{array}$ & 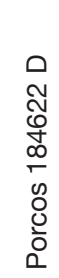 & \begin{tabular}{l}
0 \\
$\infty$ \\
\multicolumn{1}{c}{} \\
$\bar{N}$ \\
$\infty$ \\
$\infty$ \\
0 \\
0 \\
0 \\
0 \\
0
\end{tabular} & 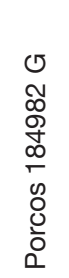 \\
\hline \multicolumn{14}{|l|}{$\mathrm{P} 1 / \mathrm{L}$} \\
\hline P 2/ L ectol. & & & & & 29 & 30 & & & 25 & & & 24 & \\
\hline P 2/ L anat & & & & & 26 & 25 & & & & & & & \\
\hline P 2/ I anat. & & & & & 24 & 24 & & & 31 & & & 26 & \\
\hline P 2/ I maxi & & & & & 23 & 25 & & & & & & & \\
\hline P 3/ L ectol. & & & & 44 & & & & 28 & & 30 & 31 & & 34 \\
\hline P 3/ L anat & & & & 32 & & & & 25 & & & & & 22,5 \\
\hline P 3/ I anat & & & & 28,5 & & & & & & 26,5 & 30 & & \\
\hline P 3/ I maxi & & & & 34 & & & & & & & & & \\
\hline P 4/ L ectol. & & & 51,5 & & & & & & & & & & \\
\hline P 4/ L. anat & & & 30 & & & & & & & & & & \\
\hline P 4/ I. anat & & & 28,5 & & & & & & & & & & \\
\hline P 4/ I. maxi & & & 49 & & & & & & & & & & \\
\hline \multicolumn{14}{|l|}{ M 1/ L ectol. } \\
\hline \multicolumn{14}{|l|}{ M 1/ L anat. } \\
\hline \multicolumn{14}{|l|}{ M 1/ I anat } \\
\hline \multicolumn{14}{|l|}{ M 1/ I maxi } \\
\hline M 2/ L ectol. & 66 & & & & & & & & & & & & \\
\hline M 2/ L anat. & 39,5 & & & & & & & & & & & & \\
\hline M 2/ I anat. & 32 & & & & & & & & & & & & \\
\hline M 2/ I maxi. & 58 & & & & & & & & & & & & \\
\hline M 3/ L ectol. & & 77 & & & & & 71,5 & & & & & & \\
\hline M 3/ L anat. & & 46 & & & & & 42,5 & & & & & & \\
\hline M 3/ I. anat. & & 31,5 & & & & & 29,5 & & & & & & \\
\hline M 3/ I maxi. & & 65 & & & & & 63,5 & & & & & & \\
\hline L P2/-M3/ & & & & & & & & & & & & & \\
\hline$L$ des $P$ & & & & & & & & & & & & & \\
\hline L P3/-P4/ & & & & & & & & & & & & & \\
\hline L des M & & & & & & & & & & & & & \\
\hline
\end{tabular}


TAbleau 3. - Suite.

\begin{tabular}{|c|c|c|c|c|c|c|c|c|c|c|c|}
\hline \multirow[b]{2}{*}{$\begin{array}{l}\text { Jugales } \\
\text { supérieures }\end{array}$} & \multicolumn{7}{|c|}{ Toxodon platensis } & \multicolumn{4}{|c|}{ Toxodon platensis (Argentine) } \\
\hline & 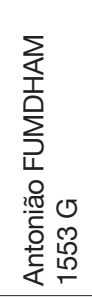 & 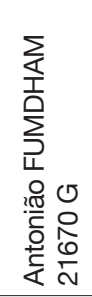 & 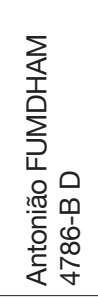 & 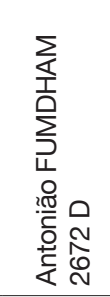 & 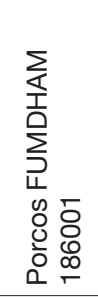 & 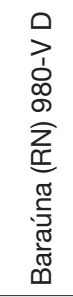 & 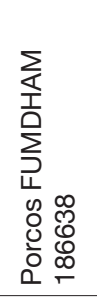 & $\begin{array}{l}\text { @) } \\
\text { है } \\
\text { हे }\end{array}$ & 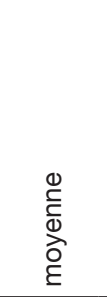 & 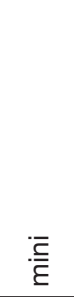 & $\begin{array}{l}\bar{x} \\
\stackrel{\mathbb{\varpi}}{\varepsilon}\end{array}$ \\
\hline $\mathrm{P} 1 / \mathrm{L}$ & & & & & & & & 1 & 6,50 & & \\
\hline P $1 / I$ & & & & & & & & 1 & 5,50 & & \\
\hline P 2/ L ectol. & 29 & & & & 21 & & & 1 & 20,00 & & \\
\hline $\mathrm{P} 2 / \mathrm{L}$ anat. & 20,5 & & & & 20 & & & 0 & & & \\
\hline P 2/ I anat. & 18 & & & & & & & 1 & 16,00 & 16 & 16 \\
\hline P 2/I maxi & 25 & & & & & & & 0 & & & \\
\hline P 3/ L ectol. & & & 24,5 & 25 & & & & 3 & 36,50 & 33,5 & 38,5 \\
\hline P 3/ L anat. & & & 25,5 & 24 & & & & 1 & 22,50 & & \\
\hline P 3/ I anat. & & & 27 & 28 & & & & 3 & 20,50 & 16,5 & 22,5 \\
\hline P 3/ I maxi & & & 22,5 & 22,5 & & & & 1 & 24,00 & & \\
\hline P 4/ L ectol. & & 36 & & & 42 & & & 4 & 47,13 & 45 & 48,5 \\
\hline P 4/ L. anat. & & 26,5 & & & 30 & & & 1 & 26,50 & & \\
\hline P 4/ I. anat. & & & & & & & & 4 & 24,38 & 22 & 27 \\
\hline P 4/I. maxi & & 27 & & & & & & 1 & 27,50 & & \\
\hline M 1/ L ectol. & & & & & & & & 3 & 63,67 & 56,5 & 72,5 \\
\hline M 1/ L anat. & & & & & & & & 2 & 42,25 & 42 & 42,5 \\
\hline M 1/ I anat. & & & & & & & & 3 & 32,33 & 27 & 37 \\
\hline M 1/ I maxi & & & & & & & & 2 & 46,75 & 40,5 & 53 \\
\hline M 2/ L ectol. & & & & & & & 67,5 & 3 & 67,67 & 60,5 & 73,5 \\
\hline M 2/ L anat. & & & & & & & 46 & 2 & 47,00 & 38 & 56 \\
\hline M 2/ I anat. & & & & & & & 28 & 4 & 30,13 & 28 & 33 \\
\hline M 2/I maxi & & & & & & & & 3 & 47,33 & 42 & 53,5 \\
\hline M 3/ L ectol. & & & & & & 65 & & 3 & 64,50 & 60,5 & 68 \\
\hline M 3/ L anat. & & & & & & 45 & & 2 & 51,50 & 45 & 58 \\
\hline M 3/ I. anat. & & & & & & 27,5 & & 3 & 29,00 & 27,5 & 31 \\
\hline M 3/I maxi & & & & & & & & 1 & 40,00 & & \\
\hline L P1/-M3/ & & & & & 254 & & & & & & \\
\hline L P2/-M3/ & & & & & 229 & & & 1 & 207,00 & & \\
\hline$L \operatorname{des} P$ & & & & & & & & 1 & 81,00 & & \\
\hline L P3/-P4/ & & & & & 65 & & & 1 & 52,50 & & \\
\hline$L$ des $M$ & & & & & 152 & & & 1 & 138,00 & & \\
\hline
\end{tabular}


TABLEAU 4. - Dimensions comparées des dents jugales inférieures des Toxodontidae (Toxodon platensis Owen, 1837 et Piauhytherium capivarae n. gen., n. sp.) de la région du Parc national Serra da Capivara (SE du Piauí, Brésil). Abréviations : L, longueur; I, largeur; H, hauteur; D, droit ; G, gauche ; DT, diamètre transversal; DAP, diamètre antéro-postérieur; prox, proximal; dia, diaphyse; dist, distal; lat, latéral; med, médial; maxi, maximal; artic, articulaire; SRN, São Raimundo Nonato; *, dimension approximative.

Piauhytherium capivarae n. gen., n. sp.

\begin{tabular}{|c|c|c|c|c|c|c|c|c|c|c|c|c|c|c|c|}
\hline $\begin{array}{l}\text { Jugales } \\
\text { inférieures }\end{array}$ & 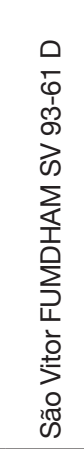 & 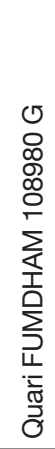 & 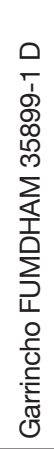 & 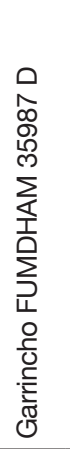 & 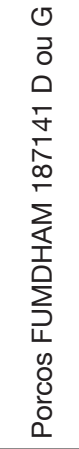 & 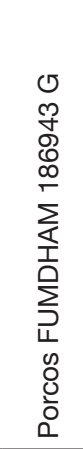 & 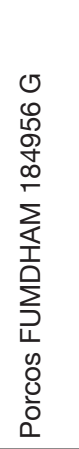 & 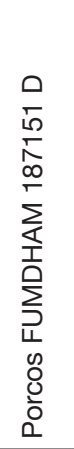 & 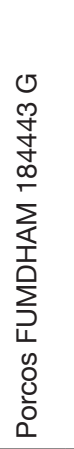 & 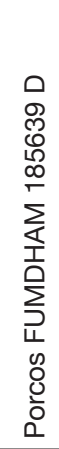 & 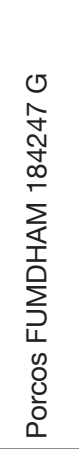 & 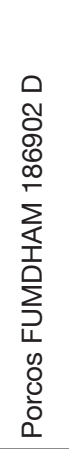 & 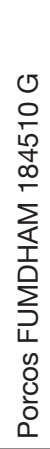 & 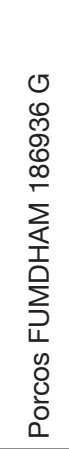 & 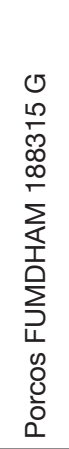 \\
\hline & & & & & & & \multicolumn{4}{|c|}{$P / 1 L$} & & & & & \\
\hline P /2 L & & & & & 23,5 & & & & & & & & 18 & & \\
\hline P /2 I ant. & & & & & 14,5 & & & & & & & & & & \\
\hline P /2 I post. & & & & & 16 & & & & & & & & 17 & & \\
\hline $\mathrm{P} / 3 \mathrm{~L}$ & & & & 31,5 & 30 & 28,5 & & & & & & & & & 29 \\
\hline P/3 I ant. & & & & 13 & 15 & 13,5 & & & & & & & & & 12,5 \\
\hline P /3 I post. & & & & 18 & 18,5 & 16 & & & & & & & & & 16 \\
\hline P /4 L & 29,5 & & & & 34 & 34,5 & & & & & & & & 32 & \\
\hline P /4 I ant. & 18 & & & & 18 & 16,5 & & & & & & & & 16,5 & \\
\hline P /4 I post. & 19 & & & & 20 & 18 & & & & & & & & 19,5 & \\
\hline $\mathrm{M} / 1 \mathrm{~L}$ & 46,5 & 48 & & & 54 & 48 & & & 43,5 & 47 & 43,5 & & & & \\
\hline M /1 I ant. & 17,5 & 19 & & & 22,5 & 19,5 & & & 17 & 18 & 19 & & & & \\
\hline M /1 I post. & 16,5 & 18 & & & 19,5 & 17 & & & 15,5 & 16 & 15 & & & & \\
\hline $\mathrm{M} / 2 \mathrm{~L}$ & 44 & & 50 & & 55,5 & 47 & & & & & & 50 & & & \\
\hline M /2 I ant. & 19 & & 20 & & 25 & 22 & & & & & & 20 & & & \\
\hline M /2 I post. & 14,5 & & 15 & & 18,5 & 16 & & & & & & 18,5 & & & \\
\hline $\mathrm{M} / 3 \mathrm{~L}$ & 58 & & & & 65,5 & 59,5 & 66 & 61,5 & & & & & & & \\
\hline M /3 I. ant. & 20 & & & & 21,5 & 20 & 20,5 & 19,5 & & & & & & & \\
\hline M /3 I post. & 12,5 & & & & 14,5 & 15 & 13 & 14 & & & & & & & \\
\hline L P/1-M/3 & & & & & 277 & & & & & & & & & & \\
\hline L P/2-M/3 & & & & & 259 & & & & & & & & & & \\
\hline L des P & & & & & 112 & & & & & & & & & & \\
\hline L 2 dern. /P & & & & & 73,5 & 61 & & & & & & & & & \\
\hline L des /M & 148 & & & & 169 & 161 & & & & & & & & & \\
\hline
\end{tabular}


TABleau 4. - Suite.

\begin{tabular}{|c|c|c|c|c|c|c|c|c|c|c|c|c|}
\hline \multirow[b]{2}{*}{$\begin{array}{l}\text { Jugales } \\
\text { inférieures }\end{array}$} & \multicolumn{6}{|c|}{ T. platensis (Nordeste) } & \multicolumn{6}{|c|}{ T. platensis (Argentine) } \\
\hline & 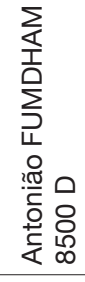 & 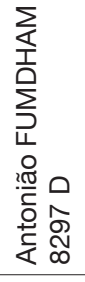 & 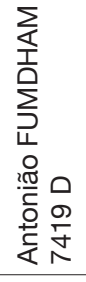 & 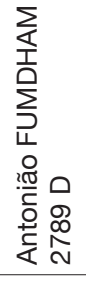 & 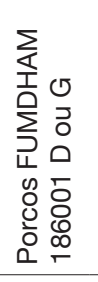 & 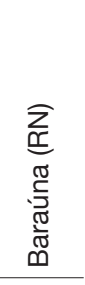 & 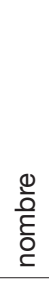 & 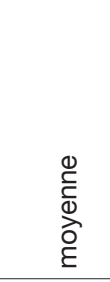 & $\overline{\bar{E}}$ & $\begin{array}{l}\bar{x} \\
\stackrel{\mathbb{\varpi}}{\varepsilon} \\
\end{array}$ & 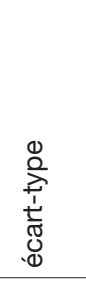 & 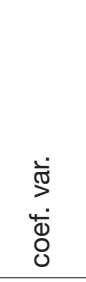 \\
\hline $\mathrm{P} / 1 \mathrm{~L}$ & & & & & & & 1 & 11,50 & & & & \\
\hline $\mathrm{P} / 1 \mathrm{I}$ & & & & & & & 1 & 9,50 & & & & \\
\hline $\mathrm{P} / 2 \mathrm{~L}$ & & & & & & & 3 & 23,33 & 21 & 26 & & \\
\hline P /2 I ant. & & & & & & & 2 & 9,75 & 9 & 10,5 & & \\
\hline P /2 I post. & & & & & & & 3 & 12,00 & 9,5 & 13,5 & & \\
\hline $\mathrm{P} / 3 \mathrm{~L}$ & 26 & & & & 27 & & 3 & 24,33 & 21,5 & 27 & & \\
\hline P /3 I ant. & & & & & 11,5 & & 3 & 10,50 & 9 & 12,5 & & \\
\hline $\mathrm{P} / 3$ I post. & 13 & & & & 15,5 & & 3 & 13,83 & 11 & 17,5 & & \\
\hline $\mathrm{P} / 4 \mathrm{~L}$ & & & & & 30 & & 4 & 29,13 & 26,5 & 33,5 & & \\
\hline P /4 I ant. & & & & & & & 4 & 14,38 & 13 & 17,5 & & \\
\hline P /4 I post. & & & & & 19 & & 4 & 15,75 & 14 & 20 & & \\
\hline $\mathrm{M} / 1 \mathrm{~L}$ & & & & & 52 & & 3 & 44,17 & 39,5 & 48 & & \\
\hline M /1 I ant. & & & & & 17,5 & & 3 & 17,33 & 15 & 20,5 & & \\
\hline M /1 I post. & & & & & & & 2 & 17,25 & 15 & 19,5 & & \\
\hline $\mathrm{M} / 2 \mathrm{~L}$ & & 40 & & & & 43,5 & 8 & 43,13 & 40 & 50 & 3,15 & 7,30 \\
\hline M /2 I ant. & & 17 & & & & 18,5 & 8 & 18,69 & 15 & 21 & 1,96 & 10,50 \\
\hline M /2 I post. & & 15 & & & & 15,5 & 7 & 16,21 & 14,5 & 18,5 & 1,35 & 8,32 \\
\hline$M / 3 L$ & & & 58 & 59 & 65 & & 7 & 57,64 & 51,5 & 66,5 & 4,81 & 8,35 \\
\hline M /3 I. ant. & & & 14,5 & 17 & 18,5 & & 7 & 16,71 & 13 & 19,5 & 2,38 & 14,23 \\
\hline M /3 I post. & & & 11 & 11 & & & 6 & 11,42 & 10 & 12,5 & 0,92 & 8,04 \\
\hline L RD & & & & & 247,5 & & 2 & 217,75 & 213,5 & 222 & & \\
\hline$L$ des $P$ & & & & & & & 2 & 76,75 & 74,5 & 79 & & \\
\hline L 2 dern. /P & & & & & 54,5 & & 4 & 56,75 & 51,5 & 64 & 6,28 & \\
\hline$L$ des $/ M$ & & & & & 154 & & 5 & 151,30 & 142,5 & 165 & 9,14 & 6,04 \\
\hline
\end{tabular}


TABleAu 5. - Dimensions comparées des humérus des Toxodontidae (Toxodon platensis Owen, 1837 et Piauhytherium capivarae n. gen., n. sp.) de la région du Parc national Serra da Capivara (SE du Piauí, Brésil) et d'autres sites pléistocènes du Brésil. Abréviations : L, longueur; I, largeur; H, hauteur; D, droit ; G, gauche ; DT, diamètre transversal; DAP, diamètre antéro-postérieur; prox, proximal; dia, diaphyse; dist, distal; lat, latéral; med, médial; maxi, maximal; artic, articulaire; SRN, São Raimundo Nonato; *, dimension approximative.

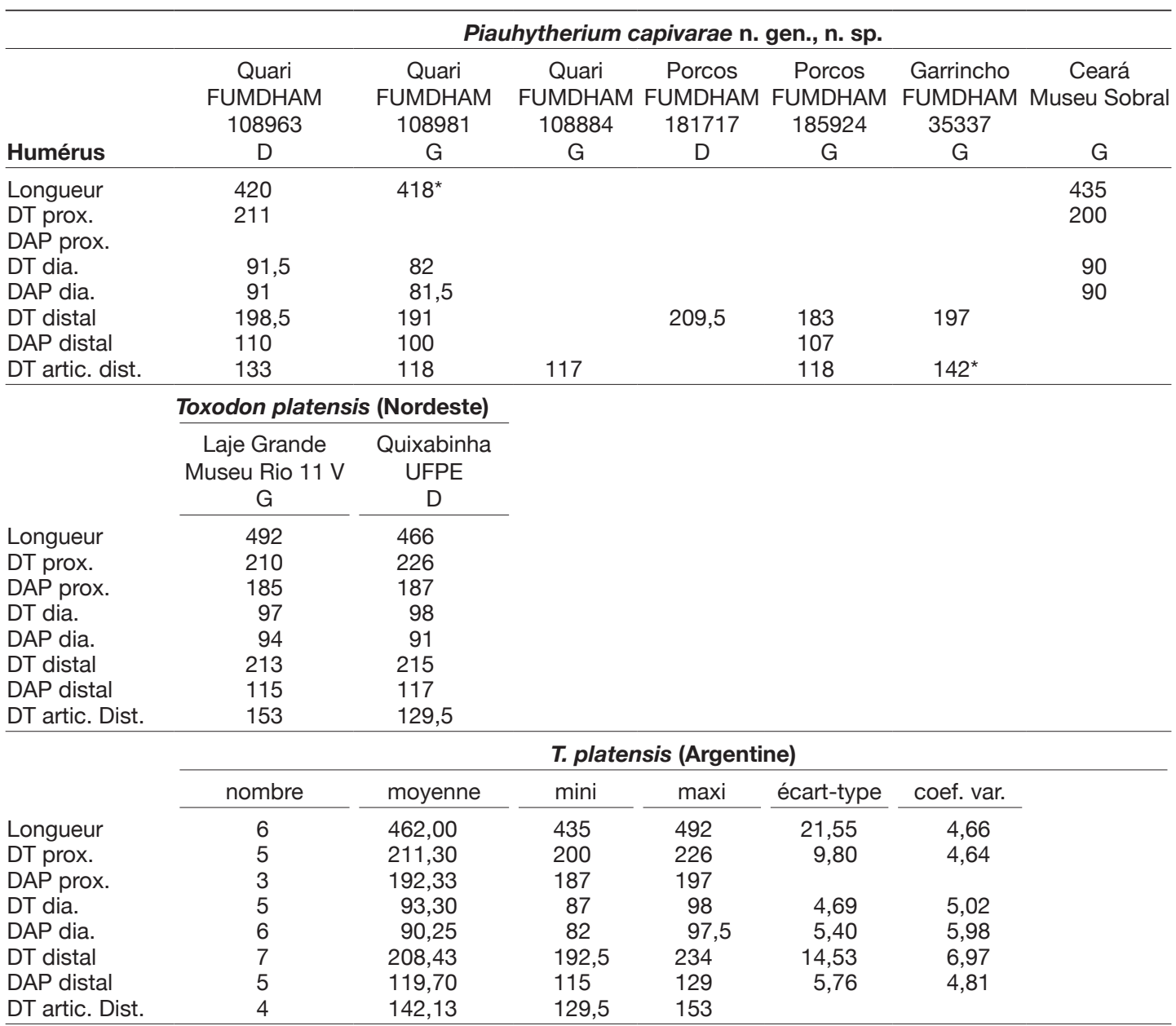


Tableau 6. - Dimensions comparées des radius des Toxodontidae (Toxodon platensis Owen, 1837 et Piauhytherium capivarae n. gen., n. sp.) de la région du Parc national Serra da Capivara (SE du Piauí, Brésil) et d'autres sites pléistocènes du Brésil. Abréviations : L, longueur; I, largeur; H, hauteur; D, droit ; G, gauche ;DT, diamètre transversal ; DAP, diamètre antéro-postérieur; prox, proximal ; dia, diaphyse; dist, distal; lat, latéral ; med, médial; maxi, maximal; artic, articulaire; SRN, São Raimundo Nonato; * , dimension approximative.

\begin{tabular}{|c|c|c|c|c|c|c|}
\hline \multirow[b]{2}{*}{ Radius } & \multicolumn{2}{|c|}{ P. capivarae n. gen., n. sp. } & & & \multicolumn{2}{|c|}{ T. platensis (Nordeste) } \\
\hline & \multicolumn{2}{|c|}{$\begin{array}{c}\text { Porcos } \\
\text { FUMDHAM } \\
184373 \text { D }\end{array}$} & & & $\begin{array}{c}\text { Garrincho (PI) } \\
\text { FUMDHAM } \\
\text { 35978-1 G }\end{array}$ & $\begin{array}{c}\text { Laje Grande (PE) } \\
\text { Museu Rio } \\
15 \text { V G }\end{array}$ \\
\hline L radius & \multicolumn{2}{|c|}{325} & & & & 340 \\
\hline DT prox. maxi & \multicolumn{2}{|c|}{73,5} & & & & 72 \\
\hline DAP prox. anat. & \multicolumn{2}{|c|}{47} & & & & 62 \\
\hline DT dia. & \multicolumn{2}{|c|}{39,5} & & & & 47 \\
\hline DAP dia. & \multicolumn{2}{|c|}{41} & & & & 49 \\
\hline DT dist. & \multicolumn{2}{|c|}{87} & & & 101,5 & 106 \\
\hline \multirow[t]{3}{*}{ DAP dist. } & \multicolumn{2}{|c|}{85} & & & $76^{\star}$ & 89 \\
\hline & \multicolumn{6}{|c|}{ T. platensis (Argentine) } \\
\hline & nombre & moyenne & $\operatorname{mini}$ & $\operatorname{maxi}$ & écart-type & coef. var. \\
\hline$L$ radius & 4 & 341,88 & 336 & 357 & 10,12 & 2,96 \\
\hline DT prox maxi & 3 & 79,17 & 76 & 82,5 & 3,25 & \\
\hline DAP prox anat & 3 & 55,17 & 54 & 56,5 & 1,26 & \\
\hline DT dia & 3 & 48,33 & 46,5 & 49,5 & 1,61 & \\
\hline DAP dia & 3 & 50,17 & 43,5 & 55 & 5,97 & \\
\hline DT dist. & 3 & 118,00 & 106 & 128 & 11,14 & \\
\hline DAP dist. & 3 & 88,00 & 81 & 98 & 8,89 & \\
\hline
\end{tabular}

TABlEaU 7. - Dimensions comparées des Métacarpiens II des Toxodontidae (Toxodon platensis Owen, 1837 et Piauhytherium capivarae n. gen., n. sp.) de la région du Parc national Serra da Capivara (SE du Piauí, Brésil) et d'autres sites pléistocènes du Brésil. Abréviations : L, longueur; I, largeur; H, hauteur; D, droit ; G, gauche ; DT, diamètre transversal; DAP, diamètre antéro-postérieur; prox, proximal; dia, diaphyse; dist, distal; lat, latéral; med, médial; maxi, maximal; artic, articulaire; SRN, São Raimundo Nonato; *, dimension approximative.

\begin{tabular}{|c|c|}
\hline \multirow[b]{2}{*}{ Mc II } & P. capivarae n. gen., n. sp. \\
\hline & Lagoa Pedra UFPE LP 43-5 \\
\hline Longueur & 116 \\
\hline DT prox & \\
\hline DAP prox & \\
\hline DT dia. & 37 \\
\hline DAP dia. & 27,5 \\
\hline DT distal & 52 \\
\hline DT artic. distal & 43,5 \\
\hline DAP artic. dist. & 43,5 \\
\hline
\end{tabular}

\begin{tabular}{|c|c|c|c|c|c|c|}
\hline & & & ensis (A & tine) & & \\
\hline & nombre & moyenne & $\operatorname{mini}$ & $\operatorname{maxi}$ & écart-type & coef. var. \\
\hline Longueur & 5 & 147,60 & 137,5 & 159,5 & 8,90 & 6,03 \\
\hline DT prox & 4 & 62,38 & 60 & 65 & 2,06 & 3,30 \\
\hline DAP prox & 4 & 63,50 & 61 & 65 & 1,91 & 3,02 \\
\hline DT dia. & 5 & 43,00 & 39 & 49 & 4,14 & 9,62 \\
\hline DAP dia. & 5 & 34,00 & 32 & 36 & 1,58 & 4,65 \\
\hline DT distal & 5 & 63,00 & 60,5 & 67 & 2,72 & 4,31 \\
\hline DT artic. distal & 5 & 55,30 & 51 & 61 & 3,83 & 6,93 \\
\hline DAP artic. dist. & 4 & 53,25 & 50 & 59 & 4,13 & 7,76 \\
\hline
\end{tabular}


TABleau 7. - Suite.

\section{T. platensis (Nordeste)}

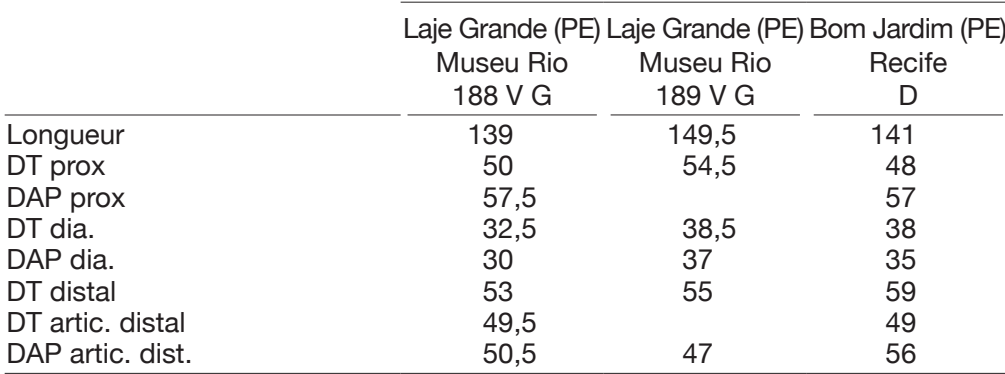

TABleau 8. - Dimensions comparées des Métacarpiens III des Toxodontidae (Toxodon platensis Owen, 1837 et Piauhytherium capivarae n. gen., n. sp.) de la région du Parc national Serra da Capivara (SE du Piauí, Brésil) et d'autres sites pléistocènes du Brésil. Abréviations : L, longueur; I, largeur; H, hauteur; D, droit ; G, gauche ; DT, diamètre transversal; DAP, diamètre antéro-postérieur; prox, proximal ; dia, diaphyse; dist, distal ; lat, latéral ; med, médial ; maxi, maximal ; artic, articulaire; SRN, São Raimundo Nonato; *, dimension approximative.

\begin{tabular}{|c|c|c|c|c|c|c|c|}
\hline \multirow[b]{2}{*}{ Mc III } & \multicolumn{4}{|c|}{ Piauhytherium capivarae n. gen., n. sp. } & \multicolumn{3}{|c|}{ Toxodon platensis (Brésil) } \\
\hline & $\begin{array}{c}\text { Quari } \\
\text { FUMDHAM } \\
109005 \\
\text { G }\end{array}$ & $\begin{array}{l}\text { São Vitor } \\
\text { FUMDHAM } \\
48909\end{array}$ & & $\begin{array}{l}\text { rcos } \\
\text { DHAM } \\
5530 \\
\text { G }\end{array}$ & & $\begin{array}{c}\text { V do Palmar } \\
\text { Museu Rio } \\
2.619 \mathrm{U} \\
\text { D }\end{array}$ & $\begin{array}{c}\text { Lagoa Santa } \\
\text { Copenhague } \\
3355\end{array}$ \\
\hline $\begin{array}{l}\text { Longueur } \\
\text { DT prox. } \\
\text { DAP prox. } \\
\text { DT dia. } \\
\text { DAP dia. } \\
\text { DT distal } \\
\text { DT artic. distal } \\
\text { DAP artic. dist. }\end{array}$ & $\begin{array}{l}131 \\
55,5 \\
54,5 \\
46,5 \\
27 \\
67,5 \\
56 \\
47,5 \\
\end{array}$ & $\begin{array}{l}>115 \\
>51 \\
>42 \\
52 \\
26,5 \\
60,5 \\
52 \\
40 \\
\end{array}$ & & $\begin{array}{l}58,5 \\
28,5 \\
74 \\
71,5 \\
52,5\end{array}$ & & $\begin{array}{l}164 \\
72 \\
68 \\
65 \\
32 \\
84,5 \\
65 \\
50 \\
\end{array}$ & 75 \\
\hline \multirow{2}{*}{ DAP artic. dist. } & \multicolumn{6}{|c|}{ T. platensis (Argentine) } & \\
\hline & nombre & moyenne & $\operatorname{mini}$ & $\operatorname{maxi}$ & écart-type & coef. var. & \\
\hline $\begin{array}{l}\text { Longueur } \\
\text { DT prox. } \\
\text { DAP prox. } \\
\text { DT dia. } \\
\text { DAP dia. } \\
\text { DT distal } \\
\text { DT artic. distal } \\
\text { DAP artic. dist. }\end{array}$ & $\begin{array}{l}6 \\
6 \\
5 \\
5 \\
5 \\
5 \\
5 \\
4\end{array}$ & $\begin{array}{r}156,92 \\
77,42 \\
71,80 \\
63,20 \\
33,90 \\
80,20 \\
65,60 \\
54,13\end{array}$ & $\begin{array}{l}145 \\
72 \\
68 \\
58,5 \\
27,5 \\
73,5 \\
61,5 \\
50\end{array}$ & $\begin{array}{l}164 \\
83 \\
78 \\
66,5 \\
38,5 \\
86 \\
71 \\
57\end{array}$ & $\begin{array}{l}6,87 \\
4,39 \\
4,27 \\
3,49 \\
4,55 \\
5,08 \\
3,52 \\
3,12\end{array}$ & $\begin{array}{r}4,38 \\
5,67 \\
5,94 \\
5,53 \\
13,41 \\
6,34 \\
5,37 \\
5,76\end{array}$ & \\
\hline
\end{tabular}


TABlEAU 9. - Dimensions comparées des Métacarpiens IV des Toxodontidae (Toxodon platensis Owen, 1837 et Piauhytherium capivarae n. gen., n. sp.) de la région du Parc national Serra da Capivara (SE du Piauí, Brésil) et d'autres sites pléistocènes du Brésil. Abréviations : L, longueur ; I, largeur; H, hauteur; D, droit ; G, gauche ; DT, diamètre transversal; DAP, diamètre antéro-postérieur; prox, proximal; dia, diaphyse; dist, distal; lat, latéral; med, médial; maxi, maximal; artic, articulaire; SRN, São Raimundo Nonato; ${ }^{*}$, dimension approximative.

\begin{tabular}{|c|c|c|c|c|c|c|}
\hline \multirow[b]{2}{*}{ Mc IV } & \multicolumn{2}{|c|}{$\begin{array}{c}\text { Toxodon platensis } \\
\text { (Serra Capivara) }\end{array}$} & \multicolumn{4}{|c|}{ Piauhytherium capivarae n. gen., n. sp. } \\
\hline & \multicolumn{2}{|c|}{$\begin{array}{c}\text { Antonião } \\
1655 \\
\text { G }\end{array}$} & $\begin{array}{c}\text { Porcos } \\
187847 \\
\text { D }\end{array}$ & $\begin{array}{c}\text { Porcos } \\
188050 \\
\text { G }\end{array}$ & $\begin{array}{c}\text { Currais Novos } \\
\text { G }\end{array}$ & $\begin{array}{c}\text { Taperoá (PB) } \\
3567 \text { V } \\
\text { D }\end{array}$ \\
\hline $\begin{array}{l}\text { Longueur } \\
\text { DT prox. } \\
\text { DAP prox. } \\
\text { DT dia. } \\
\text { DAP dia. } \\
\text { DT distal } \\
\text { DT artic. distal } \\
\text { DAP artic. Distal }\end{array}$ & \multicolumn{2}{|c|}{$\begin{array}{c}130 \\
60 \\
>56 \\
48 \\
42 \\
67,5 \\
58 \\
48\end{array}$} & $\begin{array}{l}113,5 \\
49^{\star} \\
53,5 \\
42,5 \\
30,5 \\
61,5 \\
51 \\
44 \\
\end{array}$ & $\begin{array}{c}119,5 \\
53,5 \\
62,5 \\
44 \\
37,5 \\
63,5 \\
56,5 \\
46 \\
\end{array}$ & $\begin{array}{l}120,5 \\
55 \\
57 \\
43,5 \\
37,5 \\
63 \\
53 \\
48,5\end{array}$ & $\begin{array}{c}121 \\
54 \\
47^{\star} \\
42,5 \\
36 \\
57,5 \\
50 \\
42,5 \\
\end{array}$ \\
\hline \multirow{2}{*}{ DAP artic. Distal } & \multicolumn{6}{|c|}{ T. platensis (Brésil) } \\
\hline & $\begin{array}{l}\text { Brésil } \\
\text { Museu Rio } \\
602 \mathrm{~V} \\
\text { D }\end{array}$ & $\begin{array}{l}\text { Laje Grande } \\
\text { (PE) } \\
\text { Museu Rio } \\
186 \mathrm{~V} \\
\text { D }\end{array}$ & $\begin{array}{c}\text { Laje Grande } \\
\text { (PE) } \\
\text { Museu Rio } \\
17 \text { V } \\
\text { D }\end{array}$ & $\begin{array}{c}\text { Laje Grande } \\
\text { (PE) } \\
\text { Museu Rio } \\
18 \text { V } \\
\text { G }\end{array}$ & $\begin{array}{l}\text { Laje Grande } \\
\text { (PE) } \\
\text { Museu Rio } \\
19 \text { V } \\
\text { G }\end{array}$ & $\begin{array}{c}\text { Laje Grande } \\
\text { (PE) } \\
\text { Museu Rio } \\
187 \mathrm{~V} \\
\text { G }\end{array}$ \\
\hline $\begin{array}{l}\text { Longueur } \\
\text { DT prox. } \\
\text { DAP prox. } \\
\text { DT dia. } \\
\text { DAP dia. } \\
\text { DT distal } \\
\text { DT artic. distal } \\
\text { DAP artic. Distal }\end{array}$ & $\begin{array}{l}121,5 \\
53 \\
51 \\
48 \\
34,5 \\
60 \\
52,5 \\
47 \\
\end{array}$ & $\begin{array}{l}122,5 \\
57 \\
66 \\
49,5 \\
41,5 \\
66 \\
57,5 \\
50,5 \\
\end{array}$ & $\begin{array}{c}134 \\
60 \\
69 \\
47 \\
42 \\
64,5 \\
55 \\
52 \\
\end{array}$ & $\begin{array}{l}130,5 \\
54 \\
61 \\
50 \\
41 \\
59 \\
57 \\
48 \\
\end{array}$ & $\begin{array}{l}48 \\
41,5 \\
65 \\
56 \\
52,5 \\
\end{array}$ & $\begin{array}{r}130 \\
55 \\
62 \\
46 \\
42 \\
56 \\
60 \\
54 \\
\end{array}$ \\
\hline \multirow{2}{*}{ DAP artic. Distal } & \multicolumn{6}{|c|}{ T. platensis (Argentine) } \\
\hline & & nombre & moyenne & $\operatorname{mini}$ & maxi & \\
\hline $\begin{array}{l}\text { Longueur } \\
\text { DT prox. } \\
\text { DAP prox. } \\
\text { DT dia. } \\
\text { DAP dia. } \\
\text { DT distal } \\
\text { DT artic. distal } \\
\text { DAP artic. Distal }\end{array}$ & & $\begin{array}{l}3 \\
2 \\
2 \\
2 \\
2 \\
2 \\
2 \\
2\end{array}$ & $\begin{array}{r}127,50 \\
62,00 \\
71,00 \\
52,00 \\
44,00 \\
68,50 \\
60,00 \\
52,25\end{array}$ & $\begin{array}{l}123 \\
60 \\
70 \\
51 \\
43,5 \\
67 \\
59 \\
49,5\end{array}$ & $\begin{array}{l}134 \\
64 \\
72 \\
53 \\
44,5 \\
70 \\
61 \\
55\end{array}$ & \\
\hline
\end{tabular}


TABLEAU 10. - Dimensions comparées des fémurs des Toxodontidae (Toxodon platensis Owen, 1837 et Piauhytherium capivarae n. gen., n. sp.) de la région du Parc national Serra da Capivara (SE du Piaúi, Brésil) et d'autres sites pléistocènes du Brésil. Abréviations : L, longueur; I, largeur; H, hauteur; D, droit ; G, gauche ; DT, diamètre transversal; DAP, diamètre antéro-postérieur; prox, proximal; dia, diaphyse; dist, distal; lat, latéral; med, médial; maxi, maximal; artic, articulaire; SRN, São Raimundo Nonato; *, dimension approximative.

\begin{tabular}{|c|c|c|c|c|c|c|c|c|c|c|c|c|c|c|c|}
\hline \multirow[b]{2}{*}{ Fémur } & \multicolumn{15}{|c|}{ Piauhytherium capivarae n. gen., n. sp. } \\
\hline & 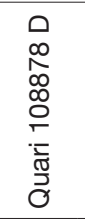 & 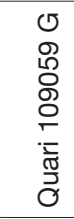 & 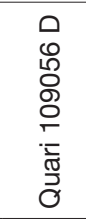 & 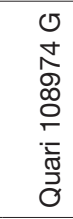 & 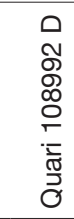 & 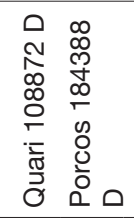 & $\begin{array}{l}\text { g } \\
\text { L } \\
\infty \\
\infty \\
0 \\
0 \\
0 \\
0 \\
0 \\
0 \\
0\end{array}$ & $\begin{array}{l}\overline{5} \\
\text { Lo } \\
\infty \\
\infty \\
0 \\
0 \\
0 \\
0 \\
0\end{array}$ & $\begin{array}{l}0 \\
0 \\
0 \\
0 \\
\infty \\
0 \\
0 \\
0 \\
0 \\
0\end{array}$ & $\begin{array}{l}\infty \\
\infty \\
\infty \\
\infty \\
\infty \\
\infty \\
0 \\
0 \\
0 \\
0\end{array}$ & 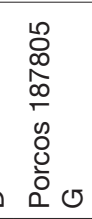 & $\begin{array}{l}\text { O } \\
0 \\
\infty \\
\infty \\
0 \\
0 \\
0 \\
0 \\
0 \\
0\end{array}$ & $\begin{array}{l}\widetilde{0} \\
0 \\
\stackrel{0}{0} \\
\infty \\
0 \\
0 \\
0 \\
0 \\
0\end{array}$ & 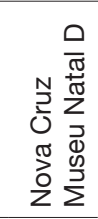 & 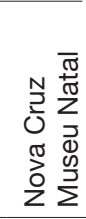 \\
\hline Longueur & 550 & 526 & 558 & 500 & & 490 & 516 & & 485 & 486 & 543 & 575 & & $>542$ & 575 \\
\hline DT pr & 216 & 208 & 207 & 200 & & $190 * 188$ & 195,5 & & 195,5 & 185 & 196 & 223 & & & 208 \\
\hline DT têt & 89,5 & & 92 & $80^{*}$ & & 82,5 & 93,5 & & 86 & 88,5 & 592,5 & 97,5 & & & 99 \\
\hline & 93 & 88 & 95 & & & $81 * 90$ & 91 & & 85 & 83 & 93,5 & 94 & & & 94, \\
\hline DT dis & 78 & 69 & 72 & 78 & & $78 \quad 71,5$ & 75,5 & & 72 & 71 & 69,5 & 71,5 & 73,5 & 78 & 76 \\
\hline & 55 & 59 & 58 & 49 & & $56 \quad 55,5$ & 53,5 & & 56 & 54,5 & 555 & 63 & 64,5 & & 66 \\
\hline & 145,5 & 145 & 147,5 & & 133 & 184 & 136,5 & 130,5 & 5135 & 134 & 140,5 & 5155 & 156,5 & 159 & 152,5 \\
\hline DAP distal & 174 & 170 & 174 & 158 & 154 & $148^{\star}$ & 161 & 162 & 149 & 146,5 & 5164 & 178,5 & 5180,5 & & 178,5 \\
\hline
\end{tabular}

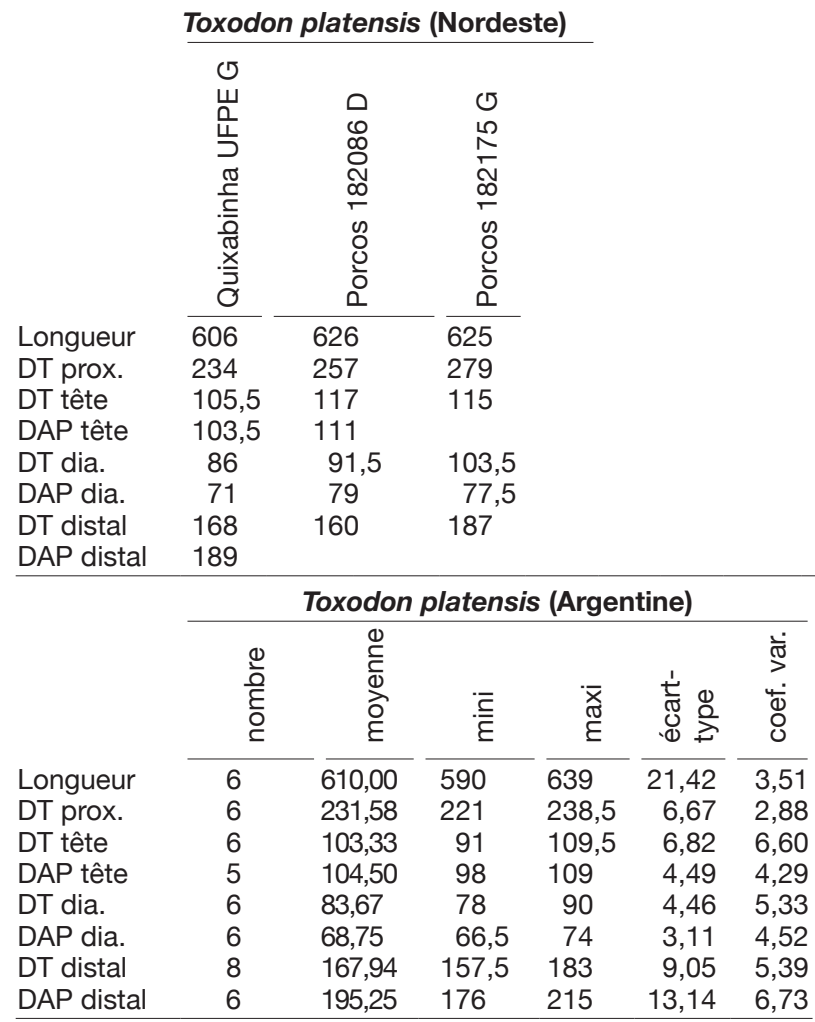


TABLEAU 11. - Dimensions comparées des tibias des Toxodontidae (Toxodon platensis Owen, 1837 et Piauhytherium capivarae n. gen., n. sp.) de la région du Parc national Serra da Capivara (SE du Piauí, Brésil) et d'autres sites pléistocènes du Brésil. Abréviations : L, longueur; I, largeur; H, hauteur; D, droit ; G, gauche ; DT, diamètre transversal; DAP, diamètre antéro-postérieur; prox, proximal; dia, diaphyse; dist, distal; lat, latéral; med, médial; maxi, maximal; artic, articulaire; SRN, São Raimundo Nonato; *, dimension approximative.

\begin{tabular}{|c|c|c|c|c|c|c|}
\hline \multirow[b]{2}{*}{ Tibia } & \multicolumn{3}{|c|}{ Piauhytherium capivarae n. gen., n. sp. } & & \multicolumn{2}{|c|}{ Toxodon platensis (Brésil) } \\
\hline & $\begin{array}{c}\text { Quari } \\
\text { FUMDHAM } \\
108967 \\
\text { D }\end{array}$ & $\begin{array}{c}\text { Quari } \\
\text { FUMDHAM } \\
\text { 79644-6 } \\
\text { D }\end{array}$ & $\begin{array}{c}\text { Porcos } \\
\text { FUMDHAM } \\
187920 \\
\text { D }\end{array}$ & & $\begin{array}{c}\text { Uruguaiana } \\
\text { Museu Rio } \\
\text { D }\end{array}$ & $\begin{array}{c}\text { Bom Jardim } \\
\text { Recife (PE) }\end{array}$ \\
\hline Longueur & 331 & & 320 & & 385 & \\
\hline DT prox. & 148 & & 123 & & 153 & \\
\hline DAP prox. & & & 140 & & 169 & \\
\hline DT dia. & 37 & & 37 & & 41,5 & \\
\hline DAP dia. & $79^{*}$ & & 76 & & 82,5 & \\
\hline DT distal & 95 & 90 & 81,5 & & 101 & 112 \\
\hline \multirow[t]{3}{*}{ DAP distal } & 86 & $78^{*}$ & 73 & & 89 & 73 \\
\hline & \multicolumn{6}{|c|}{ T. platensis (Argentine) } \\
\hline & nombre & moyenne & $\operatorname{mini}$ & maxi & écart-type & coef. var. \\
\hline Longueur & 5 & 366,80 & 352 & 385 & 12,48 & 3,40 \\
\hline DT prox. & 5 & 152,70 & 140 & 161,5 & 8,03 & 5,26 \\
\hline DAP prox. & 3 & 167,83 & 161 & 173,5 & 6,33 & 3,77 \\
\hline DT dia. & 5 & 44,50 & 38,5 & 53 & 6,03 & 13,55 \\
\hline DAP dia. & 5 & 84,80 & 79 & 90 & 4,28 & 5,05 \\
\hline DT distal & 4 & 100,13 & 96,5 & 103 & 2,72 & 2,72 \\
\hline DAP distal & 5 & 85,60 & 81 & 89 & 2,90 & 3,39 \\
\hline
\end{tabular}


TABLEAU 12. - Dimensions comparées des astragales de Toxodon platensis Owen, 1837 de divers sites pléistocènes du Brésil. Abréviations: L, longueur; I, largeur; H, hauteur; D, droit ; G, gauche ; DT, diamètre transversal; DAP, diamètre antéro-postérieur; prox, proximal; dia, diaphyse; dist, distal ; lat, latéral; med, médial ; maxi, maximal; artic, articulaire; SRN, São Raimundo Nonato; * signifie que la dimension qui précède est approximative.

\begin{tabular}{|c|c|c|c|c|c|}
\hline \multirow[b]{2}{*}{ Astragale } & \multicolumn{5}{|c|}{ T. platensis (Brésil) } \\
\hline & $\begin{array}{c}\text { Chuí (RS) } \\
\text { Museu Rio } \\
3110 \\
\text { D }\end{array}$ & $\begin{array}{c}\text { ? São Paulo } \\
\text { Museu Rio } \\
2386 \text { V } \\
\text { G }\end{array}$ & $\begin{array}{c}\text { Albardão (RS) } \\
\text { Museu Rio } \\
3110 \text { V } \\
\text { D }\end{array}$ & $\begin{array}{c}\text { Albardão (RS) } \\
\text { Museu Rio } \\
3111 \text { V } \\
\text { G }\end{array}$ & $\begin{array}{c}\text { Baraúna }(\mathrm{RN}) \\
\text { Museu Natal } \\
148 \mathrm{~V} \\
\mathrm{D}\end{array}$ \\
\hline DT maxi & 86 & 86 & 86 & 85 & 89 \\
\hline $\mathrm{H}$ maxi & 72,5 & 63 & 68,5 & 72,5 & 71 \\
\hline $\mathrm{H}$ lat. & 64 & 56 & 64,5 & 67,5 & 63,5 \\
\hline DAP lat. & & 30 & 37 & 35,5 & 33 \\
\hline H méd. & 70,5 & 61 & 69 & 74 & 67 \\
\hline DAP méd. & 57 & 53,5 & 57 & 69 & 59,5 \\
\hline DT partie dist. & & & & & 55,5 \\
\hline DAP partie dist. & & & & & 51 \\
\hline H milieu & 46 & 48 & 55 & 59 & 47,5 \\
\hline Dist. des 2 lèvres & 60 & 49 & & & 47 \\
\hline
\end{tabular}

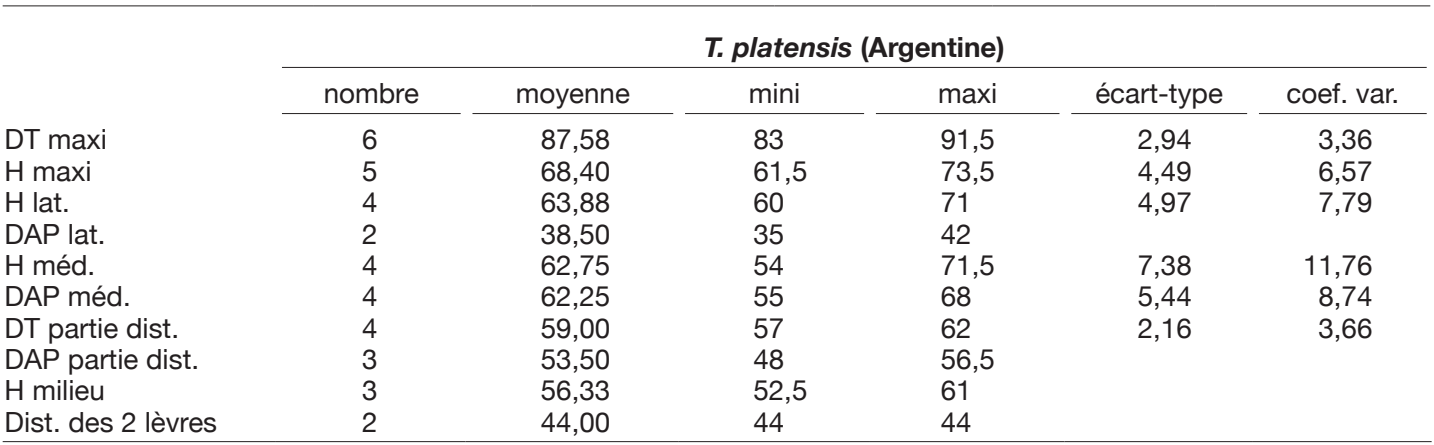


TABleAu 13. - Dimensions comparées des calcanéums des Toxodontidae (Toxodon platensis Owen, 1837 et Piauhytherium capivarae n. gen., n. sp.) de la région du Parc national Serra da Capivara (SE du Piauí, Brésil) et d'autres sites pléistocènes du Brésil. Abréviations : L, longueur; I, largeur; H, hauteur; D, droit ; G, gauche ; DT, diamètre transversal; DAP, diamètre antéro-postérieur; prox, proximal; dia, diaphyse; dist, distal; lat, latéral ; med, médial ; maxi, maximal ; artic, articulaire; SRN, São Raimundo Nonato; *, dimension approximative.

\begin{tabular}{|c|c|c|c|c|c|c|}
\hline \multirow[b]{2}{*}{ Calcanéum } & \multicolumn{2}{|c|}{ Toxodon platensis } & \multicolumn{2}{|c|}{ P. capivarae n. gen., n. sp. } & & \\
\hline & \multicolumn{2}{|c|}{$\begin{array}{c}\text { Garrincho } \\
\text { FUMDHAM } \\
37016 \\
\text { D }\end{array}$} & $\begin{array}{c}\text { Quari } \\
\text { FUMDHAM } \\
\text { 79803-8 } \\
\text { G }\end{array}$ & $\begin{array}{c}\text { São Vitor } \\
\text { FUMDHAM } \\
48912 \\
\text { D } \\
\end{array}$ & & \\
\hline Hauteur & \multicolumn{2}{|c|}{138} & 125 & & & \\
\hline DT tête & \multirow{2}{*}{\multicolumn{2}{|c|}{$\begin{array}{l}79 \\
60\end{array}$}} & 63 & & & \\
\hline DAP tête & & & & & & \\
\hline DT milieu & \multicolumn{2}{|c|}{$\begin{array}{r}62 \\
102\end{array}$} & 44 & 39,5 & & \\
\hline DT maxi & \multicolumn{2}{|c|}{102} & & 92 & & \\
\hline \multirow[t]{3}{*}{ DAP maxi } & & $4^{*}$ & 60 & 62 & & \\
\hline & \multicolumn{6}{|c|}{ T. platensis (Argentine) } \\
\hline & nombre & moyenne & mini & $\operatorname{maxi}$ & écart-type & coef. var. \\
\hline Hauteur & 13 & 132,27 & 123,5 & 141,5 & 5,17 & 3,91 \\
\hline DT tête & 12 & 71,13 & 63,5 & 78 & 4,51 & 6,34 \\
\hline DAP tête & 12 & 64,38 & 53 & 73,5 & 6,74 & 10,47 \\
\hline DT milieu & 8 & 59,13 & 54 & 70 & 5,54 & 9,37 \\
\hline DT maxi & 12 & 101,67 & 92 & 111 & 5,27 & 5,18 \\
\hline \multirow[t]{3}{*}{ DAP maxi } & 12 & 64,75 & 57 & 75 & 6,53 & 10,08 \\
\hline & \multicolumn{6}{|c|}{ T. platensis (Brésil) } \\
\hline & $\begin{array}{c}\text { Laje Grande } \\
\text { Museu Rio } \\
20 \mathrm{~V} \\
\text { D } \\
\end{array}$ & $\begin{array}{c}\text { Laje Grande } \\
\text { Museu Rio } \\
190 \text { V } \\
\text { G }\end{array}$ & $\begin{array}{c}\text { Laje Grande } \\
\text { Museu Rio } \\
22 \text { V } \\
\text { G }\end{array}$ & $\begin{array}{c}\text { ? São Paulo } \\
\text { Museu Rio } \\
2384 \mathrm{~V} \\
\text { G }\end{array}$ & $\begin{array}{l}\text { ? São Paulo } \\
\text { Museu Rio } \\
2384 \text { V }\end{array}$ & $\begin{array}{c}\text { Albardão (RS) } \\
\text { Museu Rio } \\
3112 \text { V } \\
\text { D }\end{array}$ \\
\hline Hauteur & 132 & 128 & 125 & 132 & 125,5 & 129 \\
\hline DT tête & 80,5 & 79,5 & 63 & 66 & 73 & 69 \\
\hline DAP tête & 60 & 55,5 & 49 & 58 & 62,5 & 53 \\
\hline DT milieu & & & & & & 57 \\
\hline DT maxi & $116,5^{\star}$ & & 87 & 98,5 & 105 & 92 \\
\hline DAP maxi & 60 & 57 & 51,5 & 64 & 61 & 58,5 \\
\hline
\end{tabular}


TABleAu 14. - Dimensions comparées des Métatarsiens II de Toxodon platensis Owen, 1837 de la région du Parc national Serra da Capivara (SE du Piauí, Brésil) et d'autres sites pléistocènes du Brésil. Abréviations : L, longueur; I, largeur; H, hauteur; D, droit ; G, gauche ; DT, diamètre transversal; DAP, diamètre antéro-postérieur; prox, proximal; dia, diaphyse; dist, distal; lat, latéral; med, médial ; maxi, maximal; artic, articulaire; SRN, São Raimundo Nonato; *, dimension approximative.

\begin{tabular}{|c|c|c|c|}
\hline \multirow[b]{2}{*}{ Mt II } & \multicolumn{3}{|c|}{ Toxodon platensis (Brésil) } \\
\hline & $\begin{array}{c}\text { Antonião } \\
\text { FUMDHAM } \\
1425 \text { G }\end{array}$ & $\begin{array}{c}\text { Taperoá } \\
\text { Museu Rio } \\
\text { G }\end{array}$ & $\begin{array}{c}\text { Chuí (RS) } \\
\text { Museu Rio } \\
3113 \text { V D }\end{array}$ \\
\hline Longueur & 89,5 & 84 & 84 \\
\hline DT prox. & 45 & 41 & 45 \\
\hline DAP prox. & 44 & 41 & 47 \\
\hline DT dia. & 35,5 & 38 & 37,5 \\
\hline DAP dia. & 33,5 & 36 & 35 \\
\hline DT distal & 45,5 & 47 & 48 \\
\hline DT artic. distal & 40 & 43,5 & 43,5 \\
\hline DAP artic. dist. & 41 & 43 & 46 \\
\hline
\end{tabular}

Toxodon platensis (Argentine)

\begin{tabular}{|c|c|c|c|c|c|c|}
\hline & \\
\hline & nombre & moyenne & $\operatorname{mini}$ & $\operatorname{maxi}$ & écart-type & coef. var. \\
\hline Longueur & 5 & 90,90 & 84 & 95,5 & 4,34 & 4,77 \\
\hline DT prox. & 4 & 46,63 & 44 & 51 & 3,09 & 6,63 \\
\hline DAP prox. & 5 & 49,60 & 47 & 52 & 2,07 & 4,18 \\
\hline DT dia. & 5 & 41,60 & 37,5 & 43,5 & 2,48 & 5,97 \\
\hline DAP dia. & 5 & 36,60 & 35 & 38 & 1,29 & 3,54 \\
\hline DT distal & 5 & 53,90 & 48 & 56,5 & 3,38 & 6,27 \\
\hline DT artic. distal & 5 & 45,30 & 43,5 & 47 & 1,30 & 2,88 \\
\hline DAP artic. dist. & 5 & 49,60 & 46 & 55,5 & 3,56 & 7,18 \\
\hline DAP artic. dist. & 5 & 49,60 & 46 & 55,5 & 3,56 & 7,18 \\
\hline
\end{tabular}

TABlEAU 15. - Dimensions comparées des Métatarsiens III de Toxodon platensis Owen, 1837 de la région du Parc national Serra da Capivara (SE du Piauí, Brésil). Abréviations : L, longueur; I, largeur; H, hauteur; D, droit ; G, gauche ; DT, diamètre transversal; DAP, diamètre antéro-postérieur; prox, proximal; dia, diaphyse; dist, distal; lat, latéral; med, médial; maxi, maximal; artic, articulaire; SRN, São Raimundo Nonato; *, dimension approximative.

\begin{tabular}{|c|c|c|c|c|}
\hline \multirow[b]{2}{*}{ Mt III } & \multicolumn{2}{|c|}{$\begin{array}{l}\text { Toxodon platensis } \\
\text { (Nordeste) }\end{array}$} & & \\
\hline & $\begin{array}{l}\text { Antonião } \\
\text { FUMDHAM } \\
\text { A } 5355 \text { G }\end{array}$ & $\begin{array}{c}\text { Garrincho } \\
\text { FUMDHAM } \\
47734 \text { G }\end{array}$ & & \\
\hline Longueur & 82 & 91 & & \\
\hline DT prox. & 54 & 58 & & \\
\hline DAP prox. & $>39$ & $48^{*}$ & & \\
\hline DT dia. & 51,5 & 53 & & \\
\hline DAP dia. & 31,5 & 32 & & \\
\hline DT distal & 61,5 & 68,5 & & \\
\hline DT artic. distal & 52 & 55 & & \\
\hline \multirow[t]{3}{*}{ DAP artic. dist. } & 43 & 48 & & \\
\hline & \multicolumn{4}{|c|}{ T. platensis (Argentine) } \\
\hline & nombre & moyenne & $\operatorname{mini}$ & maxi \\
\hline Longueur & 3 & 97,17 & 93 & 104 \\
\hline DT prox. & 3 & 56,17 & 54,5 & 59 \\
\hline DAP prox. & 2 & 49,50 & 43 & 56 \\
\hline DT dia. & 2 & 51,75 & 51,5 & 52 \\
\hline DAP dia. & 1 & 31,50 & & \\
\hline DT distal & 3 & 66,50 & 65 & 67,5 \\
\hline DT artic. distal & 3 & 55,67 & 53,5 & 59 \\
\hline DAP artic. dist. & 3 & 47,83 & 47 & 48,5 \\
\hline
\end{tabular}

